Pacific Journal of Mathematics

TOPICS IN HARMONIC ANALYSIS ON SOLVABLE
ALGEBRAIC GROUPS 


\title{
TOPICS IN HARMONIC ANALYSIS ON SOLVABLE ALGEBRAIC GROUPS
}

ROGER E. Howe

\begin{abstract}
This paper consists of two parts, and in the first of these we develop the representation theory of solvable algebraic groups over a local field of characteristic zero in analogy with the work of Auslander and Kostant for solvable Lie groups. We show how all the representations arise and show that the Kirillov method of orbits applies to this situation. We find that the theory carries over completely and we discuss traces, CCR representations and we give a version of the Kostant independence of polarization theorem.

In the second part we take up the problems of decomposing the space of square integrable functions on a solvable Lie group modulo a discrete cocompact subgroup. We show how to reduce this problem to the special case when the nilradical of the solvable group is Heisenberg. These two sections represent the initial part of a comprehensive program in this direction to be completed later.
\end{abstract}

Introduction. The theory of harmonic analysis on nilpotent Lie groups was established essentially in one blow by A. A. Kirillov [21] in the early 1960's, after some initial researches by Dixmier. Subsequently, other authors ([16], [17], [24], [29], [37]) explored further topics, such as character theory, extension of these results to unipotent $p$-adic groups and more general nilpotent groups, and the spectral decomposition of arithmetic and adelic quotient spaces of unipotent groups. The methods initiated by Kirillov have been extraordinarily supple, and while harmonic analysis on nilpotent groups is hardly complete, I feel it may rightly be considered a mature subject.

Meanwhile, an effort was made to extend Kirillov's analysis to solvable Lie groups. Although the same philosophy eventually succeeded, considerably more sophisticated concepts were required. Several major attacks ([5], [6], [10]) yielded partial progress, but the essence of the problem was not exposed until 1968 with the announcement of Auslander-Kostant [3], and their results, with proofs, have only been published very recently [4]. The Auslander-Kostant results provide a complete description of the representations of a connected, simply connected type I solvable Lie group, and characterize which connected, simply connected solvable Lie groups are type I. More recently, L. Pukanszky [30] has very successfully attacked the problem of doing harmonic analysis on a general, nontype I, solvable Lie 
group. Thus it may be safely said that the basic phenomena of harmonic analysis on solvable Lie groups are understood, and the foundational era of this subject is over. However, very few of the more baroque developments, such as described above for nilpotent groups, have been considered. The present paper attempts a partial remedy for this grievous lack.

The paper consists of two parts, the first two of a projected five. The focus of the full project is harmonic analysis on algebraic solvable groups over algebraic number fields. The fifth part would consist of miscellany concerning the harmonic analysis of the rational points of such a group, or of arithmetic subgroups, considered as abstract discrete groups. The first four parts are coordinated and culminate in part four, which would treat adele groups. Thus the first three parts are in some sense preliminary to part four. Part one treats representations of $p$-adic algebraic groups. Parts two and three consider the problem of describing the spectrum of $L^{2}(\mathscr{S} / \Gamma)$ where $\mathscr{S}$ is a solvable Lie group, and $\Gamma$ a discrete subgroup such that $\mathscr{S} / \Gamma$ is compact. As seemingly always in this subject, the crucial case is that when the nilradical of $\mathscr{S}$ is a Heisenberg group. Part 2, presented here, is concerned with reduction to this case and is probably quite tedious. Part 3 would look at the multiplicity theory of abelian extensions of Heisenberg groups. This part of the theory is in my opinion very pretty, and provides relief after part 2. The situation is very concrete, and the results connect with the Weil representation [36] on one hand, with the conjecture of Langlands [22] on another, and with the recent announcement of AuslanderBrezin [1] on another.

We will now describe in more detail the content of parts one and two.

In the first part, we develop the representation theory of characteristic zero $p$-adic solvable algebraic groups in analogy with the work of Auslander-Kostant [4] on real groups. We show how all the representations arise, and show that the method of orbits ("Kirillov theory") applies completely to an open normal subgroup containing the unipotent radical $\mathscr{N}$ of an arbitrary $p$-adic solvable algebraic group $\mathscr{S}$. We show that any irreducible unitary representation of $\mathscr{S}$ can be induced from a finite dimensional representation of some subgroup. We show that the Mackey obstruction for extending a representation from $\mathscr{N}$ to its isotropy group in $\mathscr{S}$ always vanishes. We show that the restriction of any representation of $\mathscr{S}$ to $\mathscr{N}$ is multiplicity free, and that if any two representations of $\mathscr{S}$ have the same restriction to $\mathscr{N}$, then one is the tensor product of a linear character of $\mathscr{S}$ with the other. We discuss traces, and characterize the CCR representations. All the above results are valid whether 
or not $\mathscr{S}$ is split. In addition, we give what we feel deserves to be considered a canonical parameterization for the entire dual of $\mathscr{S}$, for $\mathscr{S}$ split, by proving an analogue of Kostant's “independence of polarization" theorem.

We obtain our results with much less effort than Auslander-Kostant expend for theirs. There are two main reasons for this, I feel. First, we restrict our attention to algebraic groups. Besides being structurally simpler than general solvable groups, algebraic groups are all type I, so no questions of type enter. Secondly, $p$-adic solvable groups have very large open compact subgroups to which Kirillov theory applies perfectly. This, in particular, allows one to induce from nonalgebraic subgroups, providing a much simpler analogue of Kostant's scheme of complex polarizations. Together, these simplifications facilitate the analysis tremendously. However, we must work harder to parametrize the representations, and for less complete results. It is difficult to say at this point whether this limitation is fundamental or due to lack of insight. It is possible, though, that it is in the nature of things. The Auslander-Kostant analysis, as beautiful as it is, may be special to real groups, since only over the reals can an exponential map be globally defined, permitting a blurring of the distinction between $\boldsymbol{R}$ and $\boldsymbol{R}^{x}$. Thus, in our retreat from a complete "orbit picture" we have accepted the additive and multiplicative groups, the unipotent and semisimple, as co-equal and fundamental, and have constructed the full theory using both of them. Thus solvable groups provide, as they should, a transition from unipotent groups, where Kirillov theory and additive principles dominate, to semisimple groups, where Kirillov theory is much less powerful, and multiplicative features prevail; and they lend thereby a coherence and unity to the whole theory of representations of algebraic groups.

In $\S 2$, we take up the problem of decomposing $L^{2}(\mathscr{S} \mid \Gamma)$, where $\mathscr{S}$ is solvable Lie group and $\Gamma$ is a discrete subgroup, such that $\mathscr{S} / \Gamma$ is compact. This problem was solved in [16] and [34] for the case of $\mathscr{S}$ nilpotent. The section begins with an elementary discussion on the general features of the problem, and tries to show how the methods involved in the solution of the problem for nilpotent Lie groups generalize partially, and to pinpoint the special structural features of nilpotent Lie groups that permit the methods to work completely. It is pointed out that an arbitrary locally compact separable metabelian group is susceptible to the same methods.

We then focus on solvable Lie groups. Let $\mathscr{S}$ and $\Gamma$ be as above and let $\mathscr{N}$ be the nilradical of the connected component of $\mathscr{S}$. We assume $\mathscr{S} / \mathscr{N}$ is abelian. The main result is a reduction 
of the problem to the case when $\mathscr{N}$ is Heisenberg and $\mathscr{S} \cong \mathscr{T} \mathrm{X}_{S}$ $\mathscr{N}$ (semidirect product) where $\mathscr{T}$ is abelian, acts effectively on $\mathscr{N}$ but trivially on the center of $\mathscr{N}$ and $\mathscr{T} / \mathscr{T} \cap \Gamma$ is compact. This reduction is based on the known solution for nilpotent groups and is given in several steps. After several preliminary reductions, it is shown (Theorem 2.1) how to compute the multiplicity in $L^{2}(\mathscr{S} / \Gamma)$ of a representation $\rho$ of $\mathscr{S}$ induced from a representation $\tau$ of a certain type of subgroup $\mathscr{R}$ of $\mathscr{S}$, in terms of the multiplicity of $\tau$ in $L^{2}(\mathscr{R} / \mathscr{R} \cap \Gamma)$. Then a structural result (Propositions 2.3 and 2.4) shows that the problem for $\mathscr{R}$ is essentially the problem for $\mathscr{S}$ of the stated special form.

Theorem 2.1 is a clear analogue of a theorem on finite groups, and is developed in the spirit of analogy. At a critical point (Lemma 2.4 ) in the proof, we use the structure of $\mathscr{N}$ as an unipotent algebraic group defined over $Q$ to prove our construction provides enough representations to fill up $L^{2}(\mathscr{S} \mid \Gamma)$. It is precisely this step which allows us to remedy the deficiency noted in the preliminary discussion at the beginning of the section and obtain the full desired result for our case.

I. The local story. In this section $F$ will denote a $p$-adic field of characteristic zero - that is, a finite extension of $\boldsymbol{Q}_{p}$, the $p$-adic numbers. For the purposes of argument in this section, we will assume $p$ is odd, in order not to have the extra complications that arise when $p=2$. The results all remain valid with perhaps slight changes for $p=2$. The modifications necessary in the arguments are technical and time-consuming, and will not be given.

$\mathscr{S}$ will denote a connected affine solvable algebraic group defined over $F$. $\mathscr{S}_{F}$ will denote the $F$-rational points of $\mathscr{S}$. We may write $\mathscr{S}=\mathscr{T} \times_{s} \mathscr{N}$ (semidirect product), where $\mathscr{T}$ is a maximal torus of $\mathscr{S}$ defined over $F$, and $\mathscr{N}$ is the unipotent radical of $\mathscr{L}$. Then also $\mathscr{S}_{F}=\mathscr{T}_{F} X_{S} \mathscr{N}_{F}$. See [8].

Note. We will commit reasonably often the barbarism of referring to the $F$-rational points of an algebraic group over $F$ simply as an algebraic group.

We suppose $\mathscr{S} \subseteq \underline{\mathrm{Gl}}_{n}$ for some $n$. Then $\mathscr{S}_{F} \subseteq \mathrm{Gl}_{n}(F)$ as a (Zariski) closed subgroup, so $\mathscr{S}_{F}$ inherits a locally compact topology. Let $\underline{\underline{S}} \underline{\underline{T}}$, and $\underline{\underline{N}}$ be the Lie algebras of $\underline{\mathscr{S}}, \underline{\mathscr{T}}$, and $\underline{\underline{N}}$. Then $S_{F}, T_{F}$, and $N_{F}$ may be regarded as subspaces of $M_{n}(F)$, the $n \times n$ matrices with entries in $F . \quad M_{n}(F)$ may, of course, be regarded as the Lie algebra of $\mathrm{Gl}_{n}\left(F^{\prime}\right)$. We have $S_{F}=T_{F} \oplus N_{F}$ (direct sum). For any group $G$ 
mentioned above, let $\operatorname{Ad} G$ denote the adjoint action of $G$ on its Lie algebra.

As is well-known, one may define on a suitably small neighborhood $U$ of the origin in $M_{n}(F)$, the exponential map exp: $U \rightarrow \mathrm{Gl}_{n}(F)$ by the usual formula. The map exp will be a homeomorphism onto its image, which will be a neighborhood of the identity in $\mathrm{Gl}_{n}(F)$. The inverse mapping, from $\exp U$ to $U$, will be denoted log. Haar measure on $U$ is sent to Haar measure on $\exp U$ by exp. $U$ may be chosen to be invariant under $\mathrm{Ad} \mathrm{Gl}_{n}(F)$. Then $U$ will contain all nilpotent elements in $M_{n}(F)$, and in particular $N_{F} \subseteq U$.

Let $R$ be the ring of integers in $F$, and let $\pi$ be a prime element of $R$. Let $V \subseteq S_{F}$ be an open compact $R$-module in $S_{F}$. Set $[V, V]=$ $\left\{\sum_{i}\left[v_{i}, w_{i}\right], v_{i}, w_{i} \in V\right\}$, where [ $]$ denotes the Lie bracket operation on $S_{F}$. We recall from [18], that $V$ is called e.e. if $[V, V] \subseteq \pi^{\beta} V$, where $\beta$ is a suitable power of $\pi$. We refer to [18] for the precise definition of $\beta$. If $V$ is e.e., and $V \subseteq U$, then $C=\exp V$ will be a compact open subgroup of $\mathscr{S}_{F} . C$ is also said to be e.e. We have the following simple lemma.

Lemma 1.1. There is a compact open $R$-submodule $W$ of $T_{F}$, and a sequence of open compact $R$-submodules $\left\{Y_{i}\right\}_{i=1}^{\infty}$ of $N_{F}$, such that $Y_{i} \subseteq Y_{i+1}$, and $V_{i}=W \oplus Y_{i}$ is e.e. and contained in $U$, and $\bigcup_{i} Y_{i}=$ $N_{F}$. Then $C_{i}=\exp \left(V_{i}\right)$ are open compact e.e. subgroups of $\mathscr{S}_{F}$, and if $C_{0}=\exp W$, then $C_{i} \subseteq C_{i+1}$, and $C_{\infty}=\bigcup_{i} C_{i}=C_{0} \mathbf{X}_{S} \mathscr{N}_{F}$ is an open normal subgroup of $\mathscr{S}_{F}$.

Proof. Let ad $\mathrm{ad}_{T}$ be the adjoint operation of $T_{F}$ on $S_{F}$. Let $\check{\varkappa}^{i}\left(N_{F}\right)$ be the groups of the ascending central series of $N_{F}$. Then $\operatorname{ad}_{T}$ acts semi-simply on $N_{F}$ and preserves each $\check{\varkappa}^{i}\left(N_{F}\right)$. Let $N_{F}=\bigoplus_{j=1}^{m} B_{j}$ be a decomposition of $N_{F}$ into irreducible subspaces under $\operatorname{ad}_{T}$ such that for each $i$, there is $m_{i} \leqq m$ such that $\mathscr{\Sigma}^{i}\left(N_{F}\right)=\bigoplus_{j=1}^{m_{i}} B_{j}$. Then if we choose a sufficiently small neighborhood $W$ of zero in $T_{F}$, for any $t \in W, \operatorname{ad}_{T}(t)$ will have eigenvalues so small that we may choose open compact $R$-modules $Z_{j} \subseteq B_{j}$, such that $\operatorname{ad}_{T}(t)\left(Z_{j}\right) \subseteq \pi^{\beta} Z_{j}$. We may as well assume $W$ is also an $R$-module. Since $\left[N_{F}, \mathscr{Z}^{i}\left(N_{F}\right)\right] \subseteq$ $\mathscr{C}^{i+1}\left(N_{F}\right)$, and since if $X \subseteq N_{F}$ is compact, then $[X, X]$ is compact, we see that given any integer $l_{m}$, we may inductively, starting with $m$ and going down, choose $\left\{l_{j}\right\}_{j=m}^{1}$ such that $l_{j-1}>l_{j}$, and such that if $Y=\sum_{j=1}^{m} \pi^{-l_{j}} B_{j}$, then $T+Y$ is e.e. Then inductively choosing a sequence of $l_{m}$ 's larger and larger, we get a sequence $\left\{Y_{k}\right\}_{k=1}^{\infty}$ of open compact $R$-submodules of $N_{F}$ such that $Y_{i} \subseteq Y_{i+1}, V_{i}=W \oplus Y_{i}$ is e.e., and $\mathrm{U}_{i} Y_{i}=N_{F}$. 
If we have chosen $W$ small enough, then $W \subseteq U$. Since $U$ is open and invariant by $\operatorname{Ad~Gl}_{n}(F)$, whether or not $y \in M_{n}(F)$ is in $U$ is determined by the eigenvalues of $y$. But if $t \in T_{F}, n \in N_{F}$, then $t+n$ and $t$ have the same eigenvalues, since the action of $S_{F}$ on $F^{n}$ may be triangularized over some extension of $F$, by Lie's theorem. Hence $W \oplus Y_{i} \subseteq U$ for all $i$, and the lemma is proved.

Using Lemma 1.1, we may apply the result of [18] to $C_{0} \mathscr{N}_{F}$. For a locally compact group $G$, let $\hat{G}$ denote the primitive ideal space of $G . \quad \hat{G}$ is understood to have its standard hull-kernel topology (see [13]). For a type I group $\hat{G}$ may be regarded as the set of (equivalence classes of) irreducible unitary representations of $G$, suitably topologized. For such groups we will consider either a primitive ideal or a unitary representation as being a point of $\hat{G}$. For $G$ abelian, $\hat{G}$ is the familiar Pontryagin dual group.

Now $\bigcup_{i} V_{i}=W \oplus N_{F}=V_{\infty}$ is open in $S_{F}$, and exp: $V_{\infty} \rightarrow C_{0} \times_{S} \mathscr{N}_{F}=C_{\infty}$ is a homeomorphism and sends Haar measure to Haar measure. Ad $\mathscr{S}_{F}$ acting on $S_{F}$ preserves $V_{\infty}$ and we denote by Ad $C_{\infty}$ the action of $C_{\infty}$ on $V_{\infty}$ by Ad. Since $\operatorname{Ad} C_{\infty}$ is an action by automorphisms, by dualizing it we get an action $\mathrm{Ad}^{*} C_{\infty}$ of $C_{\infty}$ on $\hat{V}_{\infty}$, the Pontryagin dual of $V_{\infty}$. We denote the space of orbits for this action by $\hat{V}_{\infty} / \mathrm{Ad}^{*} C_{\infty}$. We give this space the quotient topology.

For a totally disconnected locally compact group $G$, let $C_{c}^{\infty}(G)$ be the Schwartz-Bruhat space of locally constant complex-valued functions of compact support. If $G$ is abelian, and $\hat{G}$ is also totally disconnected, then Fourier transform defines an isomorphism between $C_{c}^{\infty}(G)$ and $C_{c}^{\infty}(\hat{G})$. If $C \in C_{c}^{\infty}(G)$, let $\hat{f}$ denote its Fourier transform. A linear functional on $C_{c}^{\infty}(G)$ is called a distribution on $G$. The spaces of distributions on $G$ and $\hat{G}$ are also isomorphic by Fourier transform. $C_{c}^{\infty}(G)$ is, of course, an algebra under convolution. We define an involution, *, of $C_{c}^{\infty}(G)$ by $f^{*}(g)=\bar{f}\left(g^{-1}\right)$, for $g \in G$, where - denotes complex conjugate. Also $f * h$ is the convolution of $f$ and $h . A$ distribution $\theta$ on $G$ is positive-definite if $\theta(f * f) \geqq 0$ for all $f \in C_{c}^{\infty}(G)$.

All three groups, $C_{\infty}, V_{\infty}$, and $\hat{V}_{\infty}$, are totally disconnected. The exponential map defines an isomorphism from $C_{c}^{\infty}\left(C_{\infty}\right)$ to $C_{c}^{\infty}\left(V_{\infty}\right)$. Also, for $\psi \in \hat{V}_{\infty}$, we may regard $\psi$ as a function on $C_{\infty}$ by composing $\psi$ with $\log$.

It follows by simple arguments (see [31]) that all $\mathrm{Ad}^{*} \mathscr{N}_{F}$ orbits in $\hat{N}_{F}$ are closed. Since $C_{\infty} / \mathscr{N}_{F}$ is compact, it is very easy to see further that all $\mathrm{Ad}^{*} C_{\infty}$ orbits in $\hat{V}_{\infty}$ are closed. Now by using Theorem 1.1 of [18] and taking an inductive limit over the $C_{i}$ 's, one arrives at the following result. (To be absolutely certain of the applicability of Theorem 1.1, we may assume we have actually constructed $V_{i}$ so that $\left[V_{i}, V_{i}\right] \subseteq \pi^{\beta+1} V_{i}$.) 
Proposition 1.1. Notations as above.

(i) There is natural homeomorphism $\beta: \hat{V}_{\infty} / \mathrm{Ad}^{*} C_{\infty} \rightarrow \hat{C}_{\infty}$.

(ii) $C_{\infty}$ is C.C.R. (see [5]). Any irreducible representation $\rho$ of $C_{\infty}$ sends $C_{c}^{\infty}\left(C_{\infty}\right)$ to finite rank operators ( $\rho$ is "admissible"). In particular the distribution $\theta_{\rho}: f \rightarrow \operatorname{trace}(\rho(f))$ is defined. $\theta_{\rho}$ is a positive-definite distribution, called the character of $\rho$.

(iii) If $\mathscr{O} \cong \hat{V}_{\infty}$ is an $\mathrm{Ad}^{*} C_{\infty}$ orbit, then $\mathcal{O}$ carries a unique invariant measure, do, up to scalar multiples. Let $\rho=\beta(\mathcal{O})$. Then if do is properly normalized, $\theta_{\rho}(f)=\int_{0} \hat{f}_{1}$ do, where $f_{1}=f \circ \exp \epsilon$ $C_{c}^{\infty}\left(V_{\infty}\right)$.

(iv) (Bochner theorem) Any positive-definite invariant distribution on $C_{\infty}$ is the image under exp of a distribution on $V_{\infty}$, which $i s$ the Fourier transform of a positive $\mathrm{Ad}^{*} C_{\infty}$-invariant measure on $\hat{V}_{\infty}$.

(v) (Plancherel theorem) The $\delta$-distribution $(\delta(f)=f(1), 1=$ identity of $C_{\infty}$ ) is the Fourier transform of Haar measure on $\hat{V}_{\infty}$.

Notice that the above statement, in contrast to others of its ilk, says nothing about the concrete realization of representations. We now attend to this matter. We establish two results, one for $C_{\infty}$, the other for all of $\mathscr{S}_{F}$. The first one is analogous to Pukansky's condition ([32]) for polarizations on exponentiable solvable Lie groups to give an irreducible representation.

If $H \cong C_{\infty}$ is any subgroup, we say $H$ is e.e. if $\log H$ is an $R$ module and $[\log H, \log H] \subseteq \pi^{\beta} \log H$. Note that Proposition 1.1 will also apply to $H$. If $\psi \in \widehat{V}_{\infty}$, and $H \subseteq C_{\infty}$ is an e.e. subgroup, we will say $H$ is subordinate to $\psi$ if the restriction of $\psi \circ \log$ to $H$ is a linear character of $H$.

Proposition 1.2. Take $\psi \in \hat{V}_{\infty}$, and $H \subseteq C_{\infty}$ an e.e. subgroup subordinate to $\psi$. Suppose $H$ contains the isotropy group of $\psi$ under $\operatorname{Ad}^{*} C_{\infty}$. Let $\psi^{\prime} \in \hat{H}$ be the restriction of $\psi \circ \log$ to $H$. Let $\operatorname{ind}_{H}^{C_{\infty}} \psi^{\prime}$ denote the representation of $C_{\infty}$ induced from $\psi^{\prime}$ on $H$. Then a necessary and sufficient condition that ind $\mathrm{d}_{H}^{C_{\infty}} \psi^{\prime}$ be irreducible and hence equal to $\left.\beta\left(\mathrm{Ad}^{*} C_{\infty}(\psi)\right)\right)$ is that $\mathrm{Ad}^{*} H(\psi)=\psi+(\log H)^{\perp}$, where $(\log H)^{\perp}$ is the subgroup of $\hat{V}_{\infty}$ consisting of characters trivial on $\log H$-that is, the annihilator in $\hat{V}_{\infty}$ of log $H$.

REMARK. If we specialize the above lemma to the case when $\mathscr{S}$ is actually unipotent (in which case the proof simplifies drastically), we see that the result then transfers to the context of [17], giving a complement to Proposition 12 and preceding remarks. Also note that $H$ is not required to be an algebraic subgroup. Thus we allow for instance for inducing a representation of a Heisenberg group 
over a local field from an open subgroup which is compact modulo the center (of the Heisenberg group). See Lemma 1.2.

Proof. We begin by investigating the structure of $H$. Put $J=$ $\log H \subseteq V_{\infty} \subseteq S_{F}$. Let $J^{0}$ be the linear subspace of $S_{F}$ spanned by $J$. Then it is easy to see that $J^{0}$ is a Lie subalgebra of $S_{F}$, and that $J \subseteq J^{0}$ is open. Let $J^{1}=\bigcap_{x \in F} x J$. Then $J^{1} \cong J^{0}$ is a linear subspace, and may be described as the largest linear subspace of $J^{0}$ contained in $J$. Since $J$ is open in $J^{0}$, and is closed under [,$], J^{1}$ is actually an ideal in $J^{0} . J / J^{1}$ is compact.

Let $M$ be the isotropy group of $\psi$ under $\operatorname{Ad}^{*} C_{\infty}$. Let $A=\log M$. Then as in [18], it follows that $A=\left\{a \in V_{\infty}: \psi\left(\left[\alpha, V_{\infty}\right]\right)=1\right\}$, and $A$ is an e.e. $R$-submodule of $V_{\infty}$. By our assumptions $A \subseteq J$. Put $A^{0}=$ span of $A$ in $S_{F}$, and $A^{1}=$ largest linear subspace of $S_{F}$ contained in $A$. Then, just as above, $A^{1}$ and $A^{0}$ are subalgebras of $S_{F}, A^{1}$ is an ideal in $A^{0}, A / A^{1}$ is compact, and $A$ is open in $A^{0}$. Also $A^{0} \subseteq J^{0}$, and $A^{1} \subseteq J^{1} \subseteq N_{F}$.

Put $B^{0}=\left\{b \in S_{F}, \psi\left(\left[b, N_{F}\right]\right)=1\right\}$. Then $B^{0}$ is a linear subspace of $S_{F}$, and by the Jacobi identity, since $N_{F}$ is an ideal in $S_{F}, B^{0}$ is a subalgebra. If $b \in B^{0} \cap V_{\infty}$, then $\exp b$ exists, and a term by term development of $\operatorname{Ad} \exp b(n)$, for $n \in N_{F}$, shows $\operatorname{Ad}^{*} \exp b(\psi)_{\mid N_{F}}=\psi_{\left.\right|_{N_{F}}}$. Here $\chi_{\mid N_{F}}$ indicates the restriction of $\psi$ to $N_{F}$. Conversely if $c \in C_{\infty}$, and $\operatorname{Ad}^{*} c(\psi)_{\left.\right|_{F}}=\psi_{\left.\right|_{F}}$, then the inverse formula

$$
[\log c, n]=\sum_{j=1}^{\infty}(-1)^{j-1} j^{-1}(\operatorname{Ad}(c)-1)^{j}(n)
$$

for $n \in N_{F}$, shows that $\log c \in B^{0} \cap V_{\infty}$. Hence putting

$$
M^{0}=\exp \left(B^{0} \cap V_{\infty}\right),
$$

we conclude that $M^{0}$ is the isotropy group under $\mathrm{Ad}^{*} C_{\infty}$ of the restriction of $\psi$ to $N_{F}$.

On the other hand, the kernel of the restriction map from $\hat{V}_{\infty}$ to $\hat{N}_{F}$ is discrete, since $V_{\infty} / N_{F}$ is compact. Therefore, $M$, the isotropy group of $\psi$ under $\mathrm{Ad}^{*} C_{\infty}$ must contain an open neighborhood of the identity in $M^{0}$. Hence $A$ contains a neighborhood of zero in $B^{0}$. Since clearly $M \subseteq M^{0}$, we have $A^{0}=B^{0}$, so $B^{0} \subseteq J^{0}$. Thus, if $H^{0}=$ $\exp \left(J^{0} \cap V_{\infty}\right), M^{0} \cong H^{0}$.

Now we will show ind ${ }_{H}^{C_{\infty}} \psi^{\prime}$ is irreducible. We begin by showing $\rho^{0}=\operatorname{ind}_{H}^{H^{0}} \psi^{\prime}$ is irreducible. Put $H^{1}=\exp J^{1}$. Then $H$ is open in $H^{0}$, and $H^{1}$ is normal in $H^{0}$, and $H / H^{1}$ is compact. By reasoning very similar to that of Lemma 1.1, we can find e.e. subgroups $\left\{H_{i}\right\}_{i=0}^{\infty}$ such that $H=H_{0} \subseteq H_{i} \subseteq H_{i+1} \subseteq H^{0}, H^{0}=\bigcup_{i=0}^{\infty} H_{i}$, and with $H_{i}$ normal in $H_{i+1}$. Let $\rho_{i}=\operatorname{ind}_{H}^{H^{i}} \psi^{\prime}$. Assuming $\rho_{i}$ is irreducible, we will show $\rho_{i+1}$ is irreducible. Then in the limit $\rho^{0}$ will be irreducible. 
Let us recall the basic facts about inducing from and restricting to normal subgroups. If $G$ is a separable locally compact group, and $\alpha$ is an automorphism of $G$, then $\alpha$ acts on the representations of $G$ as follows. If $\sigma$ is a representation of $G$, then $\alpha(\sigma)(g)=\sigma\left(\alpha^{-1}(g)\right)$. This action may then be transferred to $\hat{G}$, the primitive ideal space of $G$. The resulting transformation is a homeomorphism of $\hat{G}$. Now if $G \cong G^{\prime}$ is a closed normal subgroup for some separable locally compact group $G^{\prime}$, then conjugation in $G^{\prime}$ results in an action of $G^{\prime}$ on $G$ by automorphisms, and thereby in an action of $G^{\prime}$ on $\hat{G}$. We write this action as $\mathrm{Ad}^{*} G^{\prime}$. Of course, under $\mathrm{Ad}^{*} G^{\prime}, G$ acts trivially. If $G$ is a type I group, and $\rho$ is an irreducible representation of $G$ (or if $G$ is not type I, but $\rho$ is C.C.R.), then $\operatorname{ind}_{G}^{G^{\prime}} \rho$ is irreducible if and only if the orbit under $\mathrm{Ad}^{*} G^{\prime} / G$ of the point in $\hat{G}$ corresponding to $\rho$ is a principal $G^{\prime} / G$ orbit; in other words, if and only if $g^{\prime} \in G^{\prime}$, and $\operatorname{Ad}^{*} g^{\prime}(\rho)=\rho$ implies $g^{\prime} \in G$. Whether or not $\operatorname{ind}_{G}^{G^{\prime}} \rho$ is irreducible the restriction of any subrepresentation of it to $G$ consists of a direct integral over all $\operatorname{Ad}^{*} G^{\prime}(\rho)$. See [5], [23] for details.

Returning now to our solvable group, note that $\rho_{i+1}=\operatorname{ind}_{H_{i}}^{H i+1} \rho_{i}$ by transitivity of induction. Suppose $\mathrm{Ad}^{*} h\left(\rho_{i}\right)=\rho_{i}$ for some $h \in H_{i+1}$. Let $J_{i}=\log H_{i}$. Since $H_{i}$ is e.e., Proposition 1.1 applies to it also. Let $\mathscr{O} \subseteq \widehat{J}_{i}$ be the $\mathrm{Ad}^{*} H_{i}$ orbit corresponding to $\rho_{i}$. Then since $\rho_{i}$ is induced from $\psi^{\prime}$, Proposition 1.3 of [18] shows $\mathcal{O}$ contains the restriction $\psi_{i}$ of $\psi$ to $J_{i}$. Now the naturality of the correspondence between $\hat{J}_{i} / \mathrm{Ad}^{*} H_{i}$ and $\hat{H}_{i}$ implies $\operatorname{Ad}^{*} h(\mathcal{O})=\mathscr{O}$. (This compatibility justifies the ubiquitous use of the $\mathrm{Ad}, \mathrm{Ad}^{*}$ notation.) Since $\mathcal{O}=\mathrm{Ad}^{*} H_{i}\left(\psi_{i}\right)$ we may assume, after altering $h$ by an element of $H_{i}$ if necessary, that $\mathrm{Ad}^{*} h\left(\psi_{i}\right)=\psi_{i}$. This in turn implies that $\operatorname{Ad}^{*} h(\psi) \in \psi+J_{i}^{\perp} \subseteq \psi+J_{0}^{\perp}$. Hence, modifying $h$ again, this time by an element of $H_{0}=H$, we will have $\operatorname{Ad}^{*} h(\psi)=\psi$. But then $h \in H \subseteq H_{i}$ by our assumption that $H$ contains the isotropy group of $\psi$. Hence $\rho_{i+1}$ is irreducible, and so $\psi^{\prime}$ induced up to $H^{0}$ is irreducible.

Now $H^{0} \cap \mathscr{N}_{F}$ is a connected algebraic subgroup of $\mathscr{N}_{F}$. Therefore let us now consider the following situation. Suppose $H_{1}$ and $H_{2}$ are two subgroups of $C_{\infty}$, such that $H^{0} \subseteq H_{1} \subseteq H_{2}$. Suppose also that $H_{i} \cap \mathscr{N}_{F}=G_{i}$ is a connected algebraic subgroup of $\mathscr{N}_{F}$, for $i=1,2$, that $H_{i}=H^{0} \cdot G_{i}$, and that $G_{2}$ normalizes $G_{1}$. Let $\rho_{i}=\operatorname{ind}_{H}^{H_{i}} \psi^{\prime}$ for $i=1,2$. We will show that if $\rho_{1}$ is irreducible, then $\rho_{2}$ is irreducible. In this way, we may conclude after a finite number of steps that

$$
\operatorname{ind}_{H}^{H^{0} \cdot r_{F}} \psi^{\prime}
$$

is irreducible. But since $H^{0} \mathscr{N}_{F}$ is normal in $C_{\infty}$, the same analysis 
as used above to show ind $\mathrm{d}_{H}^{H^{0}} \psi^{\prime}$ irreducible will show ind $\mathrm{d}_{H}^{C_{\infty}} \psi^{\prime}$ irreducible, and we will have shown the sufficiency of the condition of the proposition.

Let $\odot$ be the $\mathrm{Ad}^{*} H_{1}$ orbit in $\hat{J}_{1}$ (where $J_{1}=\log H_{1}$ ) corresponding to $\rho_{1}$. Then, as before, $O$ contains the restriction of $\psi$ to $J_{1}$. Consider the restriction $\sigma_{1}$ of $\rho_{1}$ to $G_{1}$. If $\sigma^{\prime}$ is the set of restrictions of elements of $\bigcirc$ to $\log G_{1}$, then since $\log G_{1}$ is preserved by $\operatorname{Ad} H_{1}$, $\mathcal{O}^{\prime}$ is just the $\mathrm{Ad}^{*} H_{1}$ orbit of the restriction $\psi_{1}$ of $\psi$ to $\log G_{1}$. $\mathcal{O}^{\prime}$ is a union of $\mathrm{Ad}^{*} G_{1}$ orbits, permuted transitively by $\mathrm{Ad}^{*} H_{1} / G_{1}$. We see $\sigma_{1}$ decomposes into a direct integral of representations corresponding to these orbits.

Since $G_{1}$ is normal in $H_{2}, \operatorname{Ad}^{*} H_{2}$ acts on $\log G_{1}$. Suppose for all $h \in H_{2}-H_{1}, \mathrm{Ad}^{*} h\left(\mathscr{O}^{\prime}\right) \cap \mathscr{O}^{\prime}$ is empty. Then $\sigma_{1}$ and $\mathrm{Ad}^{*} h\left(\sigma_{1}\right)$ are disjoint - that is, contain no common irreducible components, since $\mathrm{Ad}^{*} h\left(\sigma_{1}\right)$ will be a direct integral over the representations corresponding to $\mathrm{Ad}^{*} G_{1}$ orbits in $\mathrm{Ad}^{*} h\left(\mathscr{O}^{\prime}\right)$, by the naturality of the correspondence of Proposition 1.1. Then it follows (see [23]) that $\rho_{2}$ is irreducible. Thus we must consider the possibility that $\mathscr{O}^{\prime}$ and $\mathrm{Ad}^{*} h\left(\mathscr{O}^{\prime}\right)$ are not disjoint. Then if $\varphi \in \mathscr{O}^{\prime} \cap \mathrm{Ad}^{*} h\left(\mathcal{O}^{\prime}\right)$ we have $\varphi=\operatorname{Ad}^{*} x\left(\psi_{1}\right)$, with $x \in H_{1}$, and $\varphi=\operatorname{Ad}^{*} h\left(\varphi^{\prime}\right)$ for some $\varphi^{\prime} \in \mathcal{O}^{\prime}$, and $\varphi^{\prime}=\operatorname{Ad}^{*} y\left(\psi_{1}\right)$ for some $y \in H_{1}$. Thus $\mathrm{Ad}^{*} x^{-1} h y\left(\psi_{1}\right)=\psi_{1}$. In particular $\mathrm{Ad}^{*} z^{-1} h y\left(\psi_{1}\right)$ agrees with $\psi$ on $J \cap N_{F}$. Since $\mathrm{Ad}^{*} H(\psi)=\psi+J^{\perp}, \mathrm{Ad}^{*} H\left(\psi_{\left.\right|_{F}}\right)$ consists of all elements in $\hat{N}_{F}$ which agree with $\psi$ on $J \cap N_{F}$. Therefore, we can find $z \in H$ such that $\mathrm{Ad}^{*} z x^{-1} h y\left(\psi_{\mid N_{F}}\right)=\psi_{\left.\right|_{F}}$. Then $z x^{-1} h y \in M^{0} \subseteq H^{0}$. Hence $h \in H_{1}$. Thus always $\operatorname{Ad}^{*} h\left(\mathcal{O}^{\prime}\right) \cap \mathcal{O}^{\prime}$ is empty if $h \in H_{2}-H_{1}$, so the first half of the lemma is complete. Now we prove necessity. We assume $\rho=\operatorname{ind}_{H}^{C_{\infty}} \psi^{\prime}$ is irreducible, and want to show $\operatorname{Ad}^{*} H(\psi)=$ $\psi+J^{\perp}$. As in [17], remark preceding Proposition 12, we must have $\psi^{\prime}+J^{\perp} \subseteq \operatorname{Ad}^{*} C_{\infty}(\psi)=\mathcal{O}$, or else $\rho$ would contain other representations besides the one corresponding to $\mathcal{O}$. So we may assume $\psi+J^{\perp} \subseteq \mathcal{O}$, and then $\rho$ is a factor representation, equivalent to some multiple of the representation corresponding to $\mathcal{O}$.

With this observation, the procedure becomes similar to that followed just above. Suppose $c \in C_{\infty}-H$ is such that $\operatorname{Ad}^{*} c(\psi) \epsilon$ $\psi+J^{\perp}$. Then it is not hard to see that we may find subgroups $G_{1}$ and $G_{2}$ of $C_{\infty}$ such that $H \subseteq G_{1} \subseteq G_{2}, G_{1}$ is e.e. and normal in $G_{2}$ and $G_{2} / G_{1}$ is finite (so $G_{1}$ is also open in $G_{2}$ ), and $c \in G_{2}-G_{1}$. For if $c \in$ $H^{0}$, consider the sequence $\left\{H_{i}\right\}_{i=0}^{\infty}$ of subgroups of $H^{0}$ constructed above, and let $j$ be the smallest integer such that $c \in H_{j}$. Then put $G_{2}=H_{j}, G_{1}=H_{j-1}$. If $c \notin H^{0}$, then let $H^{0} \leqq H_{1}^{0} \subseteq H_{2}^{0} \cdots \subseteq H_{k}^{0}=C_{\infty}$ be a sequence of groups such that $H_{i}^{0} \cap \mathscr{N}_{F}$ is normal in $H_{i+1}^{0}$. Suppose $c \in H_{i+1}^{0}-H_{i}^{0}$. Let $G_{2}$ be generated by $H_{i}^{0}, c$, and a small neighborhood of the origin in $H_{i+1}^{0}$. Then $G_{2}$ is compact modulo $H_{i}^{0}$, and it is clear that one may choose $G_{1} \subseteq G_{2}$ satisfying the stated conditions. 
We will show $\rho_{2}=\operatorname{ind}_{H}^{\sigma_{2}} \psi^{\prime}$ is reducible. We may as well assume $\rho_{1}=\operatorname{ind}_{H}^{G_{1}} \psi^{\prime}$ is irreducible, or we are done. Then, as observed above, if $\psi_{1}$ is the restriction of $\psi$ to $\log G_{1}$, then $\operatorname{Ad}^{*} G_{1}\left(\psi_{1}\right)$ must contain all $\varphi \in \log G_{1}$ which agree with $\psi_{1}($ i.e., $\psi)$ on $J=\log H$. Since $\operatorname{Ad}^{*} c(\psi) \in$ $\psi+J^{\perp}$, we have $\operatorname{Ad}^{*} c\left(\psi_{1}\right) \in \operatorname{Ad}^{*} G_{1}\left(\psi_{1}\right)$, which means $\operatorname{Ad}^{*} c\left(\operatorname{Ad}^{*} G_{1}\left(\psi_{1}\right)\right)=$ $\operatorname{Ad}^{*} G_{1}\left(\psi_{1}\right)$. Since $\operatorname{Ad}^{*} G_{1}\left(\psi_{1}\right)$ corresponds to $\rho_{1}, c$ is in the isotropy group of $\rho_{1}$ under $\operatorname{Ad}^{*} G_{2} / G_{1}$. Hence $\rho_{2}=\operatorname{ind}_{G_{1}}^{G_{2}} \rho_{1}$ cannot be irreducible. So finally $\rho=\operatorname{ind}_{G_{2}}^{\sigma_{\infty}} \rho_{2}$ is reducible and Proposition 1.2 is finished.

An e.e. subgroup $H$ of $C_{\infty}$ subordinate to $\psi \in \hat{V}_{\infty}$ which satisfies the equivalent conditions of Proposition 1.2 will be said to polarize $\psi$.

The next proposition will allow us not only to find groups from which to induce representations of $C_{\infty}$, but of $\mathscr{S}_{F}$ as well. It will have several important consequences for the representation theory of $\mathscr{S}_{F}$. Before proving it, we record an elementary result.

Let $\mathscr{\mathscr { L }}_{n}$ denote the $n$th order Heisenberg group over $F$. That is $\mathscr{\mathscr { L }}_{n}$ is a two-step nilpotent connected unipotent group, of dimension $2 n+1$, and with one-dimensional center $\underline{\underline{\underline{z}}}$. The Heisenberg groups are basic to Kirillov theory for nilpotent and solvable groups, and are important in other connections too. Thus for future reference, we spell out explicitly some details of its representation theory. These are consequences of Propositions 1.1 and 1.2, but have essentially been known at least since [24]. We leave the proof as an exercise.

LEMMA 1.2. Let $\rho$ be an irreducible representation of $\mathscr{H}_{n F}$. Then if $\rho$ is trivial on $\mathcal{Z}_{F}$, $\rho$ is one-dimensional. If $\rho$ is nontrivial on $\mathscr{Z}_{F}$, then $\rho$ restricted to $\mathscr{Z}_{F}$ is a multiple of some linear character $\varphi_{\rho}$. In turn, $\varphi_{\rho}$ determines $\rho$. If $P$ is any subgroup of $\mathscr{H}_{n F}$, such that $\mathcal{Y}_{F} \subseteq P$ and if $\sigma$ is a representation of $P$ and restriction of $\sigma$ to $\mathscr{Z}_{F}$ is a multiple of $\varphi_{\rho}$, then inducing $\sigma$ up to $\mathscr{H}_{n F}$ gives $a$ multiple of $\rho$. It is possible to induce $\rho$ from a one-dimensional character of $P$ if and only if $P / \operatorname{ker} \varphi_{\rho}$ is a maximal abelian subgroup of $\mathscr{H}_{n F} / \operatorname{ker} \varphi_{\rho}$. If $P$ is an algebraic subgroup of $\mathscr{H}_{n F}$, then this is equivalent to saying $P$ is maximal abelian in $\mathscr{H}_{n F}$. In this case, $\operatorname{dim} P=n+1$. Any $P$ such that $P / \operatorname{ker} \varphi$ is abelian is contained in a maximal group of this type.

Now consider the action $\mathrm{Ad}^{*} \mathscr{S}_{F}$ of $\mathscr{S}_{F}$ on $\hat{N}_{F}$. As is well-known, this action is algebraic. This may be seen as follows. Take any character $\chi$ of $F$. Then using $\chi$, one may define a homomorphism $\alpha: N_{F}^{*} \rightarrow \hat{N}_{F}$, where $\underline{\underline{N}}^{*}$ is the vector space dual of $\underline{\underline{N}}$. The formula defining $\alpha$ is $\alpha\left(n^{*}\right)(n)=\chi\left(n^{*}(n)\right)$, where $n \in N_{F}$ and $n^{*} \in N_{F}^{*}$. It may be verified (see [37]) that $\alpha$ is in fact an isomorphism of topological groups. Moreover, if $\mathrm{Ad}^{*} \mathscr{\mathscr { S }}$ denotes the action of $\mathscr{S}$ on $\underline{N}^{*}$ dual to $\mathrm{Ad} \mathscr{\mathscr { S }}$ on $\underline{\underline{N}}$ (the co-adjoint action) then the diagram 


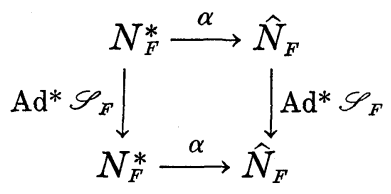

commutes. This shows the action $\mathrm{Ad}^{*} \mathscr{S}_{F}$ on $\hat{N}_{F}$ is indeed algebraic and further justifies the continuing ubiquitous use of $\mathrm{Ad}^{*}$ to denote these actions.

Now consider a point $\psi \in \hat{N}_{F}$. Let $\mathscr{S}_{\psi}$ be the isotropy group of $\psi$ under $\mathrm{Ad}^{*} \mathscr{S}_{F}$. From the above, we see $\mathscr{S}_{\psi}$ is in fact the group of $F$-rational points of an algebraic group $\mathscr{S}_{\psi}$ defined over $F$. Of course, $\mathscr{L}_{\psi}$ is not necessarily connected.

Proposition 1.3. Take $\psi \in \hat{N}_{F}$. Then there are algebraic subgroups $\mathscr{L}^{1} \subseteq \mathscr{L}^{2} \subseteq \mathscr{L}^{3}$, all normalized by $\mathscr{L}_{\psi}$, and having the following properties. $\mathscr{S}_{\psi} \cap \mathscr{N} \subseteq \mathscr{L}^{2}$. Both $\mathscr{L}^{1}$ and $\underline{\mathscr{L}}^{2}$ are normal in $\mathscr{L}^{3}$, and $\mathscr{L}^{3} / \mathscr{L}^{1}$ is a Heisenberg group with $\mathscr{L}^{2} / \mathscr{L}^{1}$ as its one-dimensional center. The restriction of $\psi \circ \log$ to $\mathscr{L}_{F}^{2}$ is a nontrivial linear character $\varphi$, trivial on $\mathscr{L}_{F}^{1}$. Thus, if $\mathscr{\mathscr { P }} \subseteq \mathscr{L}^{3}$ is an algebraic subgroup, with $\mathscr{\mathscr { P }} \supseteqq \mathscr{L}^{2}$, then if $\underline{\underline{P}} / \mathscr{L}^{2}$ is abelian, then $\mathscr{P}_{F}$ is subordinate to $\psi$. If $\underline{\underline{\mathscr{P}}} / \mathscr{L}^{1}$ is maximal abelian in $\underline{\mathscr{L}}^{3} / \mathscr{L}^{1}$, then $\mathscr{P}_{F}$ polarizes $\psi$. Thus, any subgroup $G$ of $\mathscr{L}_{F}^{3}$, containing $\mathscr{L}_{F}^{2}$, and such that $G / \operatorname{ker} \varphi$ is maximal abelian in $\mathscr{L}_{F}^{3} / \operatorname{ker} \varphi$, polarizes $\psi$ and induces the irreducible representation corresponding to $\operatorname{Ad}^{*} \mathscr{N}_{F}(\psi)$.

Furthermore, one may choose groups $G^{1}, i=1,2,3$, as follows. First, $\mathscr{L}_{F}^{2} \subseteq G^{1} \subseteq G^{2} \cong G^{3} \subseteq \mathscr{L}_{i F}^{3}$. Second, $G^{2}$ polarizes $\psi$, and $G^{1}$ and $G^{3}$ are normalized by $\mathscr{S}_{\psi}$. Third, $G^{1}$ has finite index in $G^{3}$. Finally, $G^{2}$ is normalized by a subgroup of $\mathscr{S}_{\psi}$, of finite index prime to $p$. If $\mathscr{S}$ is split (or simply if the action $\mathrm{Ad}^{*} \mathscr{S}_{\psi}$ splits over $F$ ), then one may take $\mathscr{L}^{2}=\mathscr{L}^{3}$, in which case $\mathscr{L}_{F}^{3}$ itself is subordinate to and polarizes $\psi$.

REMARK. Thus in the $p$-adic case too the relative complexity of the nonsplit case is evident. The comparison to the real case is quite direct and is the more interesting in the light of known ways to realize discrete series representations of semisimple groups over real and $p$-adic fields. There is also a parallel result for algebraic solvable groups over finite fields (see, for example, [19]) which may be compared with the classically known facts ([12]) that given a finite group $G$, for every representation of $G$ to be monomial, it is necessary that $G$ be solvable and sufficient that $G$ be supersolvable. 
less immediately from Proposition 1A of [19] and the facts about the Heisenberg group recorded in Lemma 1.2. We will not go into details. We should perhaps mention that the definition of polarizing, given here on the basis of Proposition 1.2, is the same as that given in [19] for the case of an algebraic subgroup of a unipotent group. For if $\mathscr{\mathscr { P }} \subseteq \mathscr{N}$ is an algebraic subgroup, with $\operatorname{dim} \mathscr{\mathscr { P }}=1 / 2(\operatorname{dim} \mathscr{\mathscr { N }}=$ $\left.\operatorname{dim}\left(\mathscr{S}_{\psi \cap \mathscr{N}}\right)\right)$, and $\mathscr{P}_{F}$ is subordinate to $\psi$, then $\operatorname{Ad}^{*} \mathscr{P}_{F}(\psi)$ is open and (Zariski!) closed in $\psi+\left(\log \mathscr{P}_{F}\right)^{\perp}$. (One uses on $\hat{N}$ the structure of variety over $F$ defined via the map $\alpha$ given above.)

The third paragraph of the proposition follows easily by inspection of the proof of Proposition 1A of [19].

We will prove the second paragraph of the proposition. Since $\mathscr{S}_{\psi} \cap \mathscr{N}_{F} \subseteq \mathscr{L}_{F}^{2}, \mathscr{S}_{\psi}$ acts (by conjugation) completely reducibly on $\mathscr{L}_{F}^{3} / \mathscr{L}_{F}^{2}$ (which we may regard as a vector space over $F$, since it is abelian). Write $\mathscr{L}_{F}^{3} / \mathscr{L}_{F}^{2}=M=\bigoplus_{i=1}^{k} M_{i}$, where the $M_{i}$ are irreducible subspaces under the action of $\mathscr{S}_{\psi}$. Let us once again denote this action by Ad $\mathscr{S}_{\psi}$.

As is well-known (see [19]), if in the Heisenberg group $\mathscr{H}_{n F}$ if one identifies $\mathscr{Z}_{F}$ with the additive group of $F$, then taking commutators induces a nondegenerate, antisymmetric (i.e., symplectic) form on $\mathscr{\mathscr { H }}_{n} / \mathscr{2}$. Hence in our situation we have a symplectic form $\langle$,$\rangle on M$, and since Ad $\mathscr{S}_{\psi}$ is an action derived from automorphisms of $\mathscr{L}_{F}^{3}$ or, more to the point, of $\mathscr{L}_{F}^{3} / \mathscr{L}_{F}^{1}$, this symplectic form is preserved by Ad $\mathscr{S}_{\psi}$. Thus in each $M_{i}$, the radical of the restriction of $\langle$,$\rangle is invariant by Ad \mathscr{S}_{\psi}$, and consequently either $\langle$,$\rangle remains$ nondegenerate when restricted to a given $M_{i}$, or $M_{i}$ is completely isotropic with respect to $\langle$,$\rangle . Suppose some M_{i}$ is isotropic. Then for some $j,\left\langle M_{i}, M_{j}\right\rangle \neq\{0\}$. Since the annihilator with respect to $\langle$, of $M_{i}$ in $M_{j}$ is invariant, it is zero, since $M_{j}$ is irreducible. Reversing $i$ and $j$ and repeating the argument, we see $\operatorname{dim} M_{i}=\operatorname{dim} M_{j}$, and that $\langle$,$\rangle puts M_{i}$ and $M_{j}$ in duality. Now

$$
M^{\prime}=\left\{m \in M,\left\langle m, M_{i}+M_{j}\right\rangle=0\right\}
$$

is invariant by Ad $\mathscr{S}_{\psi}$, so we may as well assume that $M^{\prime}=\bigoplus_{l \neq i, j}^{k} M_{l}$. Now let $\tilde{\mathscr{L}}_{F}^{3}$ be the inverse image in $\mathscr{L}_{F}^{3}$ of $M^{\prime} \oplus M_{i}$ and let $\tilde{\mathscr{L}}_{F}^{2}$ be the inverse image of $M_{i}$. Then it is clear that $\tilde{\mathscr{L}}_{F}^{2}$ is still subordinate to $\psi$. Let $\tilde{\mathscr{L}}_{F}^{1}$ be the largest algebraic subgroup of $\tilde{\mathscr{L}}_{F}^{2}$ on which $\psi \circ \log$ is trivial. Then we see that $\tilde{\mathscr{L}}_{F}^{1}, \tilde{\mathscr{L}}_{F}^{2}$, and $\tilde{\mathscr{L}}_{F}^{3}$ are the $F$ rational points of algebraic subgroups which also satisfy the conditions of the first paragraph of the proposition. Hence we may restrict our attention to the case when $M=\bigoplus_{i=1}^{k} M_{i}$, and $\langle$,$\rangle is nondegenerate$ on each $M_{i}$.

Now $\mathscr{S}_{\psi} /\left(\mathscr{S}_{\psi} \cap \mathscr{N}_{F}\right)$ is abelian, so $\mathrm{Ad}^{*} \mathscr{S}_{\psi}$ acting on $M_{i}$ is an 
action of an abelian group. Thus we may apply the lemma of [19] to conclude there is a field extension $F_{i}$ of $F$, and a quadratic extension $F_{i}^{\prime}$ of $F_{i}$, and a homomorphism $h_{i}: \mathscr{S}_{\psi} \rightarrow F_{i}^{\prime x}$, with image in the kernel of the norm map from $F_{i}^{\prime}$ to $F_{i}$, and an isomorphism $\mu_{i}: M_{i} \rightarrow F_{i}^{\prime}$, such that

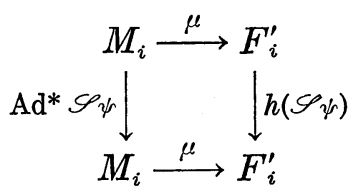

commutes. (The action on the right is by multiplication in $F_{i}^{\prime}$.) Moreover, there is an element $c \in \operatorname{ker} \operatorname{tr}\left(F_{i}^{\prime} / F_{i}\right)$ such that

$$
\left\langle\mu^{-1}(x), \mu^{-1}(y)\right\rangle=\operatorname{tr}\left(F_{i} / F\right)(c(\tau(x) y-\tau(y) x)),
$$

where $\operatorname{tr}$ denotes trace and $\tau$ is the Galois automorphism of $F_{i}^{\prime}$ over $F_{i}$.

Now to obtain $\langle$,$\rangle , we have identified \mathscr{L}_{F}^{2} / \mathscr{L}_{F}^{1}$, the center of $\mathscr{L}_{F}^{3} / \mathscr{L}_{F}^{1}$, with $F$. In this identification, we may suppose that $R$ is identified to the largest $R$-module in the kernel of $\psi \circ \log$ factored to $\mathscr{L}_{F}^{2} / \mathscr{L}_{F}^{1}$. For any $R$-module $Z \subseteq M$, put $Z^{*}=\{m \in M,\langle m, Z\rangle \subseteq R\}$. Then since $\langle$,$\rangle is derived from taking commutators, we see that$ the inverse image of $Z$ in $\mathscr{L}_{F}^{3}$ is abelian modulo ker $\psi \circ \log$ if and only if $Z \subseteq Z^{*}$, and maximal abelian if and only if $Z=Z^{*}$. Suppose $Z=\bigoplus_{i=1}^{k} Z_{i}$, where $Z_{i}=Z \cap M_{i}$. Then also $Z^{*}=\bigoplus_{i=1}^{k} Z_{i}^{*}$, with $Z_{i}^{*}=$ $Z^{*} \cap M_{i}$.

Now suppose $\mu_{i}\left(Z_{i}\right)=\pi_{i}^{\prime a} R_{i}^{\prime}$, where $\pi_{i}^{\prime}$ is a prime of $F_{i}^{\prime}$, and $R_{i}^{\prime}$ is the ring of integers of $F_{i}^{\prime}$. Then a calculation using the explicit form of $\langle,\rangle \circ \mu_{i}^{-1}$ shows that $\mu_{i}\left(Z_{i}^{*}\right)$ is also an $R_{i}^{\prime}$-module, and hence $\mu_{i}\left(Z_{i}^{*}\right)=\pi_{i}^{\prime b} R_{i}^{\prime}$. Moreover, it is easy to see that as $a$ varies, the sum $a+b=d$ remains constant. If $d$ is even, we may then choose $Z_{i}$ so that $a=b$, and then $Z_{i}=Z_{i}^{*}$. If $d$ is odd, then we can arrange $a=b+1$, so that $\mu_{i}\left(Z_{i}\right)=\pi_{i}^{\prime} \mu_{i}\left(Z_{i}^{*}\right)$. In either case, if $s \in \mathscr{S}_{\psi}$ is such that $h_{i}(s) \in 1+\pi_{i}^{\prime} R_{i}^{\prime}$, then Ad $s$ leaves invariant any $Z_{i}^{\prime}$ such that $Z_{i} \subseteq Z_{i}^{\prime} \leqq Z_{i}^{*}$. But the kernel of the norm map from $F_{i}^{\prime}$ to $F_{i}$ is in particular a subgroup of $R_{i}^{\prime x}$, the units of $R_{i}^{\prime}$, and $1+\pi_{i}^{\prime} R_{i}^{\prime}$ is a subgroup of $R_{i}^{\prime x}$ of finite index prime to $p$.

Now we see that if we choose $Z^{1}=\bigoplus_{i=1}^{k} Z_{i}$, where the $Z_{i}$ are chosen as just above, and then put $Z^{3}=Z^{1^{*}}$, and let $Z^{2}$ satisfy $Z^{1} \subseteq$ $Z^{2} \subseteq Z^{3}$ and $Z^{2}=Z^{2^{*}}$, then if $G^{i}, i=1,2,3$, is the inverse image of $Z^{i}$ in $\mathscr{L}_{F}^{3}$, the $G^{i}$ satisfy the requirements of the proposition, and we are done.

Now we spell out some consequences of the above proposition. Again choose $\psi \in \hat{N}_{F}$. Since $\mathscr{S}_{\psi}$ is algebraic, we may by [8], write 
$\mathscr{S}_{\psi}=\mathscr{T}_{\psi} \cdot \mathscr{N}_{\psi}$ where $\mathscr{N}_{\psi}=\mathscr{S}_{\psi} \cap \mathscr{N}_{F}$, and $\mathscr{T}_{\psi}$ is the group of $F$ rational points of a reductive group defined over $F$. $\mathscr{T}_{\psi}$ is abelian, since $\mathscr{T}_{\psi} \cong \mathscr{S}_{\psi} / \mathscr{N}_{\psi} \subseteq \mathscr{S}_{F} / \mathscr{N}_{F}$. If $\mathscr{S}_{\psi}$ were connected, then $\mathscr{T}_{\psi}$ would be a maximal torus of $\mathscr{S}_{\psi}$.

Since $\mathscr{S}_{\psi}$ is a semidirect product we may apply to $\mathscr{S}_{F}$ the Mackey theory for representations of a semidirect product. References for the general theorems, whose specializations to the present case we will now relate, are [5] and [23]. We note here at the beginning that the preconditions for application of the theory, e.g., that $\mathscr{N}_{F}$ be type $\mathrm{I}$, and that the action $\mathrm{Ad}^{*} \mathscr{S}_{F}$ of $\hat{\mathscr{N}}_{F}$ be smooth, in the sense of Mackey, (which is much less smooth than most people demand) are easily checked to be true. Also, we remark that we could, by special arguments, avoid the use of Mackey's theory, since we already have complete control over the open normal subgroup $C_{\infty}$ of $\mathscr{S}_{F}$. However, since the general theory is available, it would seem egregious to ignore it.

If $\rho$ is an irreducible representation of $\mathscr{S}_{F}$, then according to Mackey's theory, the restriction of $\rho$ to $\mathscr{N}_{F}$ breaks up into a direct integral over representations all belonging to a given $\mathrm{Ad}^{*} \mathscr{S}_{F}$ orbit $\mathcal{O}$ in $\hat{\hat{N}_{F}}$. We say that $\rho$ lies above $\mathcal{O}$. This terminology gains context from the fact that by associating $\odot$ to $\rho$ we get a continuous $\operatorname{map} r: \hat{\mathscr{S}}_{F} \rightarrow \hat{\mathscr{N}}_{F} / \mathrm{Ad}^{*} \mathscr{S}_{F}$. The set $r^{-1}(\mathscr{O})$, consisting of (primitive ideals attached to) representations lying above $\mathcal{O}$, is called the fiber over $\mathcal{O}$.

Mackey's theory permits us to describe $r^{-1}(\mathcal{O})$. First, let us identify $\hat{\mathscr{N}}_{F} / \operatorname{Ad}^{*} \mathscr{S}_{F}$. By Proposition 1.1, we have the homeomorphism $\beta: \hat{N}_{F} / \mathrm{Ad}^{*} \hat{N}_{F} \rightarrow \hat{\mathscr{N}}_{F}$. Since $\beta$ is natural, it follows that we also have a homeomorphism $\bar{\beta}: \hat{N}_{F} / \operatorname{Ad}^{*} \mathscr{S}_{F} \rightarrow \hat{\mathscr{N}}_{F} / \operatorname{Ad}^{*} \mathscr{S}_{F}$. Thus $\bar{\beta}^{-1}(\mathcal{O})$ is an $\mathrm{Ad}^{*} \mathscr{S}_{F}$ orbit in $\hat{N}_{F}$. Call it $\widetilde{\mathcal{O}}$. Pick $\psi \in \tilde{\mathscr{O}}$, and let $\tilde{\mathscr{O}}_{1}=$ $\operatorname{Ad}^{*} \mathscr{N}_{F}(\psi)$. Then $\beta\left(\widetilde{\mathscr{O}_{1}}\right)=\sigma$, is an irreducible representation of $\mathscr{N}_{F}$, and is a point in $\mathscr{O}$. For Mackey's theory, we must identify the isotropy group $\mathscr{S}_{\sigma}$ of $\sigma$ under $\operatorname{Ad}^{*} \mathscr{S}_{F}$. By the naturality of $\beta$, this is the subgroup of $\mathscr{S}_{F}$ of elements $s$ such that $\operatorname{Ad}^{*} s\left({\widetilde{O_{1}}}_{1}\right)=\tilde{\mathscr{O}}_{1}$. This in turn is the group of $s$ such that $\operatorname{Ad}^{*} s(\psi) \in \widetilde{\mathscr{O}}_{1}$. Thus if $s \in \mathscr{S}_{\sigma}$, there is $n \in \mathscr{N}_{F}$ such that $\operatorname{Ad}^{*} n s(\psi)=\psi$. Therefore $\mathscr{S}_{o}=\mathscr{S}_{\psi} \cdot \mathscr{N}_{F}=$ $\mathscr{T}_{\psi} \times_{8} \mathscr{N}_{F}$.

We will show shortly on the basis of Proposition 1.3 that $\sigma$ can be extended to a representation $\sigma^{\prime}$ of $\mathscr{S}_{\sigma}$. That is, there is an irreducible representation $\sigma^{\prime}$ of $\mathscr{S}_{\sigma}$ such that the restriction of $\sigma^{\prime}$ to $\mathscr{N}_{F}$ is exactly $\sigma$ (and not a multiple of $\sigma$ ). This is the essential point. Then Mackey's theory shows that every representation of $\mathscr{S}_{\psi}$ lying above $\mathcal{O}$ is uniquely of the form $\operatorname{ind}_{S_{\sigma}^{F}}^{S_{F}} \mu \otimes \sigma^{\prime}$, where $\mu$ 
is an irreducible representation of $\mathscr{S}_{\sigma}$, trivial on $\mathscr{N}_{F}$. Thus $\mu$ is essentially a representation of $\mathscr{T}_{\psi}$, and since $\mathscr{T}_{\psi}$ is abelian, $\mu$ is one dimensional. Moreover, we note that $\mu$ is the restriction to $\mathscr{S}_{\sigma}$ of a linear character $\nu$ of $\mathscr{S}_{F} / \mathscr{N}_{F}$, and a simple check shows $\operatorname{ind}_{\mathscr{S}_{\sigma}^{F}}^{S_{F}} \mu \otimes \sigma^{\prime}=\nu \otimes \sigma^{\prime}=\nu \otimes$ ind $_{\mathscr{S}_{\sigma}^{F}}^{\mathscr{S}_{F}} \sigma^{\prime}$. Thus, we see that we may parametrize $r^{-1}(\mathcal{O})$ by elements from $\hat{\mathscr{T}} \psi$, or more canonically, by elements of $\hat{\mathscr{T}}_{F} / \mathscr{T}_{\psi}^{\perp}$, where $\mathscr{T}_{\psi}^{\perp} \subseteq \hat{\mathscr{T}}_{F}$ is the subgroup of characters of $\mathscr{T}_{F}$ annihilating $\mathscr{T}_{\psi}$. Of course, the parametrization depends on the choice of base-point, that is, on the choice of $\sigma^{\prime}$. For if $\sigma^{\prime}$ extends $\sigma$, then so does $\mu \otimes \sigma^{\prime}$ for any $\mu \in \hat{\mathscr{T}} \psi$. We will consider later on the problem of choosing the "correct" extension of $\sigma$.

For any locally compact group $G$, let $\hat{G}_{1}$ be the group of linear characters of $G$. Then $\widehat{G}_{1}$ acts as a transformation group on $\hat{G}$ via tensor product. Thus if $\sigma$ is an irreducible representation of $G$, and $\phi$ is a linear character, we define " $\rho$ translated by $\phi$ " to be $\phi \otimes \rho$. In particular, for our $\mathscr{S}_{F}$, we have $\hat{\mathscr{T}}_{F} \subseteq\left(\hat{\mathscr{S}}_{F}\right)_{1}$, so $\hat{\mathscr{T}}_{F}$ acts on $\hat{\mathscr{S}}_{F}$. By what we have seen just above, we may describe the sets $r^{-1}(\mathscr{O})$ for $\mathcal{O}$ an $\mathrm{Ad}^{*} \mathscr{S}_{F}$ orbit in $\hat{\mathscr{N}_{F}}$, as being precisely the orbits for this action of $\mathscr{T}_{F}$. Also, the isotropy group of any point in $r^{-1}(\mathcal{O})$ under this action is, as we have noted, $\mathscr{T}_{\psi}{ }^{\perp}$. This completes our description of $r^{-1}(\mathbb{O})$.

Now let us show that $\sigma$ may be extended to a representation of $\mathscr{S}_{\sigma}$. Recall that $\psi$ is a point in $\hat{\mathscr{O}}_{1}=\beta^{-1}(\sigma)$, and $\mathscr{S}_{\sigma}=\mathscr{T}_{\psi} \times \mathscr{N}_{s}$. Let $G^{i}, i=1,2,3$, be the groups described in the second paragraph of Proposition 1.3. We then see from Proposition 1.2 that $\sigma$ is induced from the restriction of $\psi \circ \log$ to $G^{2}$. Let $\sigma_{1}=\operatorname{ind}_{G^{2}}^{G^{3}}(\psi \circ \log )$. Then clearly $\sigma$ is induced from $\sigma_{1}$. But $G^{3}$ is normalized by $\mathscr{S}_{\psi}$ and $\mathscr{S}_{\psi} \cdot G_{3}=$ $\mathscr{T}_{\psi} \mathrm{X}_{s} G^{3}$. Moreover, $\sigma_{1}$ is obviously invariant under $\mathrm{Ad}^{*} \mathscr{S}_{\psi} \cdot G_{3}$. Suppose we can extend $\sigma_{1}$ to a representation $\sigma_{1}^{\prime}$ of $\mathscr{S}_{\psi} \cdot G_{3}$. Then clearly the representation of $\mathscr{S}_{\sigma}$ induced from $\sigma_{1}^{\prime}$ extends $\sigma$. Therefore we are reduced to extending $\sigma_{1}$ from $G_{3}$ to $\mathscr{T} \psi X_{s} G_{3}$. This is a classical problem, which in the context of locally compact groups has been considered by Mackey. Again we refer to [5] and [23] for details. The obstruction to extending $\sigma_{1}$ (the "Mackey obstruction") is a certain element of $H^{2}\left(\mathscr{T}_{\psi}, \boldsymbol{T}\right)$, the second cohomology of $\mathscr{T}_{\psi}$ with coefficients in the circle group. This element has finite order dividing the degree of $\sigma_{1}$, which in this case is a power of $p$ ( $p$ being the residual characteristic of $F$ ). We will show that it also has order prime to $p$, and hence is trivial.

Let $\mathscr{R} \subseteq \mathscr{T}_{\psi}$ be the normalizer of $G^{2}$ in $\mathscr{T}_{\psi}$. By Proposition 1.4, $\mathscr{T}_{\psi} / \mathscr{R}$ is finite of order prime to $p$. Now since $\mathrm{Ad}^{*} \mathscr{T}_{\psi}$ leaves $\psi \in \hat{N}_{F}$ fixed, Ad* $\mathscr{R}$ leaves $\psi \circ \log \in \hat{G}^{2}$ fixed.

Let us briefly recall how the Mackey obstruction arises. Since 
$\mathrm{Ad}^{*} \mathscr{T}_{\psi}$ leaves $\sigma_{1}$ fixed, there is for each $t \in \mathscr{T}_{\psi}$ a unitary operator $U(t)$ on the space of $\sigma_{1}$ which satisfies $\sigma_{1}\left(t^{-1} g t\right)=U(t)^{-1} \sigma_{1}(g) U(t)$ for $g \in G^{3}$. The operator $U(t)$ is determined up to scalar multiples. Thus if we select for each $t \in \mathscr{T}_{\psi}$ some $U(t)$ having the above property, then for each pair $t_{1}, t_{2} \in \mathscr{T}_{\psi}$ there is a constant $c\left(t_{1}, t_{2}\right)$ such that $U\left(t_{1}\right) U\left(t_{2}\right)=c\left(t_{1}, t_{2}\right) U\left(t_{1} t_{2}\right)$. The function $c\left(t_{1}, t_{2}\right)$ is a cocycle defining the Mackey obstruction. (In the present context, where $\sigma_{1}$ is finite dimensional, $c\left(t_{1}, t_{2}\right)$ can be chosen to be locally constant.)

Now since $\mathrm{Ad}^{*} \mathscr{R}$ leaves $\psi \circ \log$ on $G^{2}$ invariant, it follows from the definition of $\sigma_{1}$ that for $t \in \mathscr{R}$ and $g \in G^{3}, \sigma_{1}\left(t^{-1} g t\right)=\sigma_{1}(g)$. Thus we may choose $U(t)$, as defined above, to be identically one on $\mathscr{R}$. This extends $\sigma_{1}$ to $\mathscr{R} \times_{s} G^{3}$. Since the extended representation is trivial on $\mathscr{R}$, we see we are actually reduced to the problem of extending $\sigma_{1}$ factored to $G^{3} / \operatorname{ker} \sigma_{1}$ to $\left(\mathscr{T}_{\psi} / \mathscr{R}\right) \times_{s}\left(G^{3} / \operatorname{ker} \sigma_{1}\right)$. The obstruction to doing this is in $H^{2}\left(\mathscr{T}_{\psi} / \mathscr{R}, T\right)$. As is well-known, the cohomology groups of a finite group have exponent dividing the order of the group. Thus our Mackey obstruction has order dividing the order $\mathscr{T} \psi / \mathscr{R}$, which is prime to $p$, as we claimed. This completes the demonstration that the representation $\sigma$ of $\mathscr{N}_{F}$ can be extended to $\mathscr{S}_{0}$.

Having described the irreducible representations of $\mathscr{S}_{F}$, we draw some conclusions about harmonic analysis on $\mathscr{S}_{F}$. Again let $\mathcal{O} \subseteq$ $\hat{\mathscr{N}}_{F}$ be an $\mathrm{Ad}^{*} \mathscr{S}_{F}$ orbit, and choose $\sigma \in \mathcal{O}$, and let $\mathscr{S}_{\sigma}$ be the isotropy group of $\sigma$ under $\mathrm{Ad}^{*} \mathscr{S}_{F}$. Then we have shown any representation $\rho \in r^{-1}(\mathcal{O})$, where $r: \hat{\mathscr{S}}_{F} \rightarrow \hat{\mathscr{N}}_{F} / \mathrm{Ad}^{*} \mathscr{S}_{F}$ is the map described above, is induced from an extension of $\sigma$ to $\mathscr{S}_{\sigma}$. It follows that the restriction of $\rho$ to $\mathscr{N}_{F}$ is the direct integral over $\mathscr{S}_{F} / \mathscr{S}_{\sigma}$ of the representations $\mathrm{Ad}^{*} s(\sigma)$, for $s \in \mathscr{S}_{F} / \mathscr{S}_{\sigma}$. By definition of $\mathscr{S}_{\sigma}$, all these representations are distinct, so each occurs only once in the integration. This means the restriction of $\rho$ to $\mathscr{N}_{F}$ is multiplicity free. (In this situation, this means that the algebra of operators on the space of $\rho$ which commute with $\rho(n)$ for all $n \in \mathscr{N}_{F}$ is abelian.)

We can make this more precise. We may take $\sigma^{\prime}$, and induce it first from $\mathscr{S}_{\sigma}$ to $\mathscr{S}_{\sigma} \cdot C_{\infty}$, obtaining a representation $\rho_{1}^{\prime}$, which will be an extension to $\mathscr{S}_{\sigma} \cdot C_{\infty}$ of an irreducible representation $\rho_{1}$ of $C_{\infty}$. We may then take $\rho_{1}^{\prime}$ and induce it all the way up to $\mathscr{S}_{\sigma}$ to get $\rho$. Since $C_{\infty}$ is open in $\mathscr{S}_{F}$, the restriction to $C_{\infty}$ of $\rho$ will contain $\rho_{1}$ discretely, and since $\rho$ is already multiplicity-free on $\mathscr{N}_{F}$, it follows that the restriction to $C_{\infty}$ of $\rho$ is a discrete direct sum of representations $\mathrm{Ad}^{*} s\left(\rho_{1}\right)$, each occurring once.

Since $C_{\infty}$ is C.C.R., it follows immediately that there are compact operators in the range of $\rho\left(C_{c}^{\infty}\left(\mathscr{S}_{F}\right)\right)$. From [5] it follows that $\mathscr{S}_{F}$ must be type I, as was only to be expected. 
We may also extend this analysis to derive criteria for $\rho$ to be C.C.R., and for $\mathscr{S}_{F}$ to be C.C.R. See [24]. As noted there $\rho$ is C.C. $R$ if and only if $\rho\left(C_{c}^{\infty}\left(\mathscr{S}_{F}\right)\right)$ consists of finite rank operators, and this is so if and only if $\rho\left(C_{c}^{\infty}\left(C_{i}\right)\right)$ consists of finite rank operators, for any fixed $C_{i}$ as in Lemma 1.1, since $C_{c}^{\infty}\left(\mathscr{S}_{F}\right)$ is spanned by translates in $\mathscr{S}_{F}$ of functions supported in $C_{i}$. This just means $\rho$ restricted to $C_{\infty}$ is C.C.R. But now, applying Fell's criterion [13], this is so if and only if $\operatorname{Ad}^{*} \mathscr{S}_{F}\left(\rho_{1}\right)$ is closed in $\hat{C}_{\infty}$. From the fact that $\beta: \hat{V}_{\infty} / \mathrm{Ad}^{*} C_{\infty} \rightarrow \hat{C}_{\infty}$ of Proposition 1.1 is a homeomorphism and natural with respect to $\mathrm{Ad}^{*} \mathscr{S}_{F}$, we see that if $\rho_{1}=\beta\left(\hat{\mathcal{O}_{1}}\right)$, and $\varphi_{1} \in \overline{\mathscr{O}}_{1}$, then $\rho$ is C.C.R. if and only if $\operatorname{Ad}^{*} \mathscr{S}_{F}(\varphi) \subseteq \hat{V}_{\infty}$ is closed.

Now $\mathscr{S}_{F}$ is by definition C.C.R. if and only if all $\rho \in \hat{\mathscr{S}}_{F}$ are C.C.R. This by the above means $\mathrm{Ad}^{*} \mathscr{S}_{F}\left(\varphi_{1}\right)$ is closed for any $\varphi_{1} \in$ $\hat{V}_{\infty}$. Since the kernel of the restriction map from $\hat{S}_{F}$ to $\hat{V}_{\infty}$ is compact (recall $S_{F}$ is the Lie algebra of $\mathscr{S}_{F}$ ), this is the same as to say $\mathrm{Ad}^{*} \mathscr{S}_{F}(\varphi)$ is closed for any $\varphi \in \widehat{S}_{F}$.

Now by [9], if $\mathscr{T}$ is a maximal torus of $\mathscr{L}$ defined over $F$, then we may write $\underline{\underline{\mathscr{T}}}=\underline{\mathscr{I}}_{a} \cdot \mathscr{\mathscr { T }}_{s}$ where $\mathscr{I}_{a}$ is the "anisotropic part" of $\mathscr{T}$, and $\mathscr{T}_{s}$ is the "split part" of $\mathscr{\mathscr { T }}_{.} \mathscr{\mathscr { T }}_{a}$ is characterized as having no characters (in the sense of algebraic groups) defined over $F$, while the characters of $\mathscr{T}$ defined over $F$ restrict to a subgroup of finite index the characters of $\mathscr{\mathscr { T }}_{s}$. Both $\underline{\mathscr{T}}_{a}$ and $\underline{\mathscr{T}}_{s}$ are defined over $F$, and $\mathscr{\mathscr { T }}_{a} \cap \underline{\underline{\mathscr{T}}}_{s}$ is finite.

I claim that $\mathscr{S}_{F}$ is C.C.R. if and only if $\mathscr{T}_{s}$ is central in $\mathscr{S}$. For suppose $\underline{\mathscr{T}}_{s}$ is not central. Then the action $\mathrm{Ad}^{*} \mathscr{T}_{s F}$ on $\hat{S}_{F}$ is nontrivial, and it is diagonalizable over $F$. Thus the eigenvectors for $\mathrm{Ad}^{*} \mathscr{T}_{s F} \operatorname{span} \hat{S}_{F}$. Let $\varphi$ be one such eigenvector. Then $\operatorname{Ad}^{*} \mathscr{S}_{F}(\varphi)$ contains the origin in its closure, and so is not closed. On the other hand, suppose $\mathscr{\mathscr { T }}_{8}$ is central. Then the maximal tori of the adjoint group of $\mathscr{S}$ are anisotropic over $F$. This implies Ad $\mathscr{S}_{F}$ is a compact extension of $\mathrm{Ad} \mathscr{N}_{F}$ (see [9]). Since all $\mathrm{Ad}^{*} \mathscr{N}_{F}$ orbits in $\widehat{S}_{F}$ are closed, so will all $\mathrm{Ad}^{*} \mathscr{S}_{F}$ orbits be closed. This establishes the stated criterion that $\mathscr{S}_{F}$ be C.C.R. Again, one has the complete analogue of the real situation. See [5].

We now summarize the the results established in the discussion since Proposition 1.3 in the following portmanteau theorem.

TheOREM 1.1. Letr: $\hat{\mathscr{S}}_{F} \rightarrow \hat{\mathscr{N}}_{F} / \operatorname{Ad}^{*} \mathscr{S}_{F}$ and $r_{1}: \hat{\mathscr{S}}_{F} \rightarrow \hat{C}_{\infty} / \operatorname{Ad}^{*} \mathscr{S}_{F}$ be the maps derived from restriction of representations. Let $\rho \in \hat{\mathscr{S}}_{F}$, $\rho_{1} \in \hat{C}_{\infty}, \sigma \in \hat{\hat{F}_{F}}$. Write $r(\rho)=\mathcal{O}_{1}, r_{1}(\rho)=\mathscr{O}_{1}$. Suppose $\rho_{1} \in \mathcal{O}_{1}$. Let $\hat{Q}_{1} \subseteq \hat{V}_{\infty}$ be the $\mathrm{Ad}^{*} \mathscr{S}_{F}$ orbit corresponding to $r_{1}(\rho)$. Let $\mathscr{S}_{\sigma}$ be the isotropy group of $\sigma$ under $\mathrm{Ad}^{*} \mathscr{S}_{F}$.

(i) $\mathscr{S}_{F}$ is type I. 
(ii) $\mathscr{S}_{F}$ is C.C.R. if and only if the split part of a maximal torus over $F$ of $\mathscr{S}$ is central in $\mathscr{L}$.

(iii) The Mackey obstruction to extending $\sigma$ from $\mathscr{N}_{F}$ to $\mathscr{S}_{\sigma}$ vanishes.

(iv) All representations $\rho$ of $\mathscr{S}_{F}$ are induced from finite dimensional representations of suitable subgroups. All representations $\rho_{1}$ of $C_{\infty}$ are induced from one-dimensional representations of suitable subgroups.

(v) The restriction of $\rho$ to $\mathscr{N}_{F}$ is multiplicity-free. The restriction of $\rho$ to $C_{\infty}$ is discretely decomposable and multiplicityfree. It is the direct sum of the representations $\operatorname{Ad}^{*} s\left(\rho_{1}\right), s \in \mathscr{S}_{F}$, each appearing once.

(vi) $\rho$ is C.C.R. if and only if $\hat{Q}_{1}$ is closed in $\hat{V}_{\infty}$.

(vii) Any representation $\rho^{\prime} \in r^{-1}(\mathcal{O})$ is of the form $\tau \otimes \rho$ wnere $\tau$ is a character of $\mathscr{S}_{F} / \mathscr{N}_{F}$. Moreover, $\tau \otimes \rho$ is equivalent to $\rho$ if and only if $\tau$ is trivial on $\mathscr{S}_{\psi} / \mathscr{N}_{\psi}$. Thus $r^{-1}(\mathcal{O}) \cong \hat{\mathscr{T}}_{F} / \mathscr{T}_{\sigma}{ }^{\perp} \cong \hat{\mathscr{T}}_{\sigma}$, where $\mathscr{T}_{F} \cong \mathscr{S}_{F} / \mathscr{N}_{F}, \mathscr{T}_{\sigma} \cong \mathscr{S}_{\sigma} / \mathscr{N}_{\sigma}$.

REMARKS. As a complement to (vi), we note that it follows from (v) and Proposition 1.1, that if $\rho$ is C.C.R. and $f \in C_{c}^{\infty}\left(C_{\infty}\right)$, then $\operatorname{tr} \rho(f)$ is given by the integral of the Fourier transform of $f$ over $\widetilde{O_{1}}$. We can say more. Even if $\rho$ is not C.C.R., $\rho(f)$ will be of trace class if (and only if) the intersection of $\tilde{O}_{1}$ with the support of $\hat{f}$ is closed. In this case the trace of $f$ is again by the orbital integral. It is interesting in this connection that if $r_{1}(\rho)=r_{1}\left(\rho^{\prime}\right)$ for two different representations $\rho$ and $\rho^{\prime}$, then evidently the traces of $\rho(f)$ and $\rho^{\prime}(f)$ will be the same, for $f \in C_{c}^{\infty}\left(C_{\infty}\right)$. Pukanszky [33] has observed a similar fact for real algebraic groups. Here, however, there is a simple explanation since $\rho$ and $\rho^{\prime}$ look so much alike. We observe also that the existence of many functions which are mapped into trace class operators, and the expected formula for their traces, even for nonC.C.R. representations, has recently been pointed out by C.C. Moore [25], Pukanszky [30] and others.

As the final point to our analysis, we try to fix a good "base point" for $r^{-1}(\mathscr{O})$ when $\mathscr{\mathscr { S }}$ is split. We will also comment on the problem when $\mathscr{S}$ is not split. For now, however, assume $\mathscr{S}$ is split.

Pick an $\mathrm{Ad}^{*} \mathscr{N}_{F}$ orbit $\mathscr{O} \subseteq \hat{N}_{F}$, and pick $\psi \in \mathcal{O}$. Let $\mathscr{S}_{\psi}$ be the isotropy group of $\psi$ under $\mathrm{Ad}^{*} \mathscr{S}_{F}$. By Proposition 1.3, for $\mathscr{S}$ split, we can find on algebraic subgroup $\mathscr{P} \subseteq \mathscr{N}$, such that $\mathscr{P}_{F}$ polarizes $\psi$ and is normalized by $\mathscr{S}_{\psi}$. Let $\psi^{\prime}$ be the linear character of $\mathscr{P}_{F}$ obtained by restricting $\psi^{\circ} \log$ to $\mathscr{P}_{F}$. Put $\sigma=\operatorname{ind}_{\mathscr{P}_{F}}^{\mathscr{F}_{F}} \psi^{\prime}$. We want to extend $\sigma$ to a representation $\sigma^{\prime}$ of $\mathscr{S}_{\sigma}=\mathscr{S}_{\psi} \cdot \mathscr{N}_{F}$ in a well-defined way. We do this as follows. Write $\mathscr{S}_{\psi}=\mathscr{T}_{\psi} \times_{s} \mathscr{N}_{\psi}$, with $\mathscr{T}_{\psi}$ the 
$F$-rational points of a maximal reductive subgroup over of $\mathscr{S}_{\psi}$, and $\mathscr{N}_{\psi}=\mathscr{S}_{\psi} \cap \mathscr{N}_{F}$. Then $\mathscr{S}_{\sigma}=\mathscr{S}_{\psi} \mathrm{X}_{s} \mathscr{N}_{F}$. Also $\mathscr{S}_{\psi} \cdot \mathscr{P}_{F}=\mathscr{T}_{\psi} \mathrm{X}_{s} \mathscr{P}_{F}$. Clearly $\mathrm{Ad}^{*} \mathscr{T}_{\psi}$ leaves $\psi^{\prime}$ on $\mathscr{P}_{F}$-invariant. Thus we may extend $\psi^{\prime}$ to a linear character $\varphi$ on $\mathscr{T}_{\psi} \mathbf{X}_{s} \mathscr{P}_{F}$ by letting $\varphi$ be trivial on $\mathscr{T}_{\psi}$. Then we will call the representation $\sigma^{\prime}$ of $\mathscr{T}_{\psi} \times_{s} \mathscr{N}_{F}$ induced from $\phi$ the standard extension of $\sigma$. The main point about $\sigma^{\prime}$ is that it does not depend on the various choices we have made. As we said, this is an analogue of Kostant's independence of polarization theorem.

PROPOSITION 1.4. $\varphi$ is independent of the choice of the reductive component $\mathscr{T}_{\psi}$ of $\mathscr{S}_{\psi}$. The representation $\sigma^{\prime}$ is independent of the choice of $\mathscr{P}$ subject to the conditions that $\mathscr{P}_{F}$ polarizes $\psi$ and is normalized by $\mathscr{S}_{\psi}$, and is independent of the choice of $\psi \in \mathcal{O}$.

Proof. If $\mathscr{T}_{\psi}^{\prime}$ is another maximal reductive subgroup of $\mathscr{S}_{\psi}$, then we know by [14] that $\mathscr{T}_{\psi}$ and $\mathscr{T}_{\psi}^{\prime}$ are conjugate in $\mathscr{S}_{\psi}$. If $\mathscr{T}_{\psi}^{\prime}=n^{-1} \mathscr{T}_{\psi} n$ for some $n \in \mathscr{N}_{\psi}$, then $\phi^{\prime}=\operatorname{Ad}^{*} n(\phi)$, where $\phi$ and $\varphi^{\prime}$ are the extensions of $\psi^{\prime}$ defined by using $\mathscr{T}_{\psi}$ and $\mathscr{T}_{\psi}^{\prime}$ respectively. But since $\phi$ is a linear character of $\mathscr{T}_{\psi} \times_{s} \mathscr{P}_{F}$ and $n \in \mathscr{N}_{\psi} \subseteq \mathscr{P}_{F}$, we see $\varphi^{\prime}=\varphi$.

If $\psi_{1}=\operatorname{Ad}^{*} n(\psi)$, for $n \in \mathscr{N}_{F}$, is another point in $\mathcal{O}$, then the isotropy group of $\psi_{1}$ is $n^{-1} \mathscr{S}_{\psi} n$, which has split component $n^{-1} \mathscr{T} \psi n$. If $\mathscr{P}_{F}$ polarizes $\psi$ and is normalized by $\mathscr{S}_{\psi}$, then $n^{-1} \mathscr{P}_{F} n$ polarizes $\operatorname{Ad}^{*} n(\psi)$ and is normalized by $n^{-1} \mathscr{S}_{\psi} n$. If $\phi$ is the extension of $\psi^{\prime}$ to $\mathscr{T}_{\psi} X_{S} \mathscr{P}_{F}$ defined above, then $\varphi_{1}(x)=\varphi\left(n X n^{-1}\right)$ for

$$
x \in n^{-1}\left(\mathscr{T}_{\psi} \underset{s}{\times} \mathscr{P}_{F}\right) n
$$

is the corresponding extension of $\psi_{1}^{\prime}\left(\psi_{1}^{\prime}=\psi_{1} \circ\right.$ log restricted to $\left.n^{-1} \mathscr{P}_{F} n\right)$ to $n^{-1}\left(\mathscr{T}_{\psi} \times \mathscr{P}_{F}\right) n$. Obviously $\varphi$ and $\varphi_{1}$ induce the same representation $\sigma^{\prime}$ of $\mathscr{S}_{\boldsymbol{\sigma}}$.

So we come to the main point, that $\sigma^{\prime}$ is independent of the choice of $\mathscr{\mathscr { P }}$. This is proved in the classic manner, by induction on the dimension of $\mathscr{S}_{\sigma}$. The group $\mathscr{N}_{\psi}$ obviously contains $\mathscr{Z}\left(\mathscr{N}_{F}\right)$, the center of $\mathscr{N}_{F}$. Let $\mathscr{H}$ be the largest algebraic subgroup of $\mathscr{Z}\left(\mathscr{N}_{F}\right)$ contained in the kernel of $\psi \circ \log$ on $\mathscr{N} \psi$. Then $\mathscr{H}$ is obviously contained in the kernel of $\sigma$, so by dividing out by it, if necessary, we may assume that $\mathscr{H}$ is trivial. Then $\mathscr{Z}\left(\mathscr{N}_{F}\right)$ is one-dimensional, and $\psi \circ \log$ is nontrivial on it.

Now let $\mathscr{F}$ be a two-dimensional normal subgroup of $\mathscr{S}_{a}$, such that $\mathscr{Z}\left(\mathscr{N}_{F}\right) \subseteq \mathscr{J} \subseteq \mathscr{N}_{F}$ and $\mathscr{I} / \mathscr{Z}\left(\mathscr{N}_{F}\right)$ is central in $\mathscr{N}_{F} / \mathscr{Z}\left(\mathscr{N}_{F}\right)$. Such a subgroup $\mathscr{I}$ exists because $\mathscr{S}_{\sigma}$ is split. Let $\mathscr{N}_{F}^{1}$ be the centralizer of $\mathscr{J}$ in $\mathscr{N}_{F}$. Then a simple calculation shows $\operatorname{dim} \mathscr{N}_{F}^{1}=$ 
$\operatorname{dim} \mathscr{N}_{F}-1$, and taking commutators defines a nondegenerate pairing from $\mathscr{F} / \mathscr{Z}\left(\mathscr{N}_{F}\right) \times \mathscr{N}_{F} / \mathscr{N}_{F}^{1}$ to $\mathscr{Z}\left(\mathscr{N}_{F}^{1}\right)$. By our construction, since $\psi \circ \log$ is nontrivial on $\mathscr{Z}\left(\mathscr{N}_{F}\right), \mathscr{N}_{F}^{1}$ will contain $\mathscr{N}_{\psi}$. Let $\psi^{1}$ be the restriction of $\psi$ to $N_{F}^{1}$, where $N_{F}^{1}=\log \mathscr{N}_{F}^{1} \subseteq \mathscr{N}_{F}$. Let $\sigma_{1}$ be the representation of $\mathscr{N}_{F}^{1}$ corresponding to $\operatorname{Ad}^{*} \mathscr{N}_{F}^{1}\left(\psi^{1}\right)$. Let $\sigma_{1}^{\prime}$ be the standard extension of $\sigma_{1}$ to $\mathscr{S}_{\psi} \cdot \mathscr{N}_{F}^{1}=\mathscr{T}_{\psi} \mathrm{X}_{s} \mathscr{N}_{F}^{1}$.

Let $\mathscr{P}_{F}^{1}$ be any algebraic subgroup of $\mathscr{N}_{F}^{1}$ which polarizes $\psi^{1}$ and is normalized by $\mathscr{S}_{\psi}$. Then necessarily $\mathscr{I} \subseteq \mathscr{P}^{1}$ since $\mathscr{J}$ is central in $\mathscr{N}_{F}^{1}$. It follows then that $\mathscr{P}_{F}^{1}$ polarizes $\psi$. Thus as $\sigma_{1}$ is obtained from inducing $\psi^{1} \circ \log$ on $\mathscr{P}_{F}^{1}$ up to $\mathscr{N}_{F}^{1}$, and $\sigma$ is obtained from inducing $\psi \circ$ log on $\mathscr{P}_{F}^{1}$ up to $\mathscr{N}_{F}$, it follows that $\sigma_{1}$ on $\mathscr{N}_{F}^{1}$ induced to $\mathscr{N}_{F}$ gives $\sigma$. Precisely the same chain of reasoning shows that $\sigma^{\prime}$ on $\mathscr{S}_{\sigma}$ is induced from $\sigma_{1}^{\prime}$ on $\mathscr{S}_{\psi} \cdot \mathscr{N}_{F}^{1}$. By hypothesis $\sigma_{1}^{\prime}$ and hence $\sigma^{\prime}$ is independent of the choice of $\mathscr{P}_{F}^{1} \subseteq \mathscr{N}_{F}^{1}$.

Now suppose $\mathscr{P}_{F}$ is a polarizing group for $\psi$ not contained in $\mathscr{N}_{F}^{1}$, and normalized by $\mathscr{S}_{\psi}$. Then necessarily $\mathscr{J} \cap \mathscr{P}_{F}=\mathscr{K}\left(\mathscr{N}_{F}\right)$, and $\mathscr{P}_{F}^{1}=\left(\mathscr{P}_{F} \cap \mathscr{N}_{F}^{1}\right) \cdot \mathscr{J}$ also polarizes $\psi$, and is normalized by $\mathscr{S}_{\psi}$. Let $\varphi$ be the extension of $\psi \circ \log$ from $\mathscr{P}_{F}$ to $\mathscr{T}_{\psi} \times_{s} \mathscr{P}_{F}$ which is trivial on $\mathscr{T}_{\psi}$ and let $\varphi^{1}$ be the extension, trivial on $\mathscr{T}_{\psi}$, of $\psi \circ \log$ from $\mathscr{P}_{F}^{1}$ to $\mathscr{T}_{\psi} \times_{s} \mathscr{P}_{F}^{1}$. Let $\mathscr{Q}=\mathscr{P}_{F} \cdot \mathscr{T}_{\text {. }}$ Then $\mathscr{P}_{F} \cong \mathscr{Q}$, and $\mathscr{P}_{F}^{1} \subseteq \mathbb{Q}$. To show $\varphi$ and $\varphi^{1}$ induce the same representation of $\mathscr{S}_{\sigma}$, it is enough to show they induce the same representation of $\mathscr{Q}$.

Let $\mathscr{Q}^{1}$ be the largest algebraic subgroup of $\mathscr{P}_{F} \cap \mathscr{P}_{F}^{1}$ contained in the kernel of $\psi \circ \log$. Then $\mathscr{Q}^{1}$ is normalized by $\mathscr{T} \psi$ and is normal in $\mathbb{Q}$. The group $\mathbb{Q}^{\prime}=\mathscr{Q} / \mathbb{Q}^{1}$ is a three-dimensional Heisenberg group. The characters $\varphi$ and $\varphi_{1}$ factor to $\mathscr{T}_{\psi} X_{s} \mathscr{P}_{F} / \mathscr{Q}^{1}$ and $\mathscr{T}_{\psi} \mathrm{X}_{s} \mathscr{P}_{F}^{1} / \mathscr{Q}^{1}$ respectively. Similarly the representations of $\mathscr{T}_{\psi} \mathrm{X}_{s} \mathscr{Q}$ induced from $\varphi$ and $\phi^{1}$ both factor to representations of $\mathscr{T} \psi \times_{s} \mathscr{Q} \mid \mathscr{Q}^{1}$. Furthermore, if $\mathscr{R} \subseteq \mathscr{T}_{\psi}$ is the kernel of the adjoint action of $\mathscr{T}_{\psi}$ on $\mathscr{Q} \mid \mathscr{Q}^{1}$, then $\mathscr{R}$ is likewise in the kernel of the representations of $\mathscr{T}_{\psi} X_{s} \mathscr{Q}$ induced from both $\varphi$ and $\varphi^{1}$. Thus to prove the proposition, it is enough to consider the case when $\mathscr{N}_{F}$ is the threedimensional Heisenberg group and $\mathscr{T}_{\psi}$ acts effectively on $\mathscr{N}_{F}$. Moreover, it is completely clear that we need only worry when $\mathscr{T}_{\psi}$ is nontrivial. We now concentrate on this case.

Let us change notation slightly. Let $\mathscr{H}$ denote the threedimensional Heisenberg group. Let its Lie algebra be $H$. Let $\mathscr{Z}$. be the one-dimensional center of $\mathscr{H}$, and let $\psi$ be a character on $\mathscr{Z}$. Let $F^{x}$, the multiplicative group of $F$, act on $\mathscr{H}$ by an action $\mathrm{Ad}$, as follows. Write $H=V_{1} \oplus V_{2} \oplus \log \mathscr{\not}$, where $V_{1}$ and $V_{2}$ are onedimensional subspaces. Take $x \in F^{x}$. For $v \in V_{1}$, put $\operatorname{Ad} x(v)=x v$; for $v \in V_{2}$, put $\operatorname{Ad}(x)(v)=x^{-1} v$. For $x \in \log \not{z}$, put $\operatorname{Ad} x(z)=z$. Then Ad is in fact an action by automorphisms, and using exp and log, we may transfer Ad to an action of $F^{x}$ on $\mathscr{H}$ by automorphisms. 
Form the corresponding semidirect product $G=F^{x} X_{s} \mathscr{H}$. Then it is easy to see that $G$ is in fact a split algebraic solvable group over $F$, and that the action of $F^{x}$ on $\mathscr{H}$ fixes $\psi$ and hence fixes the representation of $\mathscr{H}$ corresponding to $\psi$ by Lemma 1.2. It is not hard to see also that $G$ is maximal with respect to these properties in the following sense. With the notation of the previous paragraph, if $\mathscr{T}_{\psi}$ acts effectively on $\mathscr{Q} / \mathbb{Q}^{1}$, then there is an injective homomorphism $\mathscr{T}_{\psi} \times_{s} \mathscr{Q} / \mathbb{Q}^{1} \rightarrow G$, such that the image of $\mathscr{T}_{\psi}$ is in $F^{x}$. Therefore, in proving our result, we need consider only $G$.

There are precisely two two-dimensional subgroups, $\mathscr{C}^{1}$ and $\mathscr{H}^{2}$, of $\mathscr{H}$ which are normalized by $F^{x}$. Each $\mathscr{H}^{i}$ is the image under the exponential map of $V_{i} \oplus \log \mathscr{z}$. Put $G^{i}=F^{x} X_{s} \mathscr{H}^{i}$. On $G^{i}$ there is a unique character $\varphi^{i}$ which is trivial on $F^{x}$ and agrees with $\psi$ on $\mathscr{Z}$. Observe $\varphi^{i}$ must be trivial on $\exp V_{i}$ We must show the representations of $G$ induced from the $\phi^{i}$ on $G^{i}$ are equivalent, and then we will be done.

Recall the explicit definition of $\operatorname{ind}_{G}^{G} i \varphi^{i}$. See [7] for details. Let $\delta_{i}$ be the modular function on $G^{i}$. That is, if $d^{i} g$ is a right Haar measure on $G^{i}, d^{i}\left(g_{0} g\right)=\delta_{i}\left(g_{0}\right) d^{i} g$ for any $g_{0} \in G^{i}$. Let $Y^{i}$ be the space of locally constant functions $f$ on $G$ with compact support modulo $G^{i}$, and such that for $g \in G^{i}, y \in G, f(g y)=\varphi^{i}(g) \delta_{i}^{1 / 2}(g) f(y)$. G acts on $Y^{i}$ to the right, by translation. There is an essentially unique $G$ invariant inner product on $Y^{i}$ and the completion of $Y^{i}$ with respect to this inner product yields an Hilbert space on which $G$ acts unitarily. This is the representation $\operatorname{ind}_{G i}^{G} \varphi^{i}$.

To show these representations are equivalent, it is enough to define a $G$-invariant nontrivial Hermitian (linear in one variable, conjugate linear in the other) pairing between them, and to do this it is enough to define a nontrivial Hermitian pairing between the $Y^{i}$. See the discussion in [20], pp. 23-30. To define this pairing, take $f_{i} \in Y^{i}$ and consider the product $f_{1} \bar{f}_{2}$ where - indicates complex conjugate. Since $f_{i}$ has compact support modulo $G^{i}, f_{1} \bar{f}_{2}$ has compact support modulo $G^{1} \cap G^{2}=F^{x} \times \mathscr{Z}$. Moreover, if $g \in G^{1} \cap G^{2}$, and $y \in G$, then $f_{1} \bar{f}_{2}(g y)=\delta_{1}^{1 / 2}(g) \delta_{2}^{1 / 2}(g) f_{1} \bar{f}_{2}(y)$. Moreover, we note that since $G^{1} \cap G^{2}$ is abelian, it is unimodular. Since $G$ is unimodular also, and $G=G^{1} \cdot G^{2}$, we have $\delta_{1}(g) \delta_{2}(g)=1$ for $g \in G^{1} \cap G^{2}$. Thus $f_{1} \bar{f}_{2}$ is a function on $X=G^{1} \cap G^{2} \backslash G$. Again, since $G^{1} \cap G^{2}$ and $G$ are unimodular, there is a $G$-invariant measure $d x$ on $X$, unique up to multiples ([36]). Define $\left\langle f_{1}, f_{2}\right\rangle=\int_{X} f_{1} \bar{f}_{2} d x$. This is our pairing. It is easy to see $\langle$,$\rangle is G$-invariant, because $d x$ is $G$-invariant. To show $\langle$,$\rangle is$ nontrivial is also not hard. Choose a sufficiently small neighborhood $U$ of the identity and suppose $f_{i}$ vanishes outside $G^{i} U$ and is identically one on $U$. We leave the details to the reader. This concludes Pro- 


\section{position 1.4.}

To establish the analogue of Proposition 1.4 for nonsplit groups, one would use Proposition 1.3 plus the demonstration that the Mackey obstruction vanishes. For brevity, we adopt the notation of that demonstration. Our problem is to define a "canonical" extension of the representation $\sigma^{1}$ of $G^{3}$ to $\mathscr{T}_{\psi} \times_{s} G^{3}$. Since the restriction of $\sigma^{1}$ to $G^{1}$ is a multiple of a linear character of $G^{1}$, the problem essentially reduces to a problem over the residue-class field of $F$. In this context we have a candidate for a canonical extension, definable via the Weil representation for finite fields. To see whether this candidate was the right one, one would have to investigate it in detail, then verify it has the proper independence properties. One should also check for consistency with the above definition and with the orbital parametrization for real groups.

II. Representations defined by solvmanifolds: Reduction of the problem. In this section $\mathscr{S}$ will denote a solvable Lie group and $\Gamma$ a discrete subgroup of $\mathscr{S}$ such that the quotient is compact. Then $\mathscr{S}$ is unimodular and $\mathscr{S} / \Gamma$ carries a unique probability measure $d \mu$ which is invariant under left translation by $\mathscr{S}$. Thus if $L^{2}(\mathscr{S} / \Gamma)$ is the Hilbert space of square integrable functions on $\mathscr{S} / \Gamma$ with respect to $d \mu$, then we have an action of $\mathscr{S}$ on $L^{2}(\mathscr{S} \mid \Gamma)$ by unitary operators. If this action is denoted by $U$, and $f \in L^{2}(\mathscr{S} \mid \Gamma)$, we have the formula $U(s) f(x)=f_{s^{-1}}(x)=f\left(s^{-1} x\right)$, for $s \in \mathscr{S}, x \in \mathscr{S} \mid \Gamma$. We will consider the problem of decomposing $L^{2}(\mathscr{S} \mid \Gamma)$ into its irreducible constituents. In the case $\mathscr{S}$ is actually nilpotent, this problem was solved in [16] and [34]. We begin with an elementary general discussion in order to put the results of [16] and [34] in perspective, to make clear in what measure those results are general and in what measure they require special properties of nilpotent groups.

First let us reformulate the multiplicity result of [16] and [34]. Let $\mathscr{N}$ be a nilpotent Lie group, and let $\Gamma \subseteq \mathscr{N}$ still be a discrete subgroup such that $\mathscr{N} / \Gamma$ is compact. Let $\rho$ be an irreducible unitary representation of $\mathscr{T}$. Then $\rho$ corresponds by Kirillov theory to a certain orbit $\mathcal{O}$ of the co-adjoint action of $\mathscr{N}$ in the dual of the Lie algebra of $\mathscr{N}$. In [16] and [34] the multiplicity of $\rho$ in $L^{2}(\mathscr{N} / \Gamma)$ is computed in terms of the number of $\Gamma$ orbits among certain "integral points" in a homogeneous space associated to $O$. The precise formulation is directly tied into the Kirillov theory for $\mathscr{N}$ and is slightly complicated. I believe, however, that the connection of this result with Kirillov theory is somewhat superficial and even, perhaps, in some ways misleading (although of course for purposes of taking a unified approach toward nilpotent groups, such a formulation is desirable). As was rather elliptically noted in [16], the result may 
be restated more simply in the following way. Recall (see [24]) that $\Gamma$ gives rise to a $Q$-structure on $\mathscr{N}(Q=$ rational numbers $)$, so that $\mathscr{N}$ is the real points of a unipotent algebraic group over $Q$. Let $\mathscr{H}$ be a rational algebraic subgroup of $\mathscr{N}$ with respect to this $Q$-structure, and let $\psi$ be a character of $\mathscr{H}$. Suppose that $\rho=$ $\operatorname{ind}_{\mathscr{F}} \mathscr{\psi} \psi$. Then the multiplicity of $\rho$ in $L^{2}(\mathscr{N} / \Gamma)$ is equal to the number of double cosets $\mathscr{H} m \Gamma, m \in \mathscr{N}$, such that $\mathscr{H} / \mathscr{H} \cap m \Gamma m^{-1}$ is compact and $\psi$ is trivial on $\mathscr{H} \cap m \Gamma m^{-1}$.

Compare this with the analogous statement for finite groups, due to Mackey [23]. Suppose $G$ is a finite group, and $H_{1}$ and $H_{2}$ are two subgroups. Let $\rho$ be an irreducible representation of $G$ and suppose $\rho$ is induced from some linear character $\psi$ of $H_{1}$. Then the multiplicity of $\rho$ in $L^{2}\left(G / H_{2}\right)$ is equal to the number of double cosets $H_{1} g H_{2}$ such that $\psi$ is trivial on $H_{1} \cap g H_{2} g^{-1}$. The resemblance between the statements is evident. To go from the context of finite groups to that of nilpotent Lie groups we must first restrict our attention to cosets $\mathscr{H} m \Gamma$ which are closed. (The condition that $\mathscr{H} \cap m \Gamma m^{-1}$ be compact is equivalent to asking that $\mathscr{H} m \Gamma$ be closed, by a standard argument. See [28].) Let us call such cosets "good" double cosets. Second, we must only consider rational subgroups $\mathscr{H}$ (or at most, subgroups conjugate in $\mathscr{N}$ to rational subgroups). This restriction functions to insure the existence of sufficiently many good $(\mathscr{H}, \Gamma$ ) double cosets. That is, examples show if $\rho$ is induced from $\psi$ on $\mathscr{H}$, then the number of good double cosets $\mathscr{H} m \Gamma$ such that $\psi$ is trivial on $\mathscr{H} \cap m \Gamma m^{-1}$ tends to underestimate the multiplicity of $\rho$ in $L^{2}(\mathscr{N} / \Gamma)$. For instance, if $\mathscr{H}$ is not rational, but is normal, then there are no good ( $\mathscr{H}, \Gamma$ ) double cosets, but $\rho$ may appear with positive multiplicity. This already happens in the Heisenberg group. In the first few propositions, we look at the method of determining multiplicity by counting double cosets. We give examples where it works, and show how it tends to give lower bounds for multiplicity.

Let $G$ be a locally compact separable unimodular group, and let $\Gamma$ be a discrete subgroup such that $G / \Gamma$ is compact. Then $G / \Gamma$ carries a unique $G$-invariant probability measure $d x$, and we may consider the representation of $G$ on $L^{2}(G / \Gamma)=L^{2}(G / \Gamma, d x)$ derived from the left action of $G$ on $G / \Gamma$. Of course, in terms of induced representations, this representation is just $\operatorname{ind}_{\Gamma}^{G} 1$, where the 1 here denotes the trivial representation of $\Gamma$. Let $\rho$ be an irreducible representation of $G$. We want to determine whether $\rho$ occurs in $L^{2}(G / \Gamma)$ and if so, how often. We will suppose there is a closed unimodular subgroup $H \subseteq G$ such that $H \cap \Gamma$ is compact, and that there is a representation $\sigma$ of $H$ which induces $\rho$. Then this first result is an example of what one can say. 
Proposition 2.1. (i) Suppose $H$ is normal in $G$. Then all $(H, \Gamma)$ double cosets are good. For a given double coset $H g \Gamma$, let $m_{g}$ be the multiplicity of $\sigma$ in $L^{2}\left(H / H \cap g \Gamma g^{-1}\right)$. Then the number of double cosets such that $m_{g}>0$ is finite, and the multiplicity of $\rho$ in $L^{2}(G / \Gamma)$ is exactly equal to $\sum_{g \in H \backslash G / \Gamma} m_{g}$.

(ii) Suppose $H=H_{0} \subseteq H_{1} \subseteq \cdots \leqq H_{l}=G$, where $H_{i} / H_{i} \cap \Gamma$ is compact and $H_{i}$ is normal in $H_{i+1}$. Then the multiplicity of $\rho$ in $L^{2}(G / \Gamma)$ is at least equal to the multiplicity of $\sigma$ in $L^{2}(H / H \cap \Gamma)$.

Proof. The proof is very simple. Part (i) is a translation of part of Proposition 1 of [16], and part (ii) follows easily by induction on $l$.

We recall the language necessary for translation of Proposition 1 of [16] to the present context. As described in part I, we have an action $\mathrm{Ad}^{*} G$ of $G$ on $\hat{H}$. We say that two $\mathrm{Ad}^{*} G$ orbits $O_{1}$ and $\mathrm{O}_{2}$ in $\hat{H}$ are equivalent if their closures are equal. An equivalence class of orbits is called a quasiorbit. We denote by $\hat{H} / \mathrm{Ad}^{*} G$ the space of quasiorbits endowed with the quotient topology from $\hat{H}$. We call $\hat{H} / \mathrm{Ad}^{*} G$ the relative primitive ideal space of $H$ in $G$.

We may define a continuous map $r: \hat{G} \rightarrow \hat{H} / \mathrm{Ad}^{*} G$. If $\rho$ is an irreducible representation of $G$ corresponding to some point $x \in \hat{G}$, the restriction of $\rho$ to $H$ will define a closed subset of $\hat{H}$ which will be the closure of an $\mathrm{Ad}^{*} G$ orbit and which therefore defines a a point in $\hat{H} / \mathrm{Ad}^{*} G$. This point is $r(x)$. By abuse of notation, we also write $r(\rho)$. Note that we have already encountered $r$, for the special case of a solvable $p$-adic group and its unipotent radical, in the context of Theorem 1.1 of part $I$.

Now if $\rho=\operatorname{ind}_{H}^{G} \sigma$, then $r(\rho)$ is the quasiorbit determined by $\sigma$. Consider $L^{2}(H / H \cap \Gamma)$. The representations of $H$ occurring here define a discrete subset of $\hat{H}$, and this subset is clearly invariant under $\mathrm{Ad}^{*} \Gamma$, and so is a union of $\mathrm{Ad}^{*} \Gamma$ orbits. Any two representations of $H$ which are in the same $\operatorname{Ad}^{*} \Gamma$ orbit clearly occur in $L^{2}(H / H \cap \Gamma)$ with the same multiplicity. By Proposition 1 of [16], $\rho$ can occur in $L^{2}(G / \Gamma)$ only if $r(\rho)=\mathrm{Ad}^{*} G(\sigma)=\mathrm{Ad}^{*} G\left(\sigma_{1}\right)$ for some $\sigma_{1}$ occurring in $L^{2}(H / H \cap \Gamma)$. If this happens, then $\rho$ does occur in $L^{2}(G / \Gamma)$ and with multiplicity given by $\Sigma n_{\alpha}$, where $\alpha$ runs over the $\mathrm{Ad}^{*} \Gamma$ orbits in $r(\rho)$ and $n_{\alpha}$ is the common multiplicity with which the representation in $\alpha$ occurs in $L^{2}(G / \Gamma)$. Of course $n_{\alpha}$ is nonzero for only finitely many $\alpha$.

Now it is clear that $\sigma$ occurs in $L^{2}\left(H / H \cap g \Gamma g^{-1}\right)$ if and only if $\mathrm{Ad}^{*} g(\sigma)$ occurs in $L^{2}(H / H \cap \Gamma)$, and with the same multiplicity. Since the isotropy group of $\sigma$ under $\operatorname{Ad}^{*} G$ is precisely $H$ (since $\sigma$ induces an irreducible representation of $G$ ), there is a one-to-one correspondence between $\mathrm{Ad}^{*} \Gamma$ orbits in $r(\rho)$ and $(N, \Gamma)$ double cosets, given by associating $N g \Gamma$ and $\mathrm{Ad}^{*} \Gamma\left(\mathrm{Ad}^{*} g(\sigma)\right)=\alpha(g)$. From our observation 
just above, we see that $m_{g}=n_{\alpha(g)}$. This proves the first statement of the proposition.

The second statement now follows from the first. Suppose $H=$ $H_{0} \subseteq H_{1} \subseteq \cdots \subseteq H_{l}=G$, where $H_{i} / H_{i} \cap \Gamma$ is compact and $H_{i}$ is normal in $H_{i+1}$. Put $\rho_{i}=\operatorname{ind}_{H}^{H_{i}} \sigma$. Then for $i<j, \rho_{j}=\operatorname{ind}_{H_{i}}^{H_{j}} \rho_{j}$. Since $H=$ $H_{0}$ is normal in $H_{1}$, we see from the first part that the multiplicity of $\rho_{1}$ in $L^{2}\left(H_{1} / H_{1} \cap \Gamma\right)$ is at least equal to the multiplicity of $\rho_{0}=\sigma$ in $L^{2}(H / H \cap \Gamma)$. Similarly, the multiplicity of $\rho_{2}$ in $L^{2}\left(H_{2} / H_{2} \cap \Gamma\right)$ is at least equal to the multiplicity of $\rho_{1}$ in $L^{2}\left(H_{1} / H_{1} \cap \Gamma\right)$. Following this reasoning on up the chain of $H_{i}$ 's, we obtain our result.

REMARKs. (a) Part (ii) of the proposition applies in particular to nilpotent Lie groups and generalizes the positive part of the occurrence criterion of [34]. It is perhaps a matter of some technical interest that we obtain here and elsewhere lower bounds for multiplicities. Most estimates in the literature are upper bounds. See [11], [23], and [24].

(b) Each of the two parts of the proposition is a partial generalization of the following comprehensive result on finite groups, due to Mackey [23]. If $G$ is finite, then the multiplicity of $\rho$ in $L^{2}(G / \Gamma)$ is $\sum_{g \in H \backslash G \mid \Gamma} m_{g}$. Thus when $G$ is finite, there is no need to take $H$ normal as in part (i), and one may consider all $(H, \Gamma)$ double cosets simultaneously instead of considering only one double coset, as in part (ii).

(c) The barrier to extending part (ii) to a statement about all good $(H, \Gamma)$ double cosets is the possibility that, even though $H g \Gamma$ is good double coset, $H_{i} g \Gamma$ might not be good for some $H_{i}$. A nilpotent Lie group $\mathscr{N}$ has the property that whenever $\mathscr{H} \subseteq \mathscr{N}$ is a subgroup such that $\mathscr{H} / \mathscr{H} \cap \Gamma$ is compact, then also $\mathscr{H}_{1} \mid \mathscr{H}_{1} \cap \Gamma$ is compact if $\mathscr{H}_{1}$ is the normalizer of $\mathscr{H}$ in $\mathscr{N}$. This fact derives from the fact that such an $\mathscr{N}$ containing discrete $\Gamma$ with compact quotient actually has the structure of an algebraic group defined over $Q$, the rational numbers. We may expect also for compact arithmetically defined homogeneous spaces, and arithmetically defined solvmanifolds in particular, that an extension of part (ii) of the proposition, involving all $(H, \Gamma)$ double cosets, will hold, In our present general context, we must be content with observing that if the chain connecting $H$ to $G$ contains only one intermediate group, that is, if $H=H_{0} \subseteq H_{1} \subseteq H_{2}=G$, with $H_{i} / \Gamma$ compact and $H_{i}$ normal in $H_{i+1}$, then all $\left(H_{1}, \Gamma\right)$ double cosets are good, and we obtain the following corollary.

CoRollary 1. Suppose that in part (ii) of Proposition 2.1 we have $l=2$. For each good double coset $H g \Gamma$, let $m_{g}$ denote the mul- 
tiplicity of $\sigma$ in $L^{2}\left(H / H \cap g \Gamma g^{-1}\right)$. Then the multiplicity of $\rho$ in $L^{2}(G / L)$ is at least equal to the sum of the $m_{g}$ over all good $(H, \Gamma)$ double cosets.

(d) It seems worthwhile to state explicitly the specialization of the proposition when $\sigma=\psi$ is a one-dimensional charater of $H$, since this situation is the easiest to deal with and occurs often in practice. So suppose $\rho=\operatorname{ind}_{H}^{G} \psi$. Notice that a linear character occurs in $L^{2}(G / \Gamma)$ if and only if it is trivial on $\Gamma$, and that then it occurs exactly once.

CoROLlaRy 2. (i) If $H$ is normal, then the multiplicity of $\rho$ in $L^{2}(G / \Gamma)$ is the number of double cosets $H g \Gamma$ such that $\psi$ is trivial on $H \cap g \Gamma g^{-1}$.

(ii) If $H \subseteq H_{0} \subseteq H_{1} \subseteq \cdots \subseteq H_{l}=G$, where $H_{i} / H_{i} \cap \Gamma$ is compact and $H_{i}$ is normal in $H_{i+1}$, and if $\psi$ is trivial on $H \cap \Gamma$, then $\rho$ occurs in $L^{2}(G / \Gamma)$.

We would like to point out that this corollary, simple minded as it is, already gives us enough information to decompose $L^{2}(G / \Gamma)$ when $G$ is metabelian. This already includes some interesting examples, notably various $a x+b$ groups.

Proposition 2.2. Retain the above notation. Suppose $N \subseteq G$ is a normal subgroup such that $N$ and $G / N$ are abelian, and $N / \Gamma$ is compact. Then every representation occurring in $L^{2}(G / \Gamma)$ is induced from a linear character $\psi_{1}$ of a group $N_{1}$, such that $N \cong N_{1}$, and $N_{1} / \Gamma \cap N_{1}$ is compact. (Note then that $N_{1}$ is normal.)

Proof. Since $N$ is abelian, $L^{2}(N / N \cap \Gamma)$ just decomposes into onedimensional spaces corresponding to the characters of $N / N \cap \Gamma$. For any given character $\psi$ on $N / N \cap \Gamma$, let $H$ be the isotropy group of $\psi$ under $\operatorname{Ad}^{*} G$ acting on $\hat{N}$. Since $H \supseteqq N, H$ is normal in $G$, and since the $\operatorname{Ad}^{*} \Gamma$ orbit of $\psi$ in $\hat{N}$ is discrete, since it belongs to the annihilator of $N \cap \Gamma, H / H \cap \Gamma$ will be compact, by a classical result [28].

Now in $L^{2}(H / H \cap \Gamma)$, the space of functions transforming by $N$ according to $\psi$ is invariant, and it is clearly naturally unitarily equivalent to the representation $\sigma$ of $H$ induced from $Y=(H \cap \Gamma) \cdot N$ by the linear character $\psi^{\prime}$ which agrees with $\psi$ on $N$ and is trivial on $\Gamma$. This extension $\psi^{\prime}$ of $\psi$ from $N$ to $Y$ exists because $Y / \operatorname{ker} \psi$ is isomorphic to $(N / \operatorname{ker} \psi) \times((H \cap \Gamma) / \operatorname{ker} \psi)$ (direct product) since $\Gamma \cap N \subseteq \operatorname{ker} \psi$.

Next we see that $Y$ is normal in $H$. Let $H^{\prime}$ be the isotropy group of $\psi^{\prime}$ under the action $\operatorname{Ad}^{*} H$ on $\hat{Y}$. Then clearly if $\sigma^{\prime}=\operatorname{ind}{ }_{Y}^{H^{\prime}} \psi^{\prime}$, then $\sigma^{\prime}$ induced up to $H$ yields $\sigma$, by transitivity of induced repre- 
sentations. Now, however, we see that $\sigma^{\prime}$ will in fact be trivial on $X=(H \cap \Gamma) \cdot \operatorname{ker} \psi$ because $\psi^{\prime}$ is trivial on $X$, and the kernel of $\psi^{\prime}$ is normal in $H^{\prime}$ by definition of $H^{\prime}$. Thus we may regard $\sigma^{\prime}$ as a representation of the factor group $H^{\prime} / X$. As such, it is the representation induced from $\psi^{\prime}$ factored to $Y / X$. Since $H / N$ is abelian, and since $Y / X$ is central in $H^{\prime} / X$, again by definition of $H^{\prime}$, we see that $H^{\prime} / X$ must be a compact two-step nilpotent group. It follows easily (see [17]) that all representations of $H^{\prime} / X$ are induced from linear characters of open subgroups. Lifting this information to $H^{\prime}$, we see that $\sigma^{\prime}$ decomposes into a direct sum of representations induced from linear characters of open subgroups of $H^{\prime}$.

Now consider a representation $\rho$ of $G$ occurring in $L^{2}(G / \Gamma)$. Then, according to [16], $\rho$ lies over $\operatorname{Ad}^{*} G(\psi) \subseteq \hat{N}$ for some character $\psi$ of $N / N \cap \Gamma$. If $H$ is as above, the isotropy group of $\psi$ under $\operatorname{Ad}^{*} G$, then $\rho$ will lie over a certain $\mathrm{Ad}^{*} G$ orbit in $\hat{H}$ and this orbit must contain representations occurring in $L^{2}(H / H \cap \Gamma)$. Moreover if $\sigma \in \hat{H}$ is such that $\sigma$ occurs in $L^{2}(H / H \cap \Gamma)$ and $\rho$ lies over $\mathrm{Ad}^{*} G(\sigma)$, then it follows from Mackey's theory, as explained in part $I$, that $\rho=$ $\operatorname{ind}_{H}^{G} \sigma$. But since we have seen above that $\sigma$ is induced from a linear character of a subgroup of $H$ containing $(H \cap \Gamma) \cdot N$, the proposition is established.

Before ending this general discussion, we make two further remarks. First, the normality restrictions in Proposition 2.1 essentially serve to ensure that certain formally definable intertwining operators make good sense. This has not been brought out explicitly because, in fact, when dealing with normal subgroups, one can avoid the problem of intertwining operators entirely. Second, aside from the normality restrictions, I hope it has become clear that the main obstacle to developing a general multiplicity result after the result of [16] and [34] is the possible lack sufficiently many "good" double cosets. Both these points will come up in our analysis for the case of solvable Lie groups.

We now turn to this case. Let $\mathscr{S}$ be our solvable Lie group, and let $\Gamma \subseteq \mathscr{S}$ be a discrete subgroup such that $\mathscr{S} / \Gamma$ is compact. We do not assume that $\mathscr{S}$ is connected. Instead we assume that if $\mathscr{S}^{0}$ is the connected component of the identity of $\mathscr{S}$, and if $\mathscr{N}$ is the nilradical of $\mathscr{S}^{0}$, then $\mathscr{S}^{0}$ is open in $\mathscr{S}$, (this may be taken as part of the definition of Lie group) and $\mathscr{S} / \mathscr{N}$ is abelian. We also take $\mathscr{N}$ to be simply connected. Then it follows that $\mathscr{S}$ may be embedded as a closed subgroup of a connected solvable Lie group (see [26]). Also it is clear that any closed subgroup of $\mathscr{S}$ containing $\mathscr{N}$ is normal in $\mathscr{S}$, and satisfies the same conditions. Also, if $\mathscr{S}$ is connected, it automatically satisfies our conditions. Note, too that $\mathscr{S}^{0} / \mathscr{S}^{0} \cap \Gamma$ will be compact. Furthermore, it was shown 
in [27] that $\mathscr{N} \Gamma$ is closed. That is, if $\Delta=\mathscr{N} \cap \Gamma$, then $\mathscr{N} / \Delta$ is compact.

Choose $\sigma \in \hat{\mathscr{N}}$ which occurs in $L^{2}(\mathscr{N} / \Delta)$. We will try to find the components of $L^{2}(\mathscr{S} \mid \Gamma)$ which lie above $\operatorname{Ad}^{*} \mathscr{S}(\sigma) \subseteq \hat{\mathscr{N}}$ Let $\mathscr{R} \cong \mathscr{S}$ be the isotropy group of $\sigma$ under $\mathrm{Ad}^{*} \mathscr{S}$. Then $\mathscr{N} \cong \mathscr{R}$ and $\mathscr{R} \mid \mathscr{R} \cap \Gamma$ is compact and the subspace of $L^{2}(\mathscr{R} \mid \mathscr{R} \cap \Gamma)$ which transforms under $\mathscr{N}$ according to $\sigma$ is invariant under $\mathscr{R}$. Thus, if we can decompose this subspace, we can in principle use Proposition 2.1 to compute the components of $L^{2}(\mathscr{S} \mid \Gamma)$ lying above $\operatorname{Ad}^{*} \mathscr{S}(\sigma)$, since $\mathscr{R}$ is normal in $\mathscr{S}$, and by Mackey's theory all such components are induced from $\mathscr{R}$. Thus we are essentially reduced to the case $\mathscr{R}=\mathscr{S}$, so that $\mathrm{Ad}^{*} \mathscr{S}(\sigma)=\sigma$, and we now assume we are in this situation.

We would like to reduce still further, and be able to assert that $\sigma$ actually extends to an irreducible representation of $\mathscr{S}$. We show that by again restricting our attention to a suitable subgroup, we can accomplish this also. We could give a special argument based on explicit realizations of representions of $\mathscr{S}$, but at the moment a more general argument is convenient.

Let $\mathscr{R} \subseteq \mathscr{S}$ now denote a subgroup of $\mathscr{S}$ containing $\mathscr{N}$ such that

(i) $\mathscr{R}$ is connected,

(ii) $\mathscr{R} / \mathscr{R} \cap \Gamma$ is compact, and

(iii) the representation $\sigma$ extends to a representation $\rho$ of $\mathscr{R}$ in the sense that $\rho$ restricted to $\mathscr{N}$ is irreducible and equivalent to $\sigma$.

Suppose further that $\mathscr{R}$ is maximal with respect to the above three properties. It is clear that maximal $\mathscr{R}$ 's exist, since all increasing sequences of connected subgroups of $\mathscr{S}$ are finite. Now let $\mathscr{R}^{\prime}$ be the isotropy group of $\rho \in \mathscr{R}$ under the action of $\mathrm{Ad}^{*} \mathscr{S}$. I claim that the identity component of $\mathscr{R}^{\prime}$ is precisely $\mathscr{R}$. For, as usual $\mathscr{R}^{\prime} \mid \mathscr{R}^{\prime} \cap \Gamma$ is compact. Thus if $\mathscr{R}$ is not the whole identity component of $\mathscr{R}^{\prime}$, then we may find a connected group $\mathscr{R}^{\prime \prime} \leqq \mathscr{R}^{\prime}$ such that $\mathscr{R}^{\prime \prime} \mid \mathscr{R}^{\prime \prime} \cap \Gamma$ is compact, $\mathscr{R} \subseteq \mathscr{R}^{\prime \prime}$, and $\operatorname{dim} \mathscr{R}^{\prime \prime}=\operatorname{dim} \mathscr{R}+1$. Of course $\rho \in \hat{R}$ will be invariant under $\mathrm{Ad}^{*} \mathscr{R}^{\prime \prime}$. But in such a situation, since $\mathscr{R}^{\prime \prime}$ is generated by $\mathscr{R}$ and a single one parameter subgroup, $\rho$ may be extended to an irreducible representation of $\mathscr{R}^{\prime \prime}$, by [5]. This contradicts the maximality of $\mathscr{R}$, and so we conclude that, indeed, $\mathscr{R}$ is the connected component of $\mathscr{R}^{\prime}$. Now simply let $\mathscr{R}_{1}$ denote a maximal subgroup of $\mathscr{R}^{\prime}$ containing $\mathscr{R}$ such that $\rho$ extends to a representation $\rho_{1}$ of $\mathscr{R}_{1}$. Then $\mathscr{R}_{1}$ will be a closed subgroup of $\mathscr{R}^{\prime}$, hence of $\mathscr{S}$, and an argument like the above, shows that the isotropy group of $\rho_{1} \in \mathscr{R}_{1}$ under $\mathrm{Ad}^{*} \mathscr{S}$ is precisely $\mathscr{R}_{1}$ itself.

Since $\sigma$ extends to $\rho_{1}$ on $\mathscr{R}_{1}$, it follows by Mackey's theory, as explained in part $\mathrm{I}$, that any representation of $\mathscr{R}_{1}$ lying above $\sigma$ 
on $\mathscr{N}$ is of the form $\rho_{1} \otimes \psi$, where $\psi$ is a linear character of $\mathscr{R}_{1}$, trivial on $\mathscr{N}$. Now let $\tau$ be a representation of $\mathscr{S}$ lying over $\sigma$ on $\mathscr{N}$. Then the restriction of $\tau$ to $\mathscr{R}_{1}$ is clearly a direct integral of representations lying above $\sigma$, so that it is a direct integral over representations of the form $\rho_{1} \otimes \psi$, where $\psi$ varies in $\left(\mathscr{R}_{1} / \mathscr{N}\right)^{\wedge}$. Since the commutator group of $\mathscr{S}$ is contained in $\mathscr{N}$, the isotropy group under $\operatorname{Ad}^{*} \mathscr{S}$ of $\rho_{1} \otimes \psi$ must be $\mathscr{R}_{1}$ for any $\psi$. Therefore $\operatorname{ind}_{\mathscr{R}_{1}}^{s} \rho_{1} \otimes \psi$ is irreducible for any $\psi$. Hence we conclude $\tau=$ $\operatorname{ind}_{\mathscr{R}_{1}}^{S} \rho_{1} \otimes \psi$ for suitable $\psi$. Moreover, since $\mathscr{S} / \mathscr{N}$ is abelian any character $\psi$ of $\mathscr{R}_{1} / \mathscr{N}$ extends to a character $\phi$ of $\mathscr{S} / \mathscr{N}$. Then, if $\phi$ is the extension of $\psi$ to $\mathscr{S} / \mathscr{N}$, clearly ind $\mathscr{\mathscr { R }}_{1} \rho_{1} \otimes \psi=\phi \otimes \operatorname{ind}_{\mathscr{R}_{1}}^{\mathscr{S}} \rho_{1}$. Thus all irreducible representations of $\mathscr{S}$ lying over $\sigma$ are induced from $\mathscr{R}_{1}$, and if $\tau_{1}$ and $\tau_{2}$ are two such, then $\tau_{2}=\phi \otimes \tau_{1}$ for a suitable character $\phi$ of $\mathscr{S} / \mathscr{N}$. We conclude from all this that if we can compute multiplicities in $L^{2}\left(\mathscr{R}_{1} / \mathscr{R}_{1} \cap \Gamma\right)$ of representations of $\mathscr{R}_{1}$ lying over $\sigma$ on $\mathscr{N}$, then by an application of Proposition 2.1 we can compute the multiplicities in $L^{2}(\mathscr{S} / \Gamma)$. Therefore, we now take $\mathscr{R}_{1}=\mathscr{S}$ and assume $\sigma \in \hat{\mathscr{N}}$ actually extends to a representation $\rho$ of $\mathscr{S}$.

REMarks. Since we have made two reductions to get from the general case to the case where $\sigma$ extends, it might appear that we would have to apply Proposition 2.1 twice to recover the general case from the special case. It is not hard to convince oneself that in fact only one application of Proposition 2.1 will be necessary.

Let $L^{2}(\mathscr{S} \mid \Gamma, \sigma)$ denote the largest $\mathscr{S}$-invariant subspace of $L^{2}(\mathscr{S} \mid \Gamma)$ on which $\mathscr{N}$ acts by a multiple of $\sigma$. Before computing multiplicities of particular representations, let us consider the general appearance of $L^{2}(\mathscr{S} \mid \Gamma, \sigma)$. This discussion will be analogous to that of [16], $\S 1$, but we can be more specific here. Let $L^{2}(\mathscr{N} \mid \Delta, \sigma)$ denote the largest $\mathscr{N}$-invariant subspace of $L^{2}(\mathscr{N} / \Delta)$ on which $\mathscr{N}$ acts by a multiple of $\sigma$. Since $\mathscr{N} / \Delta \cong \mathscr{N} \Gamma / \Gamma, \mathscr{N} \Gamma$ acts on $L^{2}(\mathscr{N} / \Delta)$, and by restriction $\mathscr{N} \Gamma$ acts on $L^{2}(\mathscr{N} / \Delta, \sigma)$. Since $\sigma$ extends to $\mathscr{S}$, a fortiori it extends to $\mathscr{N} \Gamma$. Thus we see that, under the action of $\mathscr{N} \Gamma, L^{2}(\mathscr{N} \mid \Delta, \sigma) \cong \sum_{i=1}^{l} a_{i} \sigma_{i}$, where the $\sigma_{i}, i=1, \cdots, l$, are various distinct extensions of $\sigma$ to $\mathscr{N} \Gamma$, and $\sum_{i=1}^{l} a_{i}$ is the multiplicity of $\sigma$ in $L^{2}(\mathscr{N} / \Delta, \sigma)$. Note too that we may write $\sigma_{i}=\sigma_{1} \otimes \psi_{i}$, where the $\psi_{i}$ are distinct linear characters of $\mathscr{N} \Gamma / \mathscr{N}$. We will look at the $\psi_{i}$ more closely later on in this section and in part 3.

It is clear (see also [16]) that the representation of $\mathscr{S}$ on

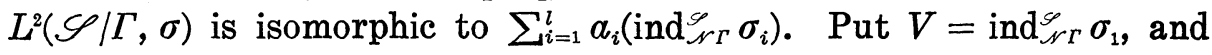
let $\rho_{1}$ be some irreducible component of $V$. Then if $\phi$ is any linear character of $\mathscr{S} \mid \mathscr{N} \Gamma$, clearly $\rho_{1} \otimes \phi$ also occurs in $V$. (One simply returns to the definition of induced representation and verifies that 
multiplication by $\phi$ preserves the appropriate transformation law.) On the other hand, the characters of $\mathscr{S} / \mathscr{N} \Gamma \operatorname{span} L^{2}(\mathscr{S} \mid \mathscr{N} \Gamma)$, and we may conclude from this that $V=\sum_{\phi} \rho_{1} \otimes \phi$, the sum being taken over all linear characters of $\mathscr{S} / \mathscr{N} \Gamma$. In other words, $V$ is multiplicity free and contains one copy of each possible extension of $\sigma_{1}$ from

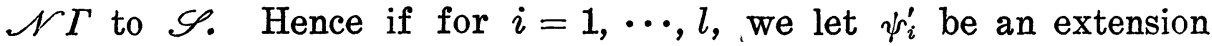
of $\psi_{i}$ from $\mathscr{N} \Gamma / \mathscr{N}$ to $\mathscr{S} / \mathscr{N}$, then we may write

$$
L^{2}(\mathscr{S} \mid \mathscr{N}, \sigma) \cong \sum_{i=1}^{l} \sum_{\phi} a_{i}\left(\rho_{1} \otimes \psi_{i}^{\prime} \otimes \phi\right),
$$

where $\phi$ again ranges over $(\mathscr{S} / \mathscr{N} \Gamma)^{\wedge}$. Computing multiplicities is thus seen to amount to determining a particular $\rho_{1}$ (or $\sigma_{1}$ ) and the $a_{i}$ 's and $\psi_{i}$ 's.

To accomplish this, we proceed in the general spirit of the discussion of the beginning of this part of the paper. We take a particular $\rho$ in $\hat{\mathscr{S}}$, lying above $\sigma$ in $\hat{\mathscr{F}}$, and we imagine that $\rho$ is induced from a representation $\tau$ of some subgroup $\mathscr{R}$ of $\mathscr{S}$. Then if $\tau_{1}$ is the representation of $\mathscr{N} \cdot \mathscr{R}$ induced from $\tau$ on $\mathscr{R}$, transitivity of induction shows $\tau_{1}$ induced to $\mathscr{S}$ yields $\rho$. Since $\rho$ restricted to $\mathscr{N}$ is already irreducible and equal to $\sigma$, we conclude that $\mathscr{R} \cdot \mathscr{N}=$ $\mathscr{S}, \tau_{1}=\rho$, and that if $\mathscr{C}=\mathscr{R} \cap \mathscr{N}$, then $\sigma=\operatorname{ind}_{\mathscr{N}}^{\mathscr{N}} \nu$, where $\nu$ is the restriction of $\tau$ to $\mathscr{M}$. Therefore $\nu$ must be irreducible, so $\tau$ is an extension of $\nu$ from $\mathscr{M}$ to $\mathscr{R}$. But now, notice that $\mathscr{R} \cdot \mathscr{N}=\mathscr{S}$ means that $\mathscr{R} / \mathscr{C}$ and $\mathscr{S} / \mathscr{N}$ are naturally isomorphic, and so therefore are their Pontryagin duals. Let $\tilde{\rho}$ be another representation of $\mathscr{S}$ lying over $\sigma$ on $\mathscr{N}$. Then $\tilde{\rho}=\rho \otimes \phi$ for some $\phi \in(\mathscr{S} \mid \mathscr{N})^{\wedge}$. Identifying $\phi$ to a character, also denoted $\phi$, of $\mathscr{R} / \mathscr{C}$ we see that if $\widetilde{\tau}=\tau \otimes \phi$, then $\tilde{\rho}$ on $\mathscr{S}$ is induced from $\tilde{\tau}$ on $\mathscr{R}$. That is, all representations of $\mathscr{S}$ lying over $\sigma$ on $\mathscr{N}$ are induced from $\mathscr{R}$ from extensions of $\nu$ from $\mathscr{C}$ to $\mathscr{R}$.

Now assume that $\mathscr{M}=\mathscr{R} \cap \mathscr{N}$ is connected, and that $\mathscr{R} \mid \mathscr{R} \cap \Gamma$ is compact. If this is so, then $\mathscr{M}|\mathscr{M} \cap \Gamma=\mathscr{M}| \mathscr{M} \cap \Delta$ will be compact too, as may be seen by the following argument, which is quite general. If $\mathscr{M} / \mathscr{M} \cap \Delta$ is not compact, then $\mathscr{M} \cdot(\mathscr{R} \cap \Gamma)$ is not closed in $\mathscr{R}$, and so $(\mathscr{R} \cap \Gamma) \cdot \mathscr{C} / \mathscr{M}$ is not closed in $\mathscr{R} / \mathscr{M}$. But $\mathscr{R} / \mathscr{M} \cong \mathscr{S} \mid \mathscr{N}$, and under this isomorphism $(\mathscr{R} \cap \Gamma) \cdot \mathscr{C} / \mathscr{M}$ is identified to a subset of $\Gamma \mathscr{N} / \mathscr{N}$. Since $\Gamma \mathscr{N} / \mathscr{N}$ is closed and discrete in $\mathscr{S} / \mathscr{N}$, any subset of it is closed. Hence $\mathscr{L} \cdot(\mathscr{R} \cap \Gamma)$ is indeed closed, and $\mathscr{C} \mid \mathscr{M} \cap \Delta$ is compact.

Given these circumstances, we want to establish a result like Proposition 2.1, or the result in the nilpotent case. This will take some work. To begin, we show how the existence of a good double coset $\mathscr{R} s \Gamma$ such that $\tau$ occurs in $L^{2}(\mathscr{R} \mid \mathscr{R} \cap \mathrm{Ad} s(\Gamma))$ gives rise to 
a subspace of the type of $\rho$ in $L^{2}(\mathscr{S} / \Gamma)$. Here we have put $\operatorname{Ad} s(\Gamma)=$ $s \Gamma s^{-1}$.

The basic idea is the same as in the case of a finite group: we construct an intertwining map. This was done explicitly by Richardson [34] in his discussion of the nilpotent group case. Also, L. Auslander [2] has explicitly pointed out the function of an intertwining map in the context of Proposition 1 of [16], and the present discussion benefits from his observation. As I mentioned above, my own discussion of multiplicities has so far, by a subterfuge, avoided intertwining questions entirely. However, they appear to be necessary here.

Consider a good double coset $\mathscr{R} s \Gamma$ such that $\tau$ occurs in $L^{2}(\mathscr{R} \mid \mathscr{R} \cap$ Ad $s(\Gamma))$. Replacing $\mathscr{R}$ by Ad $s^{-1}(\mathscr{R})$ and $\tau$ by the representation $\mathrm{Ad}^{*} s(\tau)$ of $\mathrm{Ad}^{-1}(\mathscr{R})$, which is defined by the formula Ad $^{*} s(\tau)\left(s^{-1} r s\right)=\tau(r)$ for $r \in \mathscr{R}$, we may suppose $s$ is the identity. Since $\tau$ occurs in $L^{2}(\mathscr{R} \mid \mathscr{R} \cap \Gamma), \rho$ occurs in $L^{2}(\mathscr{S} \mid \mathscr{R} \cap \Gamma)$. We try to embed $\rho$ in $L^{2}(\mathscr{S} \mid \Gamma)$ by averaging over $\Gamma / \mathscr{R} \cap \Gamma$. We can not do this directly because of problems of convergence. We proceed, slightly more cautiously, as follows. For a locally compact space $X$, let $C_{c}(X)$ denote the continuous functions of compact support on $X$. We define a map $\alpha: C_{c}(\mathscr{S} \mid \mathscr{R} \cap \Gamma) \rightarrow C_{c}(\mathscr{S} \mid \Gamma)$ by the formula

$$
\alpha(f)(s)=\sum_{\gamma \in I \mid \Gamma \cap \Omega_{\mathcal{R}}} f(s \gamma) .
$$

The series converges absolutely and uniformly to a function right invariant by $\Gamma$ (see [35]). It is clear that $\alpha$ commutes with the obvious actions of $G$ on the spaces $C_{c}(\mathscr{S} \mid \mathscr{R} \cap \Gamma)$ and $C_{c}(\mathscr{S} \mid \Gamma)$, and so we refer to $\alpha$ as a formal intertwining map or averaging map. We now attempt to obtain from $\alpha$ an actual intertwining map between $L^{2}(\mathscr{S} \mid \mathscr{R} \cap \Gamma)$ and $L^{2}(\mathscr{S} \mid \Gamma)$. We have $C_{c}(\mathscr{S} \mid \mathscr{R} \cap \Gamma) \subseteq$ $L^{2}(\mathscr{S} \mid \mathscr{R} \cap \Gamma)$ and $C_{o}(\mathscr{S} \mid \Gamma) \subseteq L^{2}(\mathscr{S} \mid \Gamma)$. Thus the graph of $\alpha$ sits inside $L^{2}(\mathscr{S} \mid \mathscr{R} \cap \Gamma) \times L^{2}(\mathscr{S} \mid \Gamma)$, and is an invariant subspace for $\mathscr{S}$ acting on this direct product. We consider the closure of the graph of $\alpha$. In general, this closure will have no properties at all. For example, if $\mathscr{S}=\boldsymbol{R}, \mathscr{R}=\{0\}$, and $\Gamma=Z$, then the closure of the graph of $\alpha$ is all of $L^{2}(\boldsymbol{R}) \times L^{2}(\boldsymbol{R} / \boldsymbol{Z})$. In our present context, however, we do get something useful. Recall that $\nu$ is the restriction of $\tau$ to $\mathscr{M}$. Let $L^{2}(\mathscr{R} / \mathscr{R} \cap \Gamma, \nu)$ be the subspace of $L^{2}(\mathscr{R} \mid \mathscr{R} \cap \Gamma)$ which transforms under $\mathscr{C}$ by $\nu$. Now of course the representation of $\mathscr{R}$ on $L^{2}(\mathscr{R} / \mathscr{R} \cap \Gamma)$ is just ind $\mathscr{R} \cap \Gamma 1$, and the representation of $\mathscr{S}$ on $L^{2}(\mathscr{S} \mid \mathscr{R} \cap \Gamma)$ is just ind $\mathscr{\mathscr { R }} \cap \Gamma$. Thus the representation of $\mathscr{S}$ which is induced from the representation of $\mathscr{R}$ acting on $L^{2}(\mathscr{R} \mid \mathscr{R} \cap \Gamma, \nu)$ is just a subrepresentation of $\mathscr{S}$ acting on $L^{2}(\mathscr{S} \mid \mathscr{R} \cap \Gamma)$. We denote the subspace of this represen- 
tation by $L^{2}(\mathscr{S} \mid \mathscr{R} \cap \Gamma, \nu)$. To give an alternate description, $L^{2}(\mathscr{S} \mid \mathscr{R} \cap \Gamma, \nu)$ consists of those functions $f \in L^{2}(\mathscr{S} \mid \mathscr{R} \cap \Gamma)$ such that for almost all $x \in \mathscr{S}$, the function $r \rightarrow f(x r)$ on $\mathscr{R}$ is in $L^{2}(\mathscr{R} \mid \mathscr{R} \cap \Gamma, \nu)$. Put

$$
C_{c}(\mathscr{S} \mid \mathscr{R} \cap \Gamma, \nu)=C_{c}(\mathscr{S} \mid \mathscr{R} \cap \Gamma) \cap L^{2}(\mathscr{S} \mid \mathscr{R} \cap \Gamma, \nu) .
$$

We will use similar notation for other homogeneous spaces in the course of the next few lemmas. For example, we have already defined $L^{2}(\mathscr{S} \mid \Gamma, \sigma)$ as the subspace of $L^{2}(\mathscr{S} \mid \Gamma)$ which transforms under $\mathscr{N}$ by $\sigma$. Similarly $C_{c}(\mathscr{S} \mid \Gamma, \sigma)=C_{c}(\mathscr{S} \mid \Gamma) \cap L^{2}(\mathscr{S} \mid \Gamma, \sigma)$. Clearly $\alpha\left(C_{c}(\mathscr{S} \mid \mathscr{R} \cap \Gamma, \nu)\right) \subseteq C_{e}(\mathscr{S} \mid \Gamma, \sigma)$. It is worth remarking explicitly on the simple general principal behind these definitions, for it will be very useful later, and helps clarify things. Namely, suppose $G_{1} \subseteq G_{2} \subseteq G_{3}$ are 3 locally compact groups, each closed in the next. Then if $V \cong L^{2}\left(G_{2} / G_{1}\right)$ is a left invariant subspace under the action of $G_{2}$, then the subspace of $L^{2}\left(G_{3} / G_{1}\right)$, consisting of functions $f$ such that for almost all $g_{3}$, the function $g_{2} \rightarrow f\left(g_{3} g_{2}\right)$ on $G_{2} / G_{1}$ is in $V$, is a well-defined subspace of $L^{2}\left(G_{3} / G_{1}\right)$, invariant under the action of $G_{3}$. Of course, this is simply part of the theorem on induction in stages.

Lemma 2.1. $C_{c}(\mathscr{S} \mid \mathscr{R} \cap \Gamma, \nu)$ is dense in $L^{2}(\mathscr{S} \mid \mathscr{R} \cap \Gamma, \nu)$. With proper normalization of measures, the restriction of the formal intertwining operator $\alpha$ to $C_{c}(\mathscr{S} \mid \mathscr{R} \cap \Gamma, \nu)$ extends to an isometric injection of $L^{2}(\mathscr{S} \mid \mathscr{R} \cap \Gamma, \nu)$ into $L^{2}(\mathscr{S} \mid \Gamma)$.

Proof. By the alternate description of $L^{2}(\mathscr{S} \mid \mathscr{R} \cap \Gamma, \nu)$ given just above, we see that those functions $f \in L^{2}(\mathscr{S} \mid \mathscr{R} \cap \Gamma, \nu)$ which are of compact support modulo $\mathscr{R} \cap \Gamma$ are dense in $L^{2}(\mathscr{S} \mid \mathscr{R} \cap \Gamma, \nu)$. For if $U$ is an open relatively compact set of $\mathscr{S}$, and

$$
f \in L^{2}(\mathscr{S} \mid \mathscr{R} \cap \Gamma, \nu),
$$

then the function which agrees with $f$ on $U \mathscr{R}$ and is zero outside $U \mathscr{R}$ is also in $L^{2}(\mathscr{S} \mid \mathscr{R} \cap \Gamma, \nu)$ since it transforms correctly under $\mathscr{R}$. Also it has compact support modulo $\mathscr{R} \cap \Gamma$, since $\mathscr{R} \cap \Gamma$ is compact. Now let $f \in L^{2}(\mathscr{S} \mid \mathscr{R} \cap \Gamma$, $\nu)$ have compact support modulo $\mathscr{R}$, and let $\beta \in C_{c}(\mathscr{S})$. Then $\beta * f$ is in

$$
L^{2}(\mathscr{S} \mid \mathscr{R} \cap \Gamma, \nu),
$$

still has compact support modulo $\mathscr{R}$, and is continuous. Now just let $\beta$ run through an approximate identity in $L^{1}(\mathscr{S})$, and $\beta * f$ will approach $f$. This proves the first statement of the lemma.

We first prove the second part of the lemma in the special case 
when $\Gamma=(\mathscr{R} \cap \Gamma) \cdot \Delta$ and $\mathscr{A}$ is normal in $\mathscr{N}$. By our assumptions, $\Gamma / \mathscr{R} \cap \Gamma \cong \Delta / \mathscr{A} \cap \Delta$, so we may choose our $\mathscr{R} \cap \Gamma$ coset representatives $\left\{\gamma_{i}\right\}_{i=1}^{\infty}$ to lie in $\Delta$. The $\gamma_{i}$ will then normalize $\mathscr{L} \cap \Delta$.

Now if $f \in C_{c}(\mathscr{S} \mid \mathscr{R} \cap \Gamma)$, and $h \in C_{c}(\mathscr{S} \mid \Gamma)$, then we have the formula (see [15], [35]):

$$
\int_{\mathscr{S} \mid \Gamma} \alpha(f) \bar{h} d \mu=\int_{\mathscr{S} \mid \mathscr{R} \cap \Gamma} f \bar{h} d x
$$

In this formula $d \mu$ is the standard probability measure on $\mathscr{S} / \Gamma$, and $d x$ is an appropriately normalized invariant measure on $\mathscr{S} \mid \mathscr{R} \cap \Gamma$. We regard $h$ alternately as a function of $\mathscr{S} \mid \Gamma$ or on $\mathscr{S} \mid \mathscr{R} \cap \Gamma$, and $\bar{h}$ is the complex conjugate of $h$. The formula expresses the relationship between inner products in $L^{2}(\mathscr{S} \mid \Gamma)$ and $L^{2}(\mathscr{S} \mid \mathscr{R} \cap \Gamma)$. We may ([15], [35]) rewrite the left hand side of (1):

$$
\int_{\mathscr{S} \mid \Gamma} \alpha(f) \bar{h} d \mu=\int_{S \mid \mathcal{S} \Gamma} d s\left(\int_{\mathscr{S} \mid \Lambda} \alpha(f)(s n) \bar{h}(s n) d \tilde{\mu}\right) .
$$

Here $d s$ and $d \tilde{\mu}$ are the standard invariant probability measures on $\mathscr{S} \mid \mathscr{N} \Gamma$ and $\mathscr{N} / \Delta$ respectively. Similarly, we may rewrite the right hand side of (1) as:

$$
\int_{\mathscr{S} \mid \operatorname{SR\cap } \Gamma} f \bar{h} d x=\int_{\mathscr{S} \mid \mathcal{N} \Gamma} d s\left(\int_{\mathscr{N} \mid \mathcal{M} \cap} f(s y) \bar{h}(s y) d y\right) .
$$

Here $d s$ is as in (2) and $d y$ is a suitable invariant measure on $\mathscr{N} /(\Delta \cap \mathscr{M})$.

Now if $f$ is a continuous function on $\mathscr{S}$, and $s \in \mathscr{S}$, define $f_{s}$ on $\mathscr{N}$ by $f_{s}(n)=f(s n)$. We have a map $\tilde{\alpha}: C_{c}(\mathscr{N} / \mathscr{L} \cap \Delta) \rightarrow C_{c}(\mathscr{N} / \Delta)$ defined by the formula $\tilde{\alpha}(h)(n)=\sum r_{i} h\left(n \gamma_{i}\right)$. Again, the sum converges absolutely and uniformly on compacta. Clearly for $f \in C_{c}(\mathscr{S} \mid \mathscr{R} \cap \Gamma)$, we have $f_{s} \in C_{c}(\mathscr{N} / \mathscr{C} \cap \Delta)$, and the formula $\alpha(f)_{s}(n)=\tilde{\alpha}\left(f_{s}\right)(n)$ holds. Using this notation, and using (2) and (3), we see that (1) becomes

$$
\int_{\mathscr{S} \mid \mathcal{N} T} d s\left(\int_{\mathscr{S} \mid \Delta} \tilde{\alpha}\left(f_{s}\right) \bar{h}_{s} d \tilde{\mu}\right)=\int_{\mathscr{S} \mid \mathcal{N} T} d s \int_{\mathscr{S} \mid \mathcal{M} \cap \Delta} f_{s} \bar{h}_{s} d y \text {. }
$$

Suppose that $h=\alpha\left(h^{\prime}\right)$ for some $h^{\prime} \in C_{c}(\mathscr{S} \mid \mathscr{R} \cap \Gamma)$. Then the righthand side of (4) becomes:

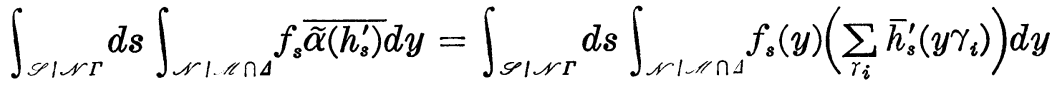

$$
\begin{aligned}
& =\int_{\mathscr{S} \mid \mathscr{N} \Gamma} d s\left(\sum_{\gamma_{i}} \int_{\mathscr{N | \mathscr { N } \cap \Delta}} f_{s}(y) \bar{h}_{s}^{\prime}\left(y \gamma_{i}\right) d y\right) \text {. }
\end{aligned}
$$

The rearrangement is legal because in fact $\Delta / \mathscr{L} \cap \Delta$ acts properly discontinuously on $\mathscr{N} / \mathscr{M} \cap \Delta$, and in fact, for a fixed $s$, only finitely 
many of the products $f_{s}(y) h_{s}^{\prime}\left(y \gamma_{i}\right)$ are nonzero. (We imagine that for the outer integral in (5), we have picked representatives for $s \in$ $\mathscr{S} \mid \mathscr{N} \Gamma$, and are integrating over a nice fundamental domain for $\mathscr{N} \Gamma$ in $\mathscr{S}_{\text {.) }}$ Thus we arrive at our final formula for $f$ and $h^{\prime} \epsilon$ $C_{c}(\mathscr{S} /(\mathscr{R} \cap \Gamma))$.

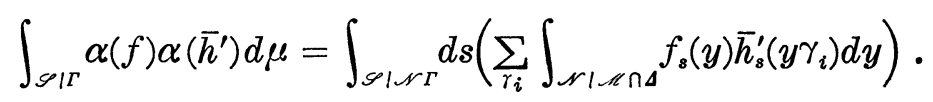

Take $f$ to be in $C_{c}(\mathscr{S} \mid \mathscr{R} \cap \Gamma, \nu)$. This means that for each $s \in \mathscr{S}$ and $n \in \mathscr{N}$, the function $m \rightarrow f_{s}(n m)$, for $m \in \mathscr{M}$ is in $C_{c}(\mathscr{L} \mid \mathscr{L} \cap \Delta, \nu)$, the functions in $C_{c}(\mathscr{H} \mid \mathscr{M} \cap \Delta)$ which belong to the $\nu$-isotypic component of $L^{2}(\mathscr{L} \mid \mathscr{C} \cap \Delta)$. Then, since $\gamma_{i}$ normalizes $\mathscr{M}$ and $\mathscr{C} \cap \Delta$, and $\operatorname{Ad}^{*} \gamma_{i}(\nu) \neq \nu$ since $\nu$ induces the irreducible representation $\sigma$ of $\mathscr{N}$, we see that $C_{c}\left(\mathscr{L} / \mathscr{L} \cap \Delta, \operatorname{Ad}^{*} \gamma_{i}(\nu)\right)$ is orthogonal to

$$
C_{0}(\mathscr{M} \mid \mathscr{M} \cap \Delta, \nu) \text {. }
$$

But if $f \in C_{c}(\mathscr{S} \mid \mathscr{R} \cap \Gamma, \nu)$, then $m \rightarrow f_{s}\left(n m \gamma_{i}\right)$ is in $C_{c}(\mathscr{M} \mid \mathscr{M} \cap$ $\left.\Delta, \operatorname{Ad}^{*} \gamma_{i}(\nu)\right)$. Therefore, by integrating first over the $\mathscr{C} / \mathscr{M} \cap \Delta$ fibres, then over $\mathscr{N} / \mathscr{M}$, we see that if $\gamma_{i}$ does not represent the identity,

$$
\int_{\mathscr{N} \mathfrak{M} \cap A} f_{s}(y) \bar{h}_{s}^{\prime}\left(y \gamma_{i}\right) d y=0
$$

for any $f, h^{\prime} \in C_{c}(\mathscr{S} \mid \mathscr{R} \cap \Gamma, \nu)$. Hence for such $f, h^{\prime}$, formula (6) reads

$$
\begin{aligned}
& \int_{\mathscr{S} \mid \Gamma} \alpha(f) \overline{\alpha\left(h^{\prime}\right)}=\int_{\mathscr{S} \mid \mathcal{N} \Gamma} d s \int_{\mathscr{N} \mid \not \cap A} f_{s}(y) \bar{h}_{s}^{\prime}(y) d y \\
& =\int_{\mathscr{S} \mid \mathscr{R} \cap \Gamma} f(x) \bar{h}^{\prime}(x) d x \text {. }
\end{aligned}
$$

Thus in the case we have been considering, namely $\mathscr{C}$ normal in $\mathscr{N}$, and $\Gamma=(\mathscr{R} \cap \Gamma) \cdot \Delta$, the lemma is established. We defer the proof in the general case in order to introduce another important property of our intertwining operators.

So far we have been considering a single $(\mathscr{R}, \Gamma)$ double coset, which we have for convenience normalized to be the identity coset. Suppose, however, that we have two good $(\mathscr{R}, \Gamma)$ double cosets $\mathscr{R} s_{1} \Gamma$ and $\mathscr{R} s_{2} \Gamma$ such that $\tau$ occurs in $L^{2}\left(\mathscr{R} \mid \mathscr{R} \cap\right.$ Ad $\left.s_{i}(\Gamma)\right)$ for $i=$ 1, 2. Put $\mathscr{R}_{i}=\mathrm{Ad} s_{i}^{-1} \mathscr{R}$. Then by choosing coset representatives for $\mathscr{R}_{i} \cap \Gamma$ in $\Gamma$, we can as above form averaging maps

$$
\alpha_{i}: C_{c}\left(\mathscr{S} \mid \mathscr{R}_{i} \cap \Gamma\right) \longrightarrow C_{c}(\mathscr{S} \mid \Gamma) \text {. }
$$


LEMMA 2.2. There are only finitely many good double cosets $\mathscr{R} s \Gamma$ such that $\tau$ occurs in $L^{2}(\mathscr{R} \mid \mathscr{R} \cap \mathrm{Ad} s(\Gamma))$. Suppose $\mathscr{R} s_{1} \Gamma$ and $\mathscr{R} s_{2} \Gamma$ are two distinct good double cosets for which $\tau$ does occur. Then the images of $\alpha_{i}: C_{c}\left(\mathscr{S} \mid \mathscr{R}_{i} \cap \Gamma, \mathrm{Ad}^{*} s_{i}(\nu)\right) \rightarrow C_{c}(\mathscr{S} \mid \Gamma)$ are orthogonal (with respect to the inner product $C_{c}(\mathscr{S} / \Gamma)$ inherits as a subspace of $\left.L^{2}(\mathscr{S} / \Gamma)\right)$.

Proof. Since $\mathscr{R} \cdot \mathscr{N}=\mathscr{S}$, any $(\mathscr{R}, \Gamma)$ double coset has a representative in $\mathscr{N}$. Let $\mathscr{R} n \Gamma, n \in \mathscr{N}$, be a $\operatorname{good}(\mathscr{R}, \Gamma)$ double coset. Then $\mathscr{R} / \mathscr{R} \cap \operatorname{Ad} n(\Gamma)$ is compact. This as we saw before implies $\mathscr{L} \mid \mathscr{C} \cap \operatorname{Ad} n(\Delta)$ is compact. Clearly $\tau$ can occur in $L^{2}(\mathscr{R} \mid \mathscr{R} \cap \operatorname{Ad} n(\Gamma))$ only if $\nu$ occurs in $L^{2}(\mathscr{C} / \mathscr{C} \cap \operatorname{Ad} n(\Delta))$. But we know from the nilpotent case that there are only finitely many good $(\mathscr{M}, \Delta)$ double cosets such that $\nu$ occurs in $L^{2}(\mathscr{C} / \mathscr{M} \cap \operatorname{Ad} n(\Delta))$ with positive multiplicity. This proves the first statement of the lemma.

To prove the second statement, we again begin by taking $\mathscr{C}$ to be normal in $\mathscr{N}$. The argument follows the same lines as in Lemma 2.1. We run through it somewhat faster. 'Let $\left\{\gamma_{j}\right\}_{j=1}^{\infty}$ be a set of coset representatives for $\mathscr{C} \cap \Delta$ in $\Delta$. Then $\left\{\gamma_{j}\right\}$ will also be a set of coset representatives for $\mathscr{R}_{i} \cap \Gamma$ in $\left(\mathscr{R}_{i} \cap \Gamma\right) \cdot \Delta=\Gamma_{i}$. As $\Gamma_{2}$ is of finite index $l$ in $\Gamma$, we may choose a finite set $\left\{\eta_{k}\right\}_{k=1}^{l}$ of coset representatives for $\Gamma_{2}$ in $\Gamma$. Then $\left\{\eta_{k} \gamma_{j}\right\}_{j=1, k=1}^{\infty}$ are a set of coset representatives for $\mathscr{R}_{2} \cap \Gamma$ in $\Gamma$. Of course, precisely the same set-up applies to $\Gamma_{1}$, but we do not need to make this explicit in our arguments.

Take $f_{i} \in C_{c}\left(\mathscr{S} \mid \mathscr{R}_{i} \cap \Gamma\right)$. For $f_{2}$, we have the formula $\alpha_{2}\left(f_{2}\right)(x)=$ $\sum_{j, k} f\left(x \eta_{k} \gamma_{j}\right)$. We compute

$$
\begin{aligned}
& \int_{\mathscr{S} \mid \Gamma} \alpha_{1}\left(f_{1}\right) \alpha_{2}\left(\bar{f}_{2}\right) d \mu=\int_{\mathscr{S} \mid \mathscr{R}_{1} \cap \Gamma} f_{1} \alpha_{2}\left(\bar{f}_{2}\right) d x_{1} \\
& =\int_{\mathscr{S} \mid \mathscr{N} \Gamma_{1}} d s_{1}\left(\int_{\mathscr{N | N \cap A}} f_{1}(s y) \alpha_{2}\left(\bar{f}_{2}\right)(s y) d y\right)
\end{aligned}
$$

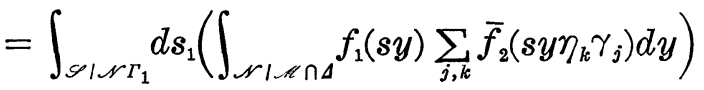

$$
\begin{aligned}
& =\int_{\mathscr{S} \mid \mathscr{N} \Gamma_{1}} d s_{1}\left(\sum_{j, k} \int_{\mathscr{N} \mid \mathbb{M} \Lambda} f_{1}(s y) \bar{f}_{2}\left(s y \eta_{k} \gamma_{j}\right) d y\right) \text {. }
\end{aligned}
$$

In the above $d \mu, d x_{1}, d s_{1}$, and $d y$ all denote appropriately normalized invariant measures on the relevant coset spaces. Consider a typical term in the inner expression of the last member in (8). We see

$$
\begin{aligned}
& \int_{\mathscr{\sim} \mid \mathbb{N} \cap \Delta} f_{1}(s y) \bar{f}_{2}\left(s y \eta_{k} \gamma_{j}\right) d y
\end{aligned}
$$

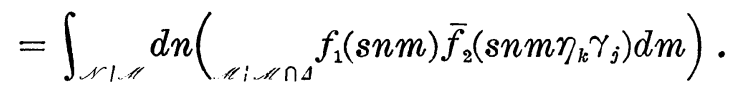


Here again $d n$ and $d m$ are the obvious invariant measures. Now the function $m \rightarrow f_{1}(s n m)$ belongs to $C_{0}\left(\mathscr{M} \mid \mathscr{M} \cap \Delta, \mathrm{Ad}^{*} n_{1}(\nu)\right)$, while $m \rightarrow f_{2}\left(s n m \eta_{k} \gamma_{j}\right)$ is in $C_{o}\left(\mathscr{L} \mid \mathscr{M} \cap \Delta, \mathrm{Ad}^{*} \eta_{k} \gamma_{j} n_{2}(\nu)\right)$. The fact that $\nu$ extends to $\tau$ on $\mathscr{R}$, but induces $\sigma$ on $\mathscr{N}$, shows that the isotropy group of $\nu$ under $\operatorname{Ad}^{*} \mathscr{S}$ acting on $\hat{\mathscr{C}}$ is precisely $\mathscr{R}$. Thus the $(\mathscr{R}, \Gamma)$ double cosets correspond bijectively to $\mathrm{Ad}^{*} \Gamma$ orbits in $\operatorname{Ad}^{*} \mathscr{S}(\nu)$. Since $n_{1}$ and $n_{2}$ represent different $(\mathscr{R}, \Gamma)$ double cosets, $\operatorname{Ad}^{*} n_{1}(\nu) \neq \operatorname{Ad}^{*} \eta_{k} \gamma_{j} n_{2}(\nu)$ for any choice of $\eta_{k}$ or $\gamma_{j}$. Hence the inner integral in the right hand side of (9) is always zero, and plugging this fact in (8) yields the lemma in this case of $\mathscr{C}$ normal in $\mathscr{N}$.

Using the above, we now establish Lemma 2.1 completely. Then we will return to Lemma 2.2. For the moment we still take $\mathscr{C}$ normal in $\mathscr{N}$, but allow the possibility that $(\mathscr{R} \cap \Gamma) \cdot \Delta \neq \Gamma$. Again let $\left\{\gamma_{j}\right\}$ be coset representatives for $\mathscr{C} \cap \Delta$ in $\Delta$, and let $\left\{\eta_{k}\right\}_{k=1}^{l}$ here denote a set of coset representatives for $\Gamma^{0}=(\mathscr{R} \cap \Gamma) \cdot \Delta$ in $\Gamma$. Then we may express the averaging map $\alpha: C_{c}(\mathscr{S} \mid \mathscr{R} \cap \Gamma) \rightarrow C_{c}(\mathscr{S} \mid \Gamma)$ as a composite of two maps $\alpha^{0}: C_{c}(\mathscr{S} \mid \mathscr{R} \cap \Gamma) \rightarrow C_{c}\left(\mathscr{S} \mid \Gamma^{0}\right)$ and $\beta: C_{c}\left(\mathscr{S} \mid \Gamma^{0}\right) \rightarrow$ $C_{c}(\mathscr{S} \mid \Gamma)$. The maps $\alpha^{0}$ and $\beta$ are expressed by the expected formulas: $\alpha^{0}(f)(x)=\sum_{j} f\left(x \gamma_{j}\right)$ and $\beta(f)(x)=\sum_{k=1}^{l} f\left(x \eta_{k}\right)$ for $f$ in the domain of $\alpha^{0}$ or $\beta$. Since $\Gamma^{0}$ is normal in $\Gamma, \mathscr{R} \Gamma$ decomposes into $l$ distinct $\left(\mathscr{R}, \Gamma^{0}\right)$ cosets, namely $\mathscr{R} \eta_{k} \Gamma^{0}, k=1, \cdots, l$. If $\mathscr{R}_{k}=\operatorname{Ad} \eta_{k}^{-1}(\mathscr{R})$, then $\mathscr{R}_{k} \cap \Gamma^{0}=\operatorname{Ad} \eta_{k}^{-1}\left(\mathscr{R} \cap \Gamma^{0}\right)$, and we may define $\alpha_{k}^{0}(f) \in C_{c}\left(\mathscr{S} \mid \Gamma^{0}\right)$ for $f \in C_{c}\left(\mathscr{S} \mid \mathscr{R}_{k} \cap \Gamma\right)$ by the same formula as for $f \in C_{c}(\mathscr{S} \mid \mathscr{R} \cap \Gamma)$. (We note that $\left(\mathscr{R}_{k} \cap \Gamma\right) \cdot \Delta=\Gamma^{0}$ independently of $k$, again by the normality of $\Gamma^{0}$ in $\Gamma$.)

Now if $f \in C_{c}(\mathscr{S} \mid \mathscr{R} \cap \Gamma)$, then if $f_{k}(x)=f\left(x \eta_{k}\right), f_{k} \in C_{o}\left(\mathscr{S} \mid \mathscr{R}_{k} \cap \Gamma\right)$, and moreover $\alpha^{0}\left(f_{k}\right)(x)=\alpha^{0}(f)\left(x \eta_{k}\right)$. Therefore $\alpha(f)=\beta\left(\alpha^{0}(f)\right)=$ $\alpha^{0}(\beta(f))=\sum_{k} \alpha^{0}\left(f_{k}\right)$. So now if $f, h \in C_{c}(\mathscr{S} \mid \mathscr{R} \cap \Gamma)$, then

$$
\begin{gathered}
\int_{\mathscr{S} \mid \Gamma} \alpha(f) \alpha(\bar{h}) d \mu=\int_{\mathscr{S} \mid \Gamma^{0}} \alpha^{0}(f) \alpha(\bar{h}) d \mu^{0} \\
=\sum_{k} \int_{\mathscr{S} \mid \Gamma^{0}} \alpha^{0}(f) \alpha^{0}\left(h_{k}\right) d \mu^{0} .
\end{gathered}
$$

Take now $f, h \in C_{c}(\mathscr{S} \mid \mathscr{R} \cap \Gamma, \nu)$. Then $h_{k}$ belongs to $C_{c}\left(\mathscr{S} \mid \mathscr{R}_{k} \cap\right.$ $\left.\Gamma, \mathrm{Ad}^{*} \eta_{k}(\nu)\right)$. The case of Lemma 2.2 which we have established now guarantees that in the sum in the last member of (10), only the $k$ for which $\eta_{k} \in \Gamma^{0}$ gives a nonzero contribution, and Lemma 2.1 is now completely proven when $\mathscr{M}$ is normal in $\mathscr{N}$.

When $\mathscr{C}$ is not normal in $\mathscr{N}$, we can pull ourselves up in stages. If $\mathscr{C}^{0}=\mathscr{C}$ and $\mathscr{M}^{i+1}$ is the normalizer of $\mathscr{M}^{i}$ in $\mathscr{N}$, then the $\mathscr{M}^{i}$ have the following properties. $\mathscr{M}^{l}=\mathscr{N}$ for some finite $l$, and $\mathscr{M}^{i}$ is normalized by $\mathscr{R}$. The quotients $\mathscr{C}^{i} / \mathscr{C}^{i} \cap \Delta$ are compact. If $\mathscr{R}^{i}=\mathscr{R} \cdot \mathscr{C}^{i}$ then $\mathscr{R}^{i}$ is a closed subgroup of $\mathscr{R}^{i+1}$, and $\mathscr{R}^{i} / \mathscr{R}^{i} \cap \Gamma$ is compact. Put $\nu^{i}=\operatorname{ind}_{\mathscr{M}}^{i} \nu$. Then $\nu^{i}$ is 
irreducible and induces $\sigma$ on $\mathscr{N}$ and $\nu^{i+1}$ on $\mathscr{C}^{i+1}$. For each $i$, we can average over cosets of $\mathscr{R}^{i} \cap \Gamma$ in $\mathscr{R}^{i+1} \cap \Gamma$ and this will produce maps $\tilde{\alpha}^{i}: C_{e}\left(\mathscr{R}^{i+1} / \mathscr{R}^{i} \cap \Gamma\right) \rightarrow C_{e}\left(\mathscr{R}^{i+1} / \mathscr{R}^{i+1} \cap \Gamma\right)$ and $\alpha^{i}: C_{e}\left(\mathscr{S} \mid \mathscr{R}^{i} \cap \Gamma\right) \rightarrow C_{e}\left(\mathscr{S} \mid \mathscr{R}^{i+1} \cap \Gamma\right)$. Of course the maps $\alpha^{i}$ and $\tilde{\alpha}^{i}$ are closely related. For a given $s \in \mathscr{S}, f \in C_{c}\left(\mathscr{S} \mid \mathscr{R}^{i} \cap \Gamma\right)$, define $f_{s}(r)=f(s r)$ for $r \in \mathscr{R}^{i+1}$. Then $f_{s} \in C_{c}\left(\mathscr{R}^{i+1} / \mathscr{R}^{i} \cap \Gamma\right)$. Similarly, if $h \in C_{c}\left(\mathscr{S} / \mathscr{R}^{i+1} \cap \Gamma\right), h_{s}(r)=h(s r)$ is in $C_{c}\left(\mathscr{R}^{i+1} / \mathscr{R}^{i+1} \cap \Gamma\right)$, and we have, for proper normalization of the various measures, the integral formula

$$
\begin{aligned}
\int_{\mathscr{S} \mid \mathscr{Z}^{i+1} \cap \Gamma} \alpha^{i}(f)(y) h(y) d y & =\int_{\mathscr{S} \mid \mathscr{R}^{i+1}} d x\left(\int_{\mathscr{R}^{i+1 / \mathscr{R}^{i+1} \cap \Gamma}} \tilde{\alpha}^{i}\left(f_{x}\right)(r) h_{x}(r) d r\right) .
\end{aligned}
$$

We know that $\tilde{\alpha}^{i}$ restricted to $C_{c}\left(\mathscr{R}^{i+1} / \mathscr{R}^{i} \cap \Gamma, \nu^{i}\right)$ is an isometric injection of this space (with the pre-Hilbert norm it has as a subspace of $\left.L^{2}\left(\mathscr{R}^{i+1} / \mathscr{R}^{i} \cap \Gamma\right)\right)$ into $C_{c}\left(\mathscr{R}^{i+1} / \mathscr{R}^{i+1} \cap \Gamma, \nu^{i+1}\right)$. We conclude from (11) that $\alpha^{i}: C^{o}\left(\mathscr{S} \mid \mathscr{R}^{i} \cap \Gamma, \nu^{i}\right) \rightarrow C_{c}\left(\mathscr{S} \mid \mathscr{R}^{i+1} \cap \Gamma, \nu^{i+1}\right)$ is also an isometric injection. Now composing the $\alpha^{i}$ 's gives Lemma 2.1 completely.

Now we finish the proof of Lemma 2.2. The procedure is much the same as the above, but a slightly different formulation is convenient.

Let $\mathscr{R} n_{i} \Gamma, i=1,2, n_{i} \in \mathscr{N}$ be two good double cosets such that $\tau$ occurs in $L^{2}\left(\mathscr{R} \mid \mathscr{R} \cap \operatorname{Ad} n_{i}(\Gamma)\right)$. Let $\mathscr{R}_{i}=\operatorname{Ad} n_{i}^{-1}(\mathscr{R})$. With the $\mathscr{K}^{i}$ as defined above, if $\mathscr{C}^{l}=\mathscr{N}$, then $\mathscr{L}^{l-1}$ is normal in $\mathscr{N}$ and $\mathscr{R}_{i} \cap \mathscr{N} \subseteq \mathscr{C}^{l-1}$. Put $\mathscr{R}^{\prime}=\mathscr{R} \cdot \mathscr{C}^{l-1}$ and $\mathscr{R}_{i}^{\prime}=\mathscr{R}_{i} \cdot \mathscr{M}^{l-1}$. By picking a set of coset representatives for $\mathscr{R}_{i} \cap \Gamma$ in $\mathscr{R}_{i}^{\prime} \cap \Gamma$, we get averaging maps $\alpha_{i}^{\prime}: C_{c}\left(\mathscr{S} \mid \mathscr{R}_{i} \cap \Gamma\right) \rightarrow C_{c}\left(\mathscr{S} \mid \mathscr{R}_{i}^{\prime} \cap \Gamma\right)$ and

$$
\tilde{\alpha}_{i}^{\prime}: C_{c}\left(\mathscr{R}_{i}^{\prime} \mid \mathscr{R}_{i} \cap \Gamma\right) \longrightarrow C_{c}\left(\mathscr{R}_{i}^{\prime} \mid \mathscr{R}_{i}^{\prime} \cap \Gamma\right) \text {. }
$$

Similarly, by picking coset representatives for $\mathscr{R}_{i}^{\prime} \cap \Gamma$ in $\Gamma$, we get averaging maps $\beta_{i}: C_{c}\left(\mathscr{S} \mid \mathscr{R}_{i}^{\prime} \cap \Gamma\right) \rightarrow C_{c}(\mathscr{S} \mid \Gamma)$. It is clear that the composites $\beta_{i}{ }^{\circ} \alpha_{i}^{\prime}=\alpha_{i}$ are just the averaging maps from $C_{c}\left(\mathscr{S} \mid \mathscr{R}_{i} \cap \Gamma\right)$ to $C_{o}(\mathscr{S} \mid \Gamma)$ with which we are concerned.

There are two possibilities: either $\mathscr{R}^{\prime} n_{1} \Gamma$ and $\mathscr{R}^{\prime} n_{2} \Gamma$ are the same, or they are not. Suppose first that they are not. Then $\tilde{\alpha}_{i}^{\prime}\left(C_{c}\left(\mathscr{R}_{i}^{\prime} \mid \mathscr{R}_{i} \cap \Gamma, \operatorname{Ad}^{*} n_{i}(\nu)\right)\right) \subseteq C_{c}\left(\mathscr{R}_{i}^{\prime} \mid \mathscr{R}_{i}^{\prime} \cap \Gamma, \operatorname{Ad}^{*} n_{i}\left(\nu^{l-1}\right)\right)$, with $\nu^{l-1}$ as above. Thus

$$
\alpha_{i}\left(C_{c}\left(\mathscr{S} \mid \mathscr{R}_{i} \cap \Gamma, \mathrm{Ad}^{*} n_{i}(\nu)\right)\right) \subseteq C_{c}\left(\mathscr{S} \mid \mathscr{R}_{i}^{\prime} \cap \Gamma, \operatorname{Ad}^{*} n_{i}\left(\nu^{l-1}\right)\right) .
$$

But since $\mathscr{C}^{l-1}$ is normal in $\mathscr{N}$, the images under the $\beta_{i}$ of the spaces $C_{c}\left(\mathscr{S} \mid \mathscr{R}_{i}^{\prime} \cap \Gamma, \mathrm{Ad}^{*} n_{i}\left(\nu^{l-1}\right)\right)$ are orthogonal by the special case of Lemma 2.2 which we have already established. 
So consider the other possibility, that $\mathscr{R}^{\prime} n_{1} \Gamma=\mathscr{R}^{\prime} n_{2} \Gamma$. Then if we choose $n_{1}$ and $n_{2}$ correctly, $n_{2}=m n_{1}$ with $m \in \mathscr{M}^{l-1}$. Taking this choice, we see that $\mathscr{R}_{1}^{\prime}=\mathscr{R}_{2}^{\prime}$ and that $\mathscr{R}_{1}$ and $\mathscr{R}_{2}$ are conjugate in $\mathscr{R}_{1}^{\prime}$. This being so, we may make the inductive assumption that the images of $\widetilde{\alpha}_{1}^{\prime}$ and $\tilde{\alpha}_{2}^{\prime}$ in $C_{c}\left(\mathscr{R}_{1}^{\prime} / \mathscr{R}_{1}^{\prime} \cap \Gamma, \mathrm{Ad}^{*} n_{1}\left(\nu^{l-1}\right)\right)$ are orthogonal. Then integrating fibrewise, we see that the images of $\alpha_{i}^{\prime}$ in $C_{c}\left(\mathscr{S} \mid \mathscr{R}_{1}^{\prime} \cap\right.$ $\left.\Gamma, \operatorname{Ad}^{*} n_{1}\left(\nu^{-1}\right)\right)$ are orthogonal. Now we apply Lemma 2.1, which says $\beta_{1}$ is an isometric injection of $C_{c}\left(\mathscr{S} \mid \mathscr{R}_{1}^{\prime} \cap \Gamma, \operatorname{Ad}^{*} n_{1}\left(\nu^{l-1}\right)\right)$ into $C_{c}(\mathscr{S} \mid \Gamma)$. Since $\alpha_{i}=\beta_{1}^{\circ} \alpha_{i}^{\prime}$, the images of the $\alpha_{i}$ in $C_{c}(\mathscr{S} / \Gamma)$ are orthogonal, and Lemma 2.2 is established.

Having seen how to construct subspaces of $L^{2}(\mathscr{S} \mid \Gamma, \sigma)$ using good $(\mathscr{R}, \Gamma)$ double cosets, we want to show this is not an empty construction. Indeed, we will show that the spaces we construct in this way exhaust $L^{2}(\mathscr{S} / \Gamma, \sigma)$. From this fact to the multiplicity formula we are after is but a short step. We base the result on Richardson's parallel result ([34]) for the nilpotent case. The main point in applying [34] is the next lemma, guaranteeing the existence of enough good $(\mathscr{R}, \Gamma)$ double cosets. It is this argument, which uses strongly certain facts about algebraic groups, that seems the hardest to transfer to less structured (e.g., more general) context. I do not even know how to prove it for a solvable group $\mathscr{S}$ if $\mathrm{Ad}^{*} \mathscr{S}$ does not leave $\sigma$ fixed. That is the reason for preliminary reduction to this case.

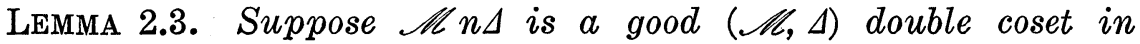
$\mathscr{N}$ such that $\nu$ occurs in $L^{2}(\mathscr{M} \mid \mathscr{M} \cap \operatorname{Ad} n(\Delta))$. Then $\mathscr{R} n \Gamma$ is a $\operatorname{good}(\mathscr{R}, \Gamma)$ double coset.

Proof. Let $\mathscr{\mathscr { L }}(\mathscr{N})$ denote the center of $\mathscr{N}$, and write $\overline{\mathscr{S}}=$ $\mathscr{S} / \mathscr{X}(\mathscr{N})$. If $X \cong \mathscr{S}$ is any subset, let $\bar{X} \cong \mathscr{\mathscr { S }}$ denote the image of $X$ under the natural projection of $\mathscr{S}$ onts $\overline{\mathscr{S}}$. Since $\sigma$ is induced from $\nu$ on $\mathscr{K}, \mathscr{C}$ must contain $\mathscr{Z}(\mathscr{N})$. Thus $\mathscr{R} n \Gamma$ is the full inverse image in $\mathscr{S}$ of $\overline{\mathscr{R}} \bar{\Gamma} \cong \overline{\mathscr{S}}$. Since $\mathscr{R} n \Gamma$ is good means it is closed, and likewise for $\overline{\mathscr{R}} \bar{n} \bar{\Gamma}$, we see that if we can show $\overline{\mathscr{R}} \bar{n} \bar{\Gamma}$ is good, we will have proved the lemma.

As is well-known ([24], [26]), the subgroup $\Delta$ of $\mathscr{N}$ endows $\mathscr{N}$ with the structure of unipotent algebraic group over $Q$ such that $\Delta$ is an arithmetic subgroup of $\mathscr{N}$. This means that if $N$ is the Lie algebra of $\mathscr{N}$, then $N$ has the structure of vector space defined over $Q$. Let log $\Delta \cong N$ be the inverse image of $\Delta$ under the exponential map from $N$ to $\mathscr{N}$. Let $L$ be the additive subgroup of $N$ generated by $\log \Delta$. Then $L$ is a lattice in $N$. That is $L$ spans $N$ (over $\boldsymbol{R}$ ) and is discrete in $N$. The set of $Q$-rational points of $N$ 
are those points $x$ such that $t x \in L$ for some integer $t$. A basis over $\boldsymbol{Z}$ for $L$ is a basis over $Q$ for the $Q$-rational points of $N$ and is a basis over $\boldsymbol{R}$ for $N$.

Consider the adjoint action of $\mathscr{S}$ on $N$. The identity component of the kernel of Ad: $\mathscr{S} \rightarrow \operatorname{Hom}(N, N)$ is precisely $\mathscr{Z}(\mathscr{N})$, by the definition of nilradical. Thus the identity component of $\overline{\mathscr{S}}$ acts faithfully on $N$.

To show $\overline{\mathscr{R}} \bar{n} \bar{\Gamma}$ is good, it will be enough to show that we can choose the coset representative $n$ so that $\operatorname{Ad} \bar{n}(\bar{\Gamma}) \cap \bar{\Gamma}$ is of finite index in $\bar{\Gamma}$. For then $\overline{\mathscr{R}} \cap \bar{\Gamma} \cap \operatorname{Ad} \bar{n}(\bar{\Gamma})$ has finite index in $\overline{\mathscr{R}} \cap \bar{\Gamma}$, so $\overline{\mathscr{R}} \mid \overline{\mathscr{R}} \cap \operatorname{Ad} \bar{n}(\bar{\Gamma})$ will be compact, another criterion of the goodness of $\overline{\mathscr{R}} \bar{n} \bar{\Gamma}$.

Let $\bar{\Gamma}_{1}$ be the subgroup of $\overline{\mathscr{S}}$ consisting of elements $\gamma$ such that Ad $\gamma(L)=L$. By the construction of $L$, we have $\bar{\Gamma} \cong \bar{\Gamma}_{1}$. On the other hand, the automorphisms of $L$ form a discrete group in $\operatorname{Hom}(N, N)$. Since the connected component of $\overline{\mathscr{S}}$ acts faithfully on $N$, it follows that $\bar{\Gamma}_{1}$ is discrete in $\overline{\mathscr{S}}_{\text {. }}$ Hence $\bar{\Gamma}$ is of finite index in $\bar{\Gamma}_{1}$. A simple argument then shows that if we can choose the double coset representative $n$ so that $\operatorname{Ad} \bar{n}\left(\bar{\Gamma}_{1}\right) \cap \bar{\Gamma}_{1}$ has finite index in $\bar{\Gamma}_{1}$, the same is true for $\bar{\Gamma}$. But $\operatorname{Ad} \bar{n}\left(\bar{\Gamma}_{1}\right) \cap \bar{\Gamma}_{1}$ will have finite index in $\bar{\Gamma}_{1}$ if $\operatorname{Ad} n(L) \cap L$ has finite index in $L$; for then $\operatorname{Ad} \bar{n}\left(\bar{\Gamma}_{1}\right) \cap$ $\bar{\Gamma}_{1}$ will contain some congruence subgroup of $\bar{\Gamma}_{1}$. Next, we see Ad $n(L) \cap L$ will be of finite index in $L$ if $n \in \mathscr{N}$ is a $Q$-rational point. So we are reduced to showing we can choose $n$ to be $Q$ rational.

Since $\nu$ induces $\sigma$, it follows that the set of pairs $(\operatorname{Ad} n(\mathscr{C})$, $\mathrm{Ad}^{*} n^{-1}(\nu)$ ) is naturally isomorphic to the space $\mathscr{N} / \mathscr{M}$. (See [16].) Since $\mathscr{M} / \mathscr{M} \cap \Delta$ is compact, $\mathscr{C}$ is defined over $Q$, and $\mathscr{N} / \mathscr{M}$ has the structure of variety over $Q$. This statement that $\mathscr{L} n \Delta$ is good and $\nu$ occurs in $L^{2}(\mathscr{C} / \mathscr{C} \cap \mathrm{Ad} n(\Delta))$ implies that (Ad $n(\mathscr{C}), \mathrm{Ad}^{*} n^{-1}(\nu)$ ) is a $Q$-rational point of $\mathscr{N} / \mathscr{M}$. It is however, well-known (see [14], and [24] for the role this plays in harmonic analysis on the adeles of a unipotent group) that the $Q$-rational points of $\mathscr{N}$ act transitively on the $Q$-rational points of $\mathscr{N} / \mathscr{M}$. Hence we may choose $n$ rational, and the lemma follows.

Now enumerate the good $(\mathscr{R}, \Gamma)$ double cosets $\left\{\mathscr{R} n_{i} \Gamma\right\}_{i=1}^{k}$ such that $\nu$ occurs with positive multiplicity in $L^{2}\left(\mathscr{C} \mid \mathscr{M} \cap \operatorname{Ad} n_{i}(\Delta)\right)$. We saw in Lemma 2.2 that these cosets were finite in number. Put $\mathscr{R}_{i}=\operatorname{Ad} n_{i}^{-1}(\mathscr{R})$ and $\mathscr{M}_{i}=\mathscr{R}_{i} \cap \mathscr{N}$, and let $\nu_{i}=\operatorname{Ad}^{*} n_{i}(\nu)$. We have shown that the averaging maps $\alpha_{i}: C_{c}\left(\mathscr{S} \mid \mathscr{R}_{i} \cap \Gamma, \nu_{i}\right) \rightarrow C_{c}(\mathscr{S} \mid \Gamma, \sigma)$ extend to isometric injections $\beta_{i}: L^{2}\left(\mathscr{S} \mid \mathscr{R}_{i} \cap \Gamma, \nu_{i}\right) \rightarrow L^{2}(\mathscr{S} \mid \Gamma, \sigma)$, and moreover the images of the $\beta_{i}$ are orthogonal spaces. 
LEMma 2.4. The images of the $\beta$ span $L^{2}(\mathscr{S} \mid \Gamma, \sigma)$. That is, we have a direct sum decomposition

$$
L^{2}(\mathscr{S} \mid \Gamma, \sigma)=\bigoplus_{i=1}^{k} \beta_{i}\left(L^{2}\left(\mathscr{S} \mid \mathscr{R}_{i} \cap \Gamma, \nu_{i}\right)\right)
$$

Proof. For a given $i$, put $\Gamma_{i}=\left(\mathscr{R}_{i} \cap \Gamma\right) \cdot \Delta$. Then $\Gamma_{i}$ has finite index $l(i)$ in $\Gamma$. Let $\left\{\eta_{i j}\right\}_{j=1}^{l(i)}$ be a set of coset representatives for $\Gamma_{i}$. We suppose $\eta_{i l}$ is the identity. By choosing coset representatives for $\mathscr{M}_{i} \cap \Delta$ in $\Delta$, we get averaging maps $\alpha_{i}^{0}: C_{c}\left(\mathscr{S} \mid \mathscr{R}_{i} \cap \Gamma\right) \rightarrow C_{c}\left(\mathscr{S} \mid \Gamma_{i}\right)$, and $\tilde{\alpha}_{i}^{0}: C_{c}\left(\mathscr{N} / \mathscr{C}_{i} \cap \Delta\right) \rightarrow C_{c}(\mathscr{N} / \Delta)$. The relations between these maps and the $\alpha_{i}$ above are as follows. If $f \in C_{c}\left(\mathscr{S} \mid \mathscr{R}_{i} \cap \Gamma\right)$, then $\alpha_{i}(f)(x)=$ $\sum_{j=1}^{l(i)} \alpha_{1}^{0}(f)\left(x \eta_{i j}\right)$. For $s \in \mathscr{S}$, if the function $f_{s}: n \rightarrow f(s n)$ is in $C_{c}\left(\mathscr{N} \mid \mathscr{A}_{i} \cap \Delta\right)$, and $\tilde{\alpha}_{i}^{0}\left(f_{s}\right)=\alpha_{i}^{0}(f)_{s}$.

We recall that $L^{2}(\mathscr{S} \mid \Gamma, \sigma)$ is the subspace of functions which transform by $\sigma$ under $\mathscr{N}$. Since $\sigma$ is invariant by $\mathrm{Ad}^{*} \mathscr{S}$, we may also describe $L^{2}(\mathscr{S} \mid \Gamma, \sigma)$ as the space consisting of $h \in L^{2}(\mathscr{S} \mid \Gamma)$, such that for almost all $s$, the function $n \rightarrow h(s n)$ on $\mathscr{N} / \Gamma$ is in $L^{2}(\mathscr{N} / \Gamma, \sigma)$. We want to divide $L^{2}(\mathscr{S} \mid \Gamma, \sigma)$ into certain invariant subspaces. To this end, let us consider those good $(\mathscr{C}, \Delta)$ doublecosets $\mathscr{C} n \Delta$ such that $\nu$ occurs in $L^{2}(\mathscr{M} \mid \mathscr{M} \cap \operatorname{Ad} n(\Delta))$. By Lemma 2.3, each such $\mathscr{L} x \Delta$ is contained in some $\mathscr{R} n_{i} \Gamma, i=1, \cdots, k$. Clearly the $(\mathscr{L}, \Delta)$ cosets in $\mathscr{N}$ which are contained in $\mathscr{R} n_{i} \Gamma$ are the left translates by $n_{i}$ of the $\left(\mathscr{M}_{i}, \Delta\right)$ cosets in $\mathscr{R}_{i} \Gamma$. Let $\mathscr{L}_{i} x \Delta$ and $\mathscr{L}_{i} x^{\prime} \Delta$ be two $\left(\mathscr{N}_{i}, \Delta\right)$ cosets in $\mathscr{R}_{i} \Gamma$. Then $x=r \gamma$ and $x^{\prime}=r^{\prime} \gamma^{\prime}$ with $r, r^{\prime} \in \mathscr{R}_{i}$ and $\gamma, \gamma^{\prime} \in \Gamma$. Suppose $\mathscr{M}_{i} x \Delta=\mathscr{M}_{i} x^{\prime} \Delta$. Then $r^{\prime} \gamma^{\prime}=m r \gamma \delta$ with $m \in$ $\mathscr{N}_{i}, \delta \in \Delta$. Thus $(m r)^{-1} r^{\prime}=\gamma \delta \gamma^{\prime-1}=\delta_{0}$ is in $\mathscr{R}_{i} \cap \Gamma$, and $\boldsymbol{r}^{\prime}=m r \delta_{0}$ and $\gamma^{\prime}=\delta_{0}^{-1} \gamma \delta$. Conversely, if these relations hold, it is easy to see that $\mathscr{A}_{i} x \Delta=\mathscr{A}_{i} x^{\prime} \Delta$. In fact, if only $\gamma^{\prime}=\delta_{0}^{-1} \gamma \delta$, then if we define $r^{\prime}=m r \delta_{0}$ with $m$ arbitrary in $\mathscr{A}_{i}$, we get a representative $x^{\prime}=r^{\prime} \gamma^{\prime}$ for $\mathscr{N}_{i} x \Delta$. Thus $\gamma^{\prime}$ may be arbitrary in $\gamma \Gamma_{i}$, and the $\left(\mathscr{N}_{i}, \Delta\right)$ double cosets in $\mathscr{R}_{i} \Gamma \cap \mathscr{N}$ are in one-to-one correspondence with the $\Gamma_{i}$ cosets in $\Gamma$, and a set of double coset representatives is provided by $\left\{r_{i j} \eta_{i j}\right\}_{j=1}^{l(i)}$ with $\eta_{i j}$ as before and $r_{i j} \in \mathscr{R}_{i}$ such that $r_{i j} \eta_{i j} \in \mathscr{N}$. Finally we conclude that the good $(\mathscr{L}, \Delta)$ cosets in $\mathscr{N}$ such that $\nu$ occurs in $L^{2}(\mathscr{M} \mid \mathscr{M} \cap \operatorname{Ad} n(\Delta))$ are the cosets $\left\{\mathscr{M} x_{i j} \Delta\right\}_{i=1, j=1}^{k l(i)}$, where $n_{i} r_{i j} \eta_{i j}=$ $x_{i j}$.

Put $\mathscr{M}_{i j}=\operatorname{Ad} x_{i j}^{-1} \mathscr{A}=\operatorname{Ad}\left(r_{i j} \eta_{i j}\right)^{-1}\left(\mathscr{C}_{i}\right)=\operatorname{Ad} \eta_{i j}^{-1}\left(\mathscr{M}_{i}\right)$. Let $\tilde{\alpha}_{i j}^{0}: C_{c}\left(\mathscr{N} / \mathscr{M}_{i j} \cap \Delta\right) \rightarrow C_{c}(\mathscr{N} / \Delta)$ be the standard averaging maps. Let $X_{i j}$ be the closure in $L^{2}(\mathscr{N} / \Delta)$ of $\tilde{\alpha}_{i j}^{0}\left(C_{c}\left(\mathscr{N} / \mathscr{M}_{i j} \cap \Delta, \mathrm{Ad}^{*} x_{i j}(\nu)\right)\right)$. Richardson [34] has shown that $L^{2}(\mathscr{N} / \Delta, \sigma) \cong \bigoplus_{i, j} X_{i j}$. (Actually, Richardson concerned himself only with the case when $\nu$ is a linear character on $\mathscr{A}$, and also makes some restrictions on $\mathscr{A}$. However, once the multiplicity formula is known for general $\mathscr{C}$ (still with $\nu$ 
one-dimensional), as it is from [16], then, using the fact that if dim $\nu>1$, we can find $\tilde{\mathscr{C}} \cong \mathscr{M}$, such that $\tilde{\mathscr{M}} \mid \tilde{\mathscr{C}} \cap \Delta$ is compact, and such that $\nu$ is induced from a linear character of $\tilde{\mathscr{H}}$, it is seen that very small modifications in Richardson's arguments give the result as stated.)

The $X_{i j}$ are $\mathscr{N}$-invariant subspaces of $L^{2}(\mathscr{N} / \Delta)$. However, when we make the identification $\mathscr{N} / \Delta \cong \mathscr{N} \Gamma / \Gamma$, the $X_{i j}$ are no longer invariant under $\mathscr{N} \Gamma$. Indeed, the transformation $x \rightarrow \gamma x \gamma^{-1}=$ $\operatorname{Ad} \gamma(x)$ for $x \in \mathscr{N}, \gamma \in \Gamma$ preserves $\Delta$ cosets, and so factors to an action of $\Gamma$ on $\mathscr{N} / \Delta$, and this action is the same as the standard left action of $\Gamma$ on $\mathscr{N} \Gamma / \tau$ under the identification of $\mathscr{N} / \Delta$ with $\mathscr{N} \Gamma / \Gamma$. On the other hand, it is immediate from the formulas defining the averaging maps $\widetilde{\alpha}_{i j}^{0}$ that if $\operatorname{Ad}^{*} \Gamma$ denotes the action of $\Gamma$ on $L^{2}(\mathscr{N} / \Delta)$ dual to the action $\operatorname{Ad} \Gamma$ on $\mathscr{N} / \Delta$, then $\operatorname{Ad}^{*} \Gamma$ permutes the $X_{i j}$ among themselves. The orbits consist of all $X_{i j}$ with arbitrary $j$ and fixed $i$. The isotropy group of $X_{i j}$ is simply $\Gamma_{i}$. Therefore $X_{i}=\bigoplus_{j=1}^{l(i)} X_{i j}$ is $\mathrm{Ad}(\Gamma)$-invariant, and so, looked on as subspace of $L^{2}(\mathscr{N} \Gamma / \Gamma)$, it is $\mathscr{N} \Gamma$-invariant. We thus get the orthogonal decomposition $L^{2}(\mathscr{N} \Gamma / \Gamma, \sigma) \cong \bigoplus_{i=1}^{k} X_{i}$. If then $Y_{i}$ is the subspace of $L^{2}(\mathscr{S} \mid \Gamma, \sigma)$ consisting of functions $f$ such that for almost all $s$, the function $n \gamma \rightarrow f(s n \gamma)$ on $\mathscr{N} \Gamma / \Gamma$ is in $X_{i}$, we see that the $Y_{i}$ 's are $\mathscr{S}$-invariant subspaces of $L^{2}(\mathscr{S} \mid \Gamma, \sigma)$, and we have the orthogonal direct sum decomposition $L^{2}(\mathscr{S} \mid \Gamma, \sigma) \cong \bigoplus_{i=1}^{k} Y_{i}$. This is the decomposition we were after. We will now show that $Y_{i}=$ $\beta_{i}\left(L^{2}\left(\mathscr{S} \mid \mathscr{R}_{i} \cap \Gamma, \nu_{i}\right)\right)$, and this will prove the lemma.

We proceed in two steps. First we note as a simple corollary of the discussion of the last paragraph, that if $X_{i j}$ is interpreted as a space of functions on $\mathscr{N} \Gamma_{i} / \Gamma_{i}$, then it is $\mathscr{N} \Gamma_{i}$-invariant. Therefore we may consider $Z_{i j} \subseteq L^{2}\left(\mathscr{S} / \Gamma_{i}\right)$, defined as the space of $f \in L_{2}\left(\mathscr{S} / \Gamma_{i}\right)$ such that for almost all $s \in \mathscr{S}$, the function $n \gamma \rightarrow f(s n \gamma)$ is in $X_{i j}$. The space $Z_{i j}$ is a well-defined $\mathscr{S}$-invariant subspace of $L^{2}\left(\mathscr{S} \mid \Gamma_{i}\right)$. Define the averaging map $\alpha_{i}: L_{2}\left(\mathscr{S} / \Gamma_{i}\right) \rightarrow L^{2}(\mathscr{S} \mid \Gamma)$ by the obvious formula: $a_{i}(f)(s)=\sum_{j=1}^{l(i)} f\left(s \eta_{i j}\right)$. Then it is clear (again by checking the formulas) that $a_{i}\left(Z_{i j}\right) \subseteq Y_{i}$. I claim that in fact $a_{i}\left(Z_{i j}\right)=Y_{i}$. This may be seen directly as follows. Verify easily that if $f \in Z_{i j}$, then the function $s \rightarrow f\left(s \eta_{i l}\right)$ is in $Z_{i m}$, where $\Gamma_{i} \eta_{i j} \eta_{i l}=\Gamma_{i} \eta_{i m}$. Now pick a fundamental domain $U$ for $\Gamma / \Delta$ in $\mathscr{S} / \mathscr{N}$. That is, $U$ is a reasonably nice set (for example, a $G_{\delta}$ ) which contains exactly one point from each $\Gamma / \Delta$ coset in $\mathscr{S} / \mathscr{N}$. It is an easy thing to do to find $U$, since $\mathscr{S} \mid \mathscr{N}$ is an abelian Lie group. Let $U^{\prime}$ denote the inverse image of $U$ in $\mathscr{S}$, and let $U^{\prime \prime}$ be the projection of $U$ into $\mathscr{S} / \Delta$. Then it is quite obvious that the projection of $U^{\prime \prime}$ into $\mathscr{S} \mid \Gamma$ is in fact a bijection. Similarly, it is obvious that if $U_{i j}^{\prime \prime}=\eta_{i j} U^{\prime \prime}=U^{\prime \prime} \eta_{i j}(\Gamma$ may act on $\mathscr{S} / \Delta$ 
on the right because $\Gamma$ normalizes 4 ), and if $U_{i j}^{\prime \prime \prime}$ is the projection of $U_{i j}^{\prime \prime}$ in $\mathscr{S} / \Gamma_{i}$, then the projection of $U_{i j}^{\prime \prime}$ to $U_{i j}^{\prime \prime \prime}$ is a bijection, if $j \neq m$, then $U_{i j}^{\prime \prime \prime}$ and $U_{i m}^{\prime \prime \prime}$ are disjoint and $\mathscr{S} / \Gamma_{i}=\bigcup_{j=1}^{l(i)} U_{i j}^{\prime \prime \prime}$.

The procedure is now clear, though slightly cumbersome (which is typical of this whole construction). Take $f$ in $Y_{i}$. Pull $f$ back to a function $\tilde{f}$ on $U_{i l}^{\prime \prime \prime}$ by means of the bijection between $U_{i l}^{\prime \prime}$ and $\mathscr{S} / \Gamma$. Then we may write $\widetilde{f}=\sum_{j=1}^{l(i)} \widetilde{f}_{j}$, where $\widetilde{f}_{j} \in Z_{i j}$. Let $h_{j}(x)=\widetilde{f}\left(x \eta_{i j}^{-1}\right)$ for $x \in \mathscr{S} / \Gamma_{i}$. Then $h_{j} \in Z_{i l}$, as one verifies immediately by checking the formulas again. Also $h_{j}$ is zero off $\eta_{i j}^{-1} U_{i l}^{\prime \prime \prime}$. Let $h$ be the function on $\mathscr{S} \mid \Gamma_{i}$ which agrees with $h_{j}$ on $\eta_{i j}^{-1} U_{i l}^{\prime \prime \prime}$. Then it is easy to see that the averaging map $a_{i}$ reverses the above process, and $a_{i}(h)=f$.

For the second step, we consider the averaging map $\alpha_{i}^{0}$ defined at the start of the lemma. By the relations stated there, $\alpha_{i}^{0}$ restricted to any coset of $\mathscr{N}$ is just $\tilde{\alpha}_{1}^{0}$. We know from the nilpotent case that the maps from $L^{2}\left(\mathscr{N} / \mathscr{M}_{i} \cap \Gamma, \nu_{i}\right)$ to $X_{i l}$ derived from $\tilde{\alpha}_{i}^{0}$ is onto. Since $L^{2}\left(\mathscr{S} \mid \mathscr{C}_{i} \cap \Gamma, \nu_{i}\right)$ consists of all $f \in L^{2}$ such that on (almost) any coset of $\mathscr{N}$, the restriction of $f$ is in $L^{2}\left(\mathscr{N} / \mathscr{N}_{i} \cap \Gamma, \nu_{i}\right)$, we conclude that the map from $L^{2}\left(\mathscr{S} \mid \mathscr{M}_{i} \cap \Gamma, \nu_{i}\right)$ to $Z_{i l}$ derived from $\alpha_{i}$ is likewise onto. This finishes the lemma.

Now we may combine the foregoing lemmas to obtain our soughtfor result on multiplicity.

THEOREM 2.1. Let $\mathscr{S}$ be a solvable Lie group, $\mathscr{N}$ its nilradical. Suppose $\mathscr{S} / \mathscr{N}$ is abelian. Let $\Gamma$ be a discrete subgroup of $\mathscr{S}$ such that $\mathscr{S} \mid \Gamma$ is compact. Let $\sigma$ be a representation of $\mathscr{N}$ which is invariant under $\mathrm{Ad}^{*} \mathscr{S}$, and which extends to a representation $\rho$ of $\mathscr{S}$. Let $\mathscr{R} \subseteq \mathscr{S}$ be a subgroup such that $\mathscr{R} \mid \mathscr{R} \cap \Gamma$ is compact. Let $\tau$ be a representation of $\mathscr{R}$ which induces $\rho$. Then there are only a finite number of good $(\mathscr{R}, \Gamma)$ double cosets $\mathscr{R} n \Gamma$ such that $\tau$ occurs in $L^{2}(\mathscr{R} \mid \mathscr{R} \cap \operatorname{Ad} n(\Gamma))$ with positive multiplicity $m_{n}$. The multiplicity of $\rho$ in $L^{2}(\mathscr{S} \mid \Gamma)$ is given by the sum $\Sigma m_{n}$ over these good double cosets.

Proof. With Lemmas 2.1 to 2.4 to work with, the proof is a routine matter. We will therefore do something more, and use this opportunity to reinterpret the spectrum of $L^{2}(\mathscr{S} \mid \Gamma, \sigma)$ in the terms of the discussion preceding Lemma 2.1. We reestablish our notations.

Let $\mathscr{M}=\mathscr{R} \cap \mathscr{N}$. We have seen $\mathscr{M} / \mathscr{M} \cap \Gamma$ is closed. Let $\nu$ be the restriction of $\tau$ to $\mathscr{L}$. Let $\left\{\mathscr{R} n_{i} \Gamma\right\}_{i=1}^{k}$ be the $\operatorname{good}(\mathscr{R}, \Gamma)$ double cosets such that $\nu$ occurs in $L^{2}(\mathscr{M} / \mathscr{M} \cap \operatorname{Ad} n(\Gamma))$. Put $\mathscr{R}_{i}=$ Ad $n_{i}^{-1}(\mathscr{R})$, and $\mathscr{K}_{i}=\operatorname{Ad} n_{i}^{-1}(\mathscr{C})$. Denote by $\nu_{i}$ the representation $\operatorname{Ad}^{*} n_{i}(\nu)$ of $\mathscr{L}_{i}$. Recall that $L^{2}\left(\mathscr{S} \mid \mathscr{R}_{i} \cap \Gamma, \nu_{i}\right)$ is defined as the subspace of functions $f \in L^{2}\left(\mathscr{S} \mid \mathscr{R}_{i} \cap \Gamma\right)$ such that for almost all 
$x \in \mathscr{S}$, the function $m \rightarrow f(x m)$ on $\mathscr{A}_{i}$ transforms to the left under $\mathscr{M}_{i}$ according to $\nu_{i}$.

Let $\tau_{i}$ be the representation $\operatorname{Ad}^{*} n_{i}(\tau)$ on $\mathscr{R}_{i}$. Let $H_{i}^{\prime}=$ $\mathscr{A}_{i} \cdot\left(\mathscr{R}_{i} \cap \Gamma\right)$. Let $\tau_{i}^{\prime}$ be the restriction of $\tau_{i}$ to $H_{i}$. We identify $\mathscr{M}_{i} \mid \mathscr{M}_{i} \cap \Gamma$ and $H_{i} / \mathscr{R}_{i} \cap \Gamma$ in the usual way. Suppose $\nu$ occurs in $L^{2}\left(\mathscr{M}_{i} \mid \mathscr{M}_{i} \cap \Gamma\right)$ with multiplicity $a_{i}$. Then according to the discussion of $L^{2}(\mathscr{S} \mid \Gamma, \sigma)$ preceding Lemma 2.1, we have that under the action of $H_{i}, L^{2}\left(\mathscr{A}_{i} \mid \mathscr{N}_{i} \cap \Gamma, \nu\right)$ breaks up into a sum $\sum_{j} b_{i j} \tau_{i}^{\prime} \otimes \zeta_{i j}^{\prime}$, where the $\zeta_{i j}^{\prime}$ are characters of $H_{i} / \mathscr{A}_{i}$, and $\sum_{j} b_{i j}=a_{i}$.

Again referring to the discussion preceding Lemma 2.1, where the representation of $\mathscr{S}$ on $L^{2}\left(\mathscr{S} / \mathscr{R}_{i} \cap \Gamma, \nu_{i}\right)$ is just $\sum_{j} b_{i j} \operatorname{ind}_{H_{i}}^{\mathscr{S}} \tau_{i}^{\prime} \otimes$ $\zeta_{i j}^{\prime}$. If we induce this in stages, going first up to $\mathscr{R}_{i}$, we get $\sum_{j} b_{i j} \operatorname{ind}_{H_{i}}^{\mathscr{R} i} \tau_{i}^{\prime} \otimes \zeta_{i j}=\sum_{j} \sum_{\phi} b_{i j} \tau_{i} \otimes \zeta_{i j} \otimes \phi . \quad$ Here we have identified $H_{i} / \mathscr{N}_{i}$ with a subgroup of $\mathscr{R}_{i} / \mathscr{M}_{i}$ and $\zeta_{i j}$ is any character of $\mathscr{R}_{i} / \mathscr{L}_{i}$ whose restriction to $H_{i}$ is $\zeta_{i j}^{\prime}$. In the second summation $\phi$ runs over all of $\left(\mathscr{R}_{i} / H_{i}\right)^{\wedge}$. Now since $\mathscr{R} \cdot \mathscr{N}=\mathscr{S}$, we may identify $\left(\mathscr{R}_{i} / H_{i}\right)^{\wedge}$ with $\left(\mathscr{S} / \mathscr{N} H_{i}\right)^{\wedge}$, and $\left(\mathscr{R}_{i} / \mathscr{M}_{i}\right)^{\wedge}$ with $(\mathscr{S} / \mathscr{N})^{\wedge}$. Doing so, it follows immediately that upon passing from $\mathscr{R}_{i}$ to $\mathscr{S}$ we get

$$
L^{2}\left(\mathscr{S} \mid \mathscr{R}_{i} \cap \Gamma, \nu_{i}\right) \cong \sum_{j} \sum_{\alpha} b_{i j} \rho \otimes \zeta_{i j} \otimes \varphi_{\alpha}^{i},
$$

where the $\zeta_{i j}$ 's are now interpreted as characters of $\mathscr{S} / \mathscr{N}$, and $\varphi_{\alpha}^{i}$ runs over $\left(\mathscr{S} \mid \mathcal{N} H_{i}\right)^{\wedge}$ as $\alpha$ varies.

Now that we have written this down, we see that Lemmas 2.1 through 2.4 imply that

$$
L^{2}(\mathscr{S} \mid \Gamma, \sigma) \cong \sum_{i} \sum \sum_{\alpha} b_{i j} \rho \otimes \zeta_{i j} \otimes \phi_{\alpha}^{i} .
$$

By inspecting this formula, we will see the theorem is true. By the discussion preceding Lemma 2.1, we see that $\rho \otimes \zeta_{i j} \otimes \varphi$ is equivalent to $\rho$ if and only if $\zeta_{i j} \rho$ is trivial. Moreover, it is completely obvious that the multiplicity of $\tau$ in $L^{2}\left(\mathscr{R} / \mathscr{R} \cap \operatorname{Ad} n_{i}(\Gamma)\right)$ is the same as the multiplicity of $\tau_{i}$ in $L^{2}\left(\mathscr{R}_{i} / \mathscr{R}_{i} \cap \Gamma\right)$. These three facts, plus formula (12) establish Theorem 2.1.

Let us now go somewhat further, and compare (12) with the decomposition of $L^{2}(\mathscr{S} \mid \Gamma, \sigma)$ given before Lemma 2.1. That decomposition was $L^{2}(\mathscr{S} \mid \Gamma, \sigma) \cong \sum_{i} \sum_{\varphi} a_{i}\left(\rho_{1} \otimes \psi_{i}^{\prime} \otimes \phi\right)$ where $\rho_{1}$ was a fixed representation which did occur in $L^{2}(\mathscr{S} \mid \Gamma, \sigma)$, the $\psi_{i}^{\prime}$ are characters of $\mathscr{S} / \mathscr{N}$ whose restrictions to $\mathscr{N} \Gamma$ are specified, and $\varphi$ is arbitrary in $(\mathscr{S} / \mathscr{N} \Gamma)^{\wedge}$. For ease of comparison, put $\rho_{1}=\rho$. In this decomposition, the $\psi_{i}^{\prime}$ were the most mysterious objects. We had no explanation of what they would be or how they would arise. Our decomposition (12) is more refined and provides a partial explanation of the origin of the $\psi_{i}^{\prime}$. Indeed, in comparing the two, we see we must have 
equations $\psi_{k}=\zeta_{i j} \otimes \varphi_{\alpha}^{i}$. Recall that $\zeta_{i j}$ is a character of $\mathscr{S} / \mathscr{N}$ having specified behavior on $\Gamma_{i} / \Delta$ and $\varphi_{\alpha}^{i}$ is trivial on $\Gamma_{i}$. In particular, we see that, in addition to $\psi_{k}$, every character of $\Gamma$ agreeing with $\psi_{k}$ on $\Gamma_{i}$ will occur among the $\psi_{l}$ 's. In terms of the proof of Lemma 2.4, we see that the set of $\psi_{l}$ 's agreeing with $\psi_{k}$ on $\Gamma_{i}$ arise essentially because $\Gamma_{i}=\left(\mathscr{R}_{i} \cap \Gamma\right) \cdot \Delta$ may be a proper subgroup of $\Gamma$, so there may be several $(\mathscr{L}, \Delta)$ cosets in $\left(\mathscr{R} n_{i} \Gamma\right) \cap \mathscr{N}$, in which case $\Gamma / \Gamma_{i}$ permutes transitively the invariant subspaces $X_{i j}$ of $L^{2}(\mathscr{N} / \Gamma, \sigma)$ described in Lemma 2.4. Thus, we see that if we can predict the $\zeta_{i j}$ 's, we can also find the $\psi_{i}$ 's from the $(\mathscr{R}, \Gamma)$ and $(\mathscr{M}, \Delta)$ double coset structure. Thus, if $\mathscr{R}$ is a proper subgroup of $\mathscr{S}$, we have effected a reduction in the problem of determining the $\psi_{l}$ 's. Indeed, if it should happen that we can choose $\mathscr{R}$ so that $\tau$ is one-dimensional, then of course $\nu$ will also be one-dimensional, and $L^{2}\left(\mathscr{M}_{i} \mid \mathscr{M}_{i} \cap \Gamma\right)$ will always be one-dimensional, and $L^{2}\left(\mathscr{R}_{i} / \mathscr{R}_{i} \cap \Gamma, \nu\right)$ will obviously consist of all extensions of $\nu$ to $\mathscr{R}_{i} / \mathscr{R}_{i} \cap \Gamma$. Any two such extensions differ by a character of $\mathscr{R}_{i} / \mathscr{M}_{i}\left(\mathscr{R}_{i} \cap \Gamma\right)$. Hence, in this case the $\zeta_{i j}$ 's are completely determined in an obvious manner, and the complete structure of $L^{2}(\mathscr{S} / \Gamma, \sigma)$ is quite clear.

In view of the above discussion, and also to determine the usefulness of Theorem 2.1, it is of interest to know from what sorts of groups $\mathscr{R}$ we may expect to induce $\rho$. Usually it is too much to expect that we can choose $\mathscr{R}$ so that the inducing representation $\tau$ on $\mathscr{R}$ is one-dimensional. (Indeed, this is not even true when one allows any $\mathscr{R}$, not just those $\mathscr{R}$ such that $\mathscr{R} / \mathscr{R} \cap \Gamma$ is compact.) However, this question does have a fairly satisfactory answer, obtainable by the now standard techniques of Kirillov theory. The result we want is almost, but unfortunately not quite (due to the rather arbitrary nature of our $\mathscr{S}$ ) a consequence of Proposition $1 \mathrm{~A}$ of [19], and so we give a separate proof. The details of the proof are the same as for Proposition 1A of [19], and it seems that a scheme-theoretic version of Proposition 1A would include the present result. In any case, we point out the obvious strong analogy of this result with Proposition 1.3 of part I.

This is the set-up. We take $\mathscr{S}$ and $\mathscr{T}, \Gamma$ and $\Delta$ as always. We take any $\sigma \in \hat{\mathscr{N}}$ which occurs in $L^{2}(\mathscr{N} / \Delta)$. We do not necessarily assume that $\sigma$ extends to $\mathscr{S}$, or even that it is invariant under the action $\operatorname{Ad}^{*} \mathscr{S}$ of $\mathscr{S}$ on $\hat{\mathscr{N}}$ Let $\mathscr{S}_{0}$ denote the isotropy group of $\sigma$ under $\operatorname{Ad}^{*} \mathscr{S}$. Of course $\mathscr{N} \subseteq \mathscr{S}_{\sigma}$, and $\mathscr{S}_{\sigma} / \mathscr{S}_{\sigma} \cap \Gamma$ is compact.

In our present context, that of Lie groups, we will understand a Heisenberg group to be a two-step nilpotent connected simplyconnected Lie group $\mathscr{C}$, whose center and commutator subgroup coincide, and are one-dimensional. 
Proposition 2.3. We may find a subgroup $\mathscr{R} \cong \mathscr{S}$, and a representation $\nu$ of $\mathscr{C}=\mathscr{R} \cap \mathscr{N}$ such that

(i) $\mathscr{R} \mid \mathscr{R} \cap \Gamma$ and $\mathscr{M} \mid \mathscr{M} \cap \Delta$ are compact.

(ii) $\mathscr{S}_{\sigma}=\mathscr{R} \cdot \mathscr{N}$.

(iii) $\sigma=\operatorname{ind}_{\mathscr{N}}^{\mathscr{N}} \nu$.

(iv) $\nu \in \hat{\mathscr{C}}$ is $\mathrm{Ad}^{*} \mathscr{R}$-invariant.

(v) $\mathscr{L}$ is connected.

If $\mathscr{C}^{\prime} \subseteq \mathscr{C}$ is the connected component of the identity of the kernel of $\nu, \mathscr{L} / \mathscr{C}^{\prime}$ is a Heisenberg group, or isomorphic to $\boldsymbol{R}$. Also $\mathscr{L}^{\prime} / \mathscr{L}^{\prime} \cap \Delta$ is compact.

Proof. Let $N$ be the Lie algebra of $\mathscr{N}$, and let $N^{*}$ be the vector space dual of $N$. The original Kirillov theorem [21] asserts the existence of a surjective map $\alpha: N^{*} \rightarrow \hat{\mathscr{N}}$ such that the inverse image of a point in $\hat{\mathscr{N}}$ is an $\mathrm{Ad}^{*} \mathscr{N}$ orbit in $N^{*}$, and which commutes with the actions $\operatorname{Ad}^{*} \mathscr{S}$ of $\mathscr{S}$ on $N^{*}$ and $\hat{\mathscr{N}}$. Denote by $\mathscr{O}$ the $\mathrm{Ad}^{*} \mathscr{N}$ orbit $\alpha^{-1}(\sigma)$ in $N^{*}$. Clearly then $\mathscr{S}_{\sigma}$ is the subgroup of $s \in \mathscr{S}$ such that $\operatorname{Ad}^{*} s(\mathscr{O})=\mathscr{O}$. Let $\lambda$ be a point of $\mathcal{O}$, and let $\mathscr{R}_{\lambda}$ be the isotropy group of $\lambda$. Since $\mathscr{N}$ acts transitively on $\mathscr{O}$, we have $\mathscr{S}_{\dot{\delta}}=\mathscr{R}_{\lambda} \cdot \mathscr{N}$.

As sketched during the proof of Lemma 2.3, the existence of $\Delta$ in $\mathscr{N}$ endows $\mathscr{N}$ with the structure of unipotent algebraic group over $Q$. Also $N$ has the structure of vector space over $Q$, such that $\log \Delta$, the inverse image in $N$ under the exponential map of $\Delta$, spans the $Q$-rational points of $N$. Thus $N^{*}$ has a rational structure, and the rational points of $N^{*}$ are those whose values on log $\Delta$ are rational. It is known ([24]) that with the usual normalization of $\alpha$, that in order for $\sigma$ to occur in $L^{2}(\mathscr{N} / \Delta), \mathcal{O}$ must contain rational points. Let $\lambda \in \mathcal{O}$ be rational. Then $\lambda$ is rational on $\log \Delta$, so a multiple of $\lambda$ is integral on $\log \Delta$. The property of being integral on log $\Delta$ is clearly invariant by $\operatorname{Ad}^{*} \Gamma$, and so $\operatorname{Ad}^{*} \Gamma(\lambda)$ must be discrete. This implies by the argument we have used so often that $\mathscr{R}_{2} / \mathscr{R}_{2} \cap \Gamma$ is compact.

To produce $\mathscr{R}$, we will produce $\mathscr{C} \subseteq \mathscr{N}$ and a representation $\nu \in \hat{\mathscr{C}}$ such that $\mathscr{C}$ is normalized by $\mathscr{R}_{\lambda}, \nu$ is fixed under $\mathrm{Ad}^{*} \mathscr{R}_{\lambda}$, $\mathscr{M} \mid \mathscr{M} \cap \Delta$ is compact, $\mathscr{R}_{\lambda} \cap \mathscr{N} \subseteq \mathscr{M}$ and $\mathscr{C}$ and $\nu$ satisfy (i), (iii), and (v) of the proposition. Then putting $\mathscr{R}=\mathscr{R}_{\lambda} \cdot \mathscr{M}$, we see this $\mathscr{R}$ will satisfy the proposition.

We will suppose that we cannot find $\mathscr{C}$ such that $\nu$ is onedimensional and proceed to the general case. Suppose we can find subalgebras $M_{i}, i=1,2,3$, of $N$ satisfying the following properties:

(a) the $M_{i}$ are Ad $\mathscr{R}_{\lambda}$-invariant,

(b) the $M_{i}$ are $Q$-rational (e.g., spanned by their Q-rational 
elements);

(c) $M_{3}$ contains "maximal subordinate" subalgebras to $\lambda$, in the sense of Kirillov [21];

(d) $M_{1}$ and $M_{2}$ are ideals in $M_{3}$;

(e) $M_{1}=M_{2} \cap \operatorname{ker} \lambda$ and $M_{1} \neq M_{2}$;

(f) $M_{3} / M_{1}$ is a Heisenberg Lie algebra.

Then let $\mathscr{C}$ be analytic subgroup of $\mathscr{N}$ corresponding to $M_{3}, \mathscr{C}^{\prime}$ the subgroup corresponding to $M_{1}$, and $\nu$ the representation of $\mathscr{C}$ corresponding to the $\mathrm{Ad}^{*} \mathscr{C}$ orbit of $\lambda$ restricted to $M_{3}$. Then our property (a) guarantees $\mathscr{C}$ is normalized by $\mathscr{R}_{\lambda}$, and our choice of $\nu$ will be $\mathrm{Ad}^{*} \mathscr{R}_{\lambda}$-invariant. Property (b) guarantees part of (i) and part of (v) of the proposition. Property (c) guarantees (iii) of the proposition by Kirillov [21] and property $(f)$ guarantees the rest of ( $\mathrm{v}$ ) of the proposition. Thus finding such $M_{i}$ will establish the proposition.

We show the existence of the $M_{i}$ by induction on the dimension of $N$, and there are two cases to consider. Let $Z$ be the intersection of the kernel of $\lambda$ with the center of $N$. The subspace $Z$ is rational, since $\lambda$ is rational and the center of $N$ is rational. Also, $Z$ is $\operatorname{Ad~} \mathscr{R}_{\lambda^{-}}$ invariant. The first case occurs when $Z \neq\{0\}$. Then we consider $N^{\prime}=N / Z$. Clearly the action of Ad $\mathscr{R}_{\lambda}$ factors to $N^{\prime}$ and $Z$ is Ad $\mathscr{R}_{\lambda}$-invariant. Also $\lambda$ factors to $N^{\prime}$ since $Z \subseteq \operatorname{ker} \lambda$. Then if $M_{i}^{\prime}, i=1,2,3$ are subalgebras of $N^{\prime}$ satisfying properties (a) through $(f)$, it is easy to check that if $M_{i}, i=1,2,3$ are the inverse images in $N$ of the $M_{i}^{\prime}$, then the $M_{i}$ also satisfy properties (a) through $(f)$. Hence in this case, by the inductive hypothesis, we are done.

In the second case, $Z=\{0\}$. Then $\mathscr{Z}(N)$, the center of $N$, is one-dimensional, and $\lambda$ is nontrivial on $\mathscr{Z}(N)$. Let $\mathscr{L}^{2}(N)$ be the second center of $N$ - that is, the inverse image in $N$ of $\mathscr{Z}(N / \mathscr{Z}(N))$. Then $\mathscr{Z}^{2}(N)$ is rational. Let $N^{(2)}$ here denote the centralizer of $\mathscr{2}^{(2)}(N)$.

Clearly $\mathscr{Z}^{2}(N)$ and $N^{(2)}$ are invariant by $\operatorname{Ad} \mathscr{R}_{\lambda}$. Also Ad $\left(\mathscr{R}_{\lambda} \cap \mathscr{N}\right)$ must act trivially on $\mathscr{\digamma}^{2}(N)$, since it fixes $\lambda$, so that the Lie algebra of $\mathscr{R}_{\lambda} \cap \mathscr{N}$ is contained in $N^{(2)}$. Let $N^{\prime}=\mathscr{\varkappa}^{2}(N)+N^{(2)}$. Then either $N^{\prime}$ is a proper subalgebra of $N$, or $N$ is already Heisenberg (or one-dimensional, which case we have agreed to ignore). In either case we can clearly find subalgebras $M_{i}^{\prime}, i=1,2,3$ of $N^{\prime}$ which satisfy properties (a) through $(f)$ with respect to the restriction of $\lambda$ to $N^{\prime}$. But since it is easy to check that $\operatorname{dim} N-\operatorname{dim} N^{\prime}=\operatorname{dim} \mathscr{2}\left(\mathscr{Z}^{2}(N)\right) 1-=$ $\operatorname{dim}\left(\mathscr{Z}^{2}(N) \cap N^{(2)}\right)-1$, it follows that property (c), the only property that might fail when the $M_{i}^{\prime}$ are considered as subalgebras of $N$ rather than $N^{\prime}$, persists, so the $M_{i}^{\prime}$ work also for $\lambda$ and $N$. This proves the proposition.

Proposition 2.3 shows how far things can be reduced in the 
general situation but it is of interest also to know conditions which guarantee that we may choose $\nu$ one-dimensional. Perhaps the most satisfactory general class of groups for which we may always choose $\nu$ one-dimensional are those groups $\mathscr{S}$ which are the intersection of the kernels of the rational characters (in the sense of algebraic groups) of the real points of an algebraic group $\mathscr{S}^{\prime}$ over $Q$, obtained by reduction of scalars from a split group $\mathscr{S}^{\prime \prime}$ over some number field. Such groups will come in for special attention in part four.

By Propositions 2.1 and 2.3, we may consider that we have reduced the general multiplicity computation to the special case when the nilradical $\mathscr{N}$ of $\mathscr{S}$ is Heisenberg, and Ad $\mathscr{S}$ acts trivially on $\mathscr{Z}(\mathscr{N})$, the center of $\mathscr{N}$. Indeed, with Theorem 2.1, we have done slightly better than that. We will finish part two by making explicit precisely how much better we have done. Keeping Theorem 2.1 in mind, we will say $\mathscr{S}$ is minimal if

(a) $\mathscr{N}$ is Heisenberg;

(b) Ad $\mathscr{S}$ acts trivially on $\mathscr{Z}(\mathscr{N})$;

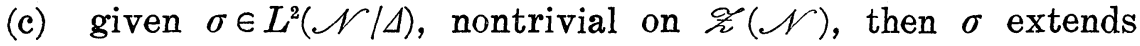
to $\mathscr{S}$; and

(d) there is no proper connected subgroup $\mathscr{C}$ of $\mathscr{N}$ such that $\mathscr{C} \mid \mathscr{M} \cap \Delta$ is compact, $\mathscr{M}$ is normal in $\mathscr{S}$, and $\sigma$ can be induced from $\mathscr{A}$.

Clearly we have reduced to the case of minimal $\mathscr{S}$. Now let us translate the conditions of minimality into conditions on the structure of $\mathscr{S}$, independent of $\sigma$.

Let $\mathscr{S}$ be minimal. Let $\mathscr{Z}(N)$ be the center of the Lie algebra $N$ or $\mathscr{N}$. Let $V=N / \mathscr{Z}(N)$. The action Ad $\mathscr{S}$ of $\mathscr{S}$ on $N$ factors to an action $\operatorname{Ad} \mathscr{S}$ on $V$, and $\mathscr{N}$ acts trivially on $V$, so that Ad $\mathscr{S}$ on $V$ is actually the action of the abelian group $\mathscr{S} / \mathscr{N}$. As is wellknown [19], the commutator on $N$ factors to $V$ and there defines a symplectic form, which we will denote by $\langle$,$\rangle . Since Ad \mathscr{S}$ acts by automorphisms on $N$, and acts trivially on $\mathscr{Z}(N)$, the action Ad $\mathscr{S}$ on $V$ preserves $\langle$,$\rangle . The image in V$ of $\log \Delta$ is a lattice $L$, and $V$ has the structure of vector space over $Q$, with $L$ spanning (over $Q$ ) the $Q$-rational points of $V$. The action of $\operatorname{Ad} \Gamma$ on $V$ of course preserves $L$.

Proposition 2.4. Let $\mathscr{S}$ be minimal. Then

(i) For any Ad $\mathscr{S}$-invariant rational subspace $U$ of $V$, the restriction of $\langle$,$\rangle to U$ is nondegenerate. Equivalently, there are no Ad $\mathscr{S}$-invariant rational subspaces of $V$ which are isotropic with respect to $\langle$,$\rangle .$

(ii) Ad $\mathscr{S}$ acts semisimply on $V$. 
(iii) $\mathscr{S}^{\prime}$ is isomorphic to a semidirect product $\mathscr{S}^{\prime} \cong \mathscr{T} \mathrm{X}_{s} \mathscr{N}$ where $\mathscr{T}$ is abelian and $\mathscr{T} / \mathscr{T} \cap \Gamma$ is compact, and $\mathscr{S}^{\prime}=\mathscr{S} / \operatorname{ker} \sigma$.

We will prove (i), (ii), and (iii) in that order. Recall two simple facts from the representation theory of the Heisenberg groups [24]. If $\mathscr{H} \subseteq \mathscr{N}$ then $\sigma$ can be induced from a representation of $\mathscr{M}$ if and only if $\mathscr{Z}(\mathscr{N}) \subseteq \mathscr{M}$ and $\operatorname{dim} \mathscr{N}-\operatorname{dim} \mathscr{M}=\operatorname{dim} \mathscr{Z}(\mathscr{C})-$ $\operatorname{dim} \mathscr{Z}(\mathscr{N})(=\operatorname{dim} \mathscr{Z}(\mathscr{C})-1)$. This is the same as saying $\mathscr{C}$ is the centralizer in $\mathscr{N}$ of $\mathscr{Z}(\mathscr{C})$. From these facts, we conclude that the condition (d) in the definition of minimality is equivalent to saying there is contained in $\mathscr{N}$ no normal abelian connected subgroup $\mathscr{C}$ of $\mathscr{S}$ such that $\mathscr{M} / \mathscr{M} \cap \Delta$ is compact.

Let $\mathscr{C} \subseteq \mathscr{N}$ contain $\mathscr{Z}(\mathscr{N})$, and let $U$ be the image of the Lie algebra of $M$ in $V$. The following statements are easily checked. The condition that $\mathscr{M} \mid \mathscr{M} \cap \Delta$ be compact is equivalent to the statement that $U / U \cap L$ be compact, that is, that $U$ be rational. The condition that $\mathscr{C}$ be normal becomes the condition that $U$ be $\operatorname{Ad} \mathscr{S}$ invariant. Finally, the condition that $\mathscr{C}$ be abelian is equivalent to the condition that $U$ be isotropic with respect to the form $\langle$,$\rangle .$ These conditions clearly imply the second statement of (i). To see both statements of (i) are equivalent, let $U \subseteq V$ be rational and Ad $\mathscr{S}$-invariant and suppose $\langle$,$\rangle is degenerate on U$. Then if $U_{1}$ is the radical of $\langle$,$\rangle on U$, it is obvious that $U_{1}$ is rational, Ad $\mathscr{S}$ invariant and isotropic with respect to $\langle$,$\rangle and thus cannot exist.$ This finishes (i).

Now we prove (ii). Let $V_{Q}$ be the $Q$-rational points of $V$. Then Ad $\Gamma$ acts on $V_{Q}$ preserving $\langle$,$\rangle . The group of all linear transfor-$ mations of $V_{Q}$ preserving $\langle$,$\rangle is obviously an algebraic group. By$ a standard theorem in algebraic groups [8], given $\gamma \in \Gamma$, we may write $\operatorname{Ad} \gamma=s \cdot u$ where $s$ is a semisimple and $u$ is an unipotent transformation of $V_{Q}$. Moreover $s$ and $u$ both preserve $\langle$,$\rangle and have$ the property that they commute with any transformation the commutes with $\mathrm{Ad} \gamma$. If $\mathrm{Ad} \Gamma$ does not act semisimply, then for some $\gamma, u$ is not the identity. For this $\gamma$, let $Y_{Q}$ be the kernel of $1-u$ and let $Y_{Q}^{\prime}$ be the image of $1-u$. Then $Y_{Q}$ and $Y_{Q}^{\prime}$ are proper nontrivial subspaces of $V_{Q}$ and they have nontrivial intersection $Y_{Q}^{\prime \prime}$ since $1-u$ is nilpotent. Let $Y^{\prime \prime}$ be the real span of $Y_{Q}^{\prime \prime}$. Then $Y^{\prime \prime}$ is clearly rational. It will also be Ad $\mathscr{S}$-invariant, since Ad $\mathscr{S}$ will commute with $1-u$. Moreover $Y^{\prime \prime}$ will be isotropic with respect to $\langle$,$\rangle . For if x, y \in Y^{\prime \prime}$, then $x=(1-u) x^{\prime}$ for $x^{\prime} \in V$. Hence

$$
\begin{aligned}
\langle x, y\rangle & =\left\langle(1-u) x^{\prime}, y\right\rangle=\left\langle x^{\prime}, y\right\rangle-\left\langle u\left(x^{\prime}\right), y\right\rangle \\
& =\left\langle x^{\prime}, y\right\rangle-\left\langle x^{\prime}, u^{-1}(y)\right\rangle=\left\langle x^{\prime},\left(1-u^{-1}\right) y\right\rangle=0
\end{aligned}
$$


since $u(y)=y$. Therefore $Y^{\prime \prime}$ cannot exist by (i). Hence Ad $\Gamma$ acts semisimply on $V_{Q}$. Then, since $\mathscr{S} / \Gamma$ is compact, Ad $\mathscr{S}$ acts semisimply on $V$.

Statements (i) and (ii) of the proposition have essentially been consequences of condition (d) of minimality. Statement (iii) depends on condition (c). Without worries about disconnectedness or rationality, statement (iii) is a very standard result, essentially amounting to the existence of a Cartan subalgebra. In our present situation, the basic argument stays unchanged, except we use the structure of 1 , but details must be followed more carefully.

First we will show that we can write $\mathscr{S}=\mathscr{H} \cdot \mathscr{N}^{\prime}$ with $\mathscr{C}$ and $\mathscr{N}^{\prime} \subseteq \mathscr{N}$ having the following properties:

(a) $\mathscr{N}^{\prime} / \mathscr{N}^{\prime} \cap \Delta$ and $\mathscr{H} \mid \mathscr{H} \cap \Gamma$ are compact;

(b) $\mathscr{N}^{\prime}$ is normal in $\mathscr{S}$;

(c) if $\mathscr{N}^{\prime \prime}=\mathscr{H} \cap \mathscr{N}$, then $\operatorname{Ad}(\Gamma \cap \mathscr{H})$ acts trivially on $\mathscr{N}^{\prime \prime} / \mathscr{Z}(\mathscr{N})$.

To do this, take $\gamma \in \Gamma$ and consider Ad $\gamma$ acting on $V$. Write $V=V_{1} \oplus V_{2}$, where $\operatorname{Ad} \gamma$ is the identity on $V_{1}$ and $\operatorname{Ad} \gamma-1$ is nonsingular on $V_{2}$. This is possible since Ad $\gamma$ is semisimple. Since $\gamma \in \Gamma$, we see $V_{1}$ and $V_{2}$ are rational subspaces of $V$. For $i=1,2$, let $\mathscr{N}_{i}$ denote the subgroups of $\mathscr{N}$ corresponding to the inverse images of $V_{i}$ in $N$. Both $\mathscr{N}_{i}$ are normal in $\mathscr{S}$. Let $\mathscr{H}_{1}$ be the inverse image in $\mathscr{N}$ of the centralizer of the image of $\gamma$ in $\mathscr{S} / \mathscr{N}_{1}$. Then $\mathscr{H}_{1}$ is a closed subgroup of $\mathscr{S}$ and $\mathscr{L}_{1} \cap \mathscr{N}=\mathscr{N}_{1}$ by construction. Also $\mathscr{H}_{1} \mid \mathscr{L}_{1} \cap \Gamma$ is compact by our usual argument, since $\gamma \in \Gamma$. I claim $\mathscr{S}=\mathscr{H}_{1} \cdot \mathscr{H}_{2}$. In fact, if $s \in \mathscr{S}$, then $(s, \gamma)=s \gamma s^{-1} \gamma^{-1} \in \mathscr{N}$, and we may look at $v$, the image of $(s, \gamma)$ in $V$. (We may identify $V$ with $\mathscr{N} / \mathscr{Y}(\mathscr{N})$ by the exponential map, which in this situation is a group isomorphism.) Write $v=v_{1}+v_{2}$ with $v_{i} \in V_{i}$. Then $s \in \mathscr{H}_{1}$ if and only if $v_{2}=0$. Let $n \in \mathscr{N}_{2}$, and let $u$ be the image of $n$ in $V$. Then $(n s, \gamma)=n(s, \gamma) n^{-1}(n, \gamma)$, and the image of $(n s, \gamma)$ in $V$ is $v+(1-\operatorname{Ad} \gamma) u$. Since $(1-\operatorname{Ad} \gamma)$ is invertible on $V_{2}$, we may arrange that $(\operatorname{Ad} \gamma-1) u=v_{2}$, in which case $n s=h \in \mathscr{K}_{1}$. Thus $s=n^{-1} h$ and the claim is established.

If we rename $\mathscr{H}_{1}=\mathscr{S}_{,} \mathscr{N}_{1}=\mathscr{N}$, and $\Gamma \cap \mathscr{H}_{1}=\Gamma$, we may repeat the above reduction, until we arrive at a situation where $\operatorname{Ad} \Gamma$ acts trivially on $V$. We note that minimality is preserved under this reduction (that is $\mathscr{C}_{1}$ must be minimal) so $\mathscr{N}_{1}$ and successive subgroups selected by this process will all be Heisenberg groups, unless they reduced to $\mathscr{Z}(\mathscr{N})$, at which point we are done. Thus we have accomplished the desired decompositions $\mathscr{S}=\mathscr{H} \cdot \mathscr{N}^{\prime}$. If in this decomposition $\mathscr{C} \cap \mathscr{N}=\mathscr{Z}(\mathscr{N})$ or if Ad $\mathscr{C}$ is trivial on

$$
(\mathscr{H} \cap \mathscr{N} / \mathscr{Y}(\mathscr{N})) \text {, }
$$


then we are ready for the final step. Otherwise we must make another reduction. So assume $\mathscr{H} \cap \mathscr{N}$ is Heisenberg, and Ad $\mathscr{H}$ is nontrivial on $(\mathscr{H} \cap \mathscr{N}) / \mathscr{F}(\mathscr{N})$. Rename $\mathscr{H}=\mathscr{S}$, and $\mathscr{N} \cap \mathscr{H}=$ $\mathscr{N}$, and $\Gamma \cap \mathscr{H}=\Gamma$. Then we see our new $\mathscr{S}$ is again minimal, and in addition, $\operatorname{Ad} \Gamma$ acts trivially on $\mathscr{N} / \mathscr{F}(\mathscr{N}) \cong V$.

Let $\mathscr{H}_{1}$ now be the kernel of the action of Ad $\mathscr{S}$ on $V$. Then $\mathscr{H}_{1}$ is normal in $\mathscr{S}$, and $\mathscr{N} \cong \mathscr{H}_{1}$ and $\Gamma \subseteq \mathscr{H}_{1}$, so $\mathscr{H}_{1} \mid \Gamma$ and $\mathscr{S} \mid \mathscr{H}_{1}$ are compact. We take note of another easily proved fact about the Heisenberg groups: any automorphism of $\mathscr{N}$ which acts trivially on $\mathscr{N} / \mathscr{Z}(\mathscr{N})$ is inner. It follows that we may write $\mathscr{H}_{1}=\mathscr{N} \cdot C$, where $C$ denotes the centralizer of $\mathscr{N}$ in $\mathscr{C}_{1}$. Of course $C \cap \mathscr{N}=$ $\mathscr{Z}(\mathscr{N})$. Moreover, since the centralizer of $\mathscr{N}$ is the same as the centralizer of $\Delta=\mathscr{N} \cap \Gamma$, we see that $C$ is compact modulo its intersection with $\Gamma$. It is clear also that $C(\mathscr{N})$ is normal in $\mathscr{S}$.

Now we repeat the first reduction, but with $s \in \mathscr{S}$, not in $\Gamma$. Consider Ad $s$ acting on $V$ and write $V=V_{1} \oplus V_{2}$ where Ad $s$ is the identity on $V_{1}$ and 1-Ad $s$ is nonsingular on $V_{2}$. The $V_{i}$ may not be rational, but this need not concern us. Since they are composed of distinct eigenspaces for Ad $s$, they are orthogonal with respect to $\langle$,$\rangle , so the restriction of \langle$,$\rangle to each of them is nondegenerate.$ Let $\mathscr{N}_{i}$ be the connected subgroups of $\mathscr{N}$ corresponding to the inverse images of the $V_{i}$ in $N$. Let $\mathscr{R}$ be the subgroup which is the inverse image in $\mathscr{S}$ of the centralizer of the image of $s$ in $\mathscr{S} / \mathscr{N}_{1}$. Then by the same argument as above, $\mathscr{S}=\mathscr{R} \cdot \mathscr{N}_{2} \cdot$ I claim $C \subseteq \mathscr{R}$. Indeed, $C$ is normal so $(c, s) \in C$ if $c \in C$. But always $(c, s) \in \mathscr{N}$ since $\mathscr{S} / \mathscr{N}$ is abelian. Hence $(c, s) \in C \cap \mathscr{N}=\mathscr{L}(\mathscr{N})$ so $c$ does indeed centralize $s$ modulo $\mathscr{N}_{1}$. By picking a succession of $s$, we see we may arrive at a decomposition $\mathscr{S}=\mathscr{R}^{\prime} \cdot \mathscr{N}^{\prime \prime}$ where $\mathscr{R}^{\prime}$ and $\mathscr{N}^{\prime \prime} \cong \mathscr{N}$ are as follows. Both $\mathscr{N}^{\prime \prime}$ and $\mathscr{N}^{\prime}=\mathscr{R}^{\prime} \cap \mathscr{N}$ are Heisenberg or equal to $\mathscr{Z}(\mathscr{N})$, and both are normal in $\mathscr{S}$. Moreover $C \subseteq \mathscr{R}^{\prime}$ and Ad $\mathscr{R}^{\prime}$ acts trivially on $\mathscr{N}^{\prime} / \mathscr{Z}(\mathscr{N})$. The same reasoning that produced $C$ now shows that $\mathscr{R}^{\prime}=C^{\prime} \cdot \mathscr{N}^{\prime}$ where $C^{\prime}$ is the centralizer of $\mathscr{N}^{\prime}$ in $\mathscr{R}^{\prime}$. Then it follows that $\mathscr{S}=C^{\prime} \cdot \mathscr{N}$ and $C^{\prime} \cap \mathscr{N}=$ $\mathscr{Z}(\mathscr{N})$. Of course $C \cong C^{\prime}$. On the other hand, $C^{\prime} / C \cong C^{\prime} \cdot \mathscr{N} / C \cdot \mathscr{N} \cong$ $\mathscr{S} \mid \mathscr{C}_{1}$ is compact. Since $\mathscr{\mathscr { C }}{ }_{1} \mid \Gamma$ is compact, we conclude $C^{\prime} \mid C^{\prime} \cap \Gamma$ is compact. Hence if $\mathscr{T}^{\prime}=C^{\prime}$, then $\mathscr{T}^{\prime}$ has the properties that $\mathscr{S}=$ $\mathscr{T}^{\prime} \cdot \mathscr{N}$, and $\mathscr{T}^{\prime} / \mathscr{T}^{\prime} \cap \Gamma$ is compact, and $\mathscr{T}^{\prime} \cap \mathscr{N}=\mathscr{F}(\mathscr{N})$.

To complete the proof of (iii) and finish the proposition we want to find $\mathscr{T} \subseteq \mathscr{T}^{\prime}$ such that $\mathscr{T} / \mathscr{T} \cap \Gamma$ is compact, $\mathscr{T} / \operatorname{ker} \sigma$ is abelian, $\mathscr{T} \cap \mathscr{L}(\mathscr{N}) \leqq \operatorname{ker} \sigma$ and $\mathscr{T}^{\prime}=\mathscr{T} \cdot \mathscr{Z}(\mathscr{N})$. If we show $\mathscr{T}^{\prime} / \operatorname{ker} \sigma$ abelian, then it is quite a standard matter to find such a $\mathscr{T}$. On the other hand, if $\mathscr{T}^{\prime} / \operatorname{ker} \sigma$ is nonabelian, it is impossible, because the commutator group of $\mathscr{T}^{\prime}$ lies inside $\mathscr{Y}(\mathscr{N})$, since $\mathscr{T}^{\prime} / \mathscr{Z}(\mathscr{N}) \cong$ $\mathscr{S} / \mathscr{N}$ is abelian. Hence let us show $\mathscr{T}^{\prime} / \operatorname{ker} \sigma$ is abelian. To do this, 
we finally invoke condition (c) of minimality. Notice that up to this time we have depended entirely on structure theory for Heisenberg groups and (c) has played no part. It is here that it enters. Indeed, condition (c) requires that we be able to extend to $\mathscr{S}$ a representation $\sigma$ of $\mathscr{N}, \sigma$ being nontrivial on $\mathscr{Z}(\mathscr{N})$; that is, the Mackey obstruction of extension of $\sigma$ vanishes. It is not hard to compute this obstruction, and this has been done in [5] and [10], where it has been shown that it essentially is equal to the commutator form from $\left(\mathscr{T}^{\prime} / \mathscr{F}(\mathscr{N})\right) \times\left(\mathscr{T}^{\prime} / \mathscr{F}(\mathscr{N})\right)$ to $\mathscr{Z}(\mathscr{N}) / \operatorname{ker} \sigma$. In particular if the Mackey obstruction vanishes, $\mathscr{T}^{\prime} /$ ker $\sigma$ is abelian. This completes Proposition 2.4.

We have now reduced the problem of computing multiplicities for general $\mathscr{S} / \Gamma$ to the case when $\mathscr{S}$ is of the form specified by Proposition 2.4. In part 3 we will consider the multiplicity problem for such $\mathscr{S}$.

\section{REFERENCES}

1. L. Auslander and J. Brezin, Invariant subspace theory for three-dimensional nilmanifolds, B. A. M. S., 78 (1972), 254-258.

2. J. Brezin, Mackey's little group method and $L^{2}$ of compact homogeneous spaces, in Harmonic Analysis on Homogeneous Spaces, Proc. Symp. in Pure Math., vol. XXVI, Amer. Math. Soc., Providence, Rhode Island, 1973.

3. L. Auslander and B. Kostant, Quantization and representations of solvable Lie groups, Bull. Amer. Math. Soc., 73 (1967), 692-695.

4. - Polarization and unitary representations of solvable Lie groups, Inventiones Math., 14 (1971), 255-354.

5. L. Auslander and C. C. Moore, Unitary representations of solvable Lie groups, Mem. of Amer. Math. Soc., 62 (1966).

6. P. Bernat, Sur les representations unitaires des groupes de Lie resolubles, Ann. de L'E. N. S.. 82 (1965), 37-99.

7. R. Blattner, On induced representations, Amer. J. Math., 83 (1961), 79-98.

8. A. Borel, Linear algebraic groups, Proc. Symp. in Pure Math. vol. IX, Amer. Math. Soc., Providence, Rhode Island, (1966), 3-19.

9. A. Borel and J. Tits, Groups reductifs, Pub. I.H.E.S., 27 (1965), 55-150.

10. J. Brezin, Unitary representation theory for solvable Lie Groups, Mem. of Amer. Math. Soc., 79 (1968).

11. F. Bruhat, Sur les representations induites des groupes de Lie, Bull. de la Soc. Math. de France, 84 (1956), 97-205.

12. C. Curtis and I. Reiner, Representation Theory of Finite Groups and Associative Algebras, Interscience, New York, 1962.

13. J. M. C Fell, The dual space of $C^{*}$ algebras, T. A. M. S., 94 (1960), 365-403.

14. R, Godement, Groupes lineaires algebriques sur un corps parfait, Sem. Bourbaki, $\exp 206$, (1961).

15. E. Hewitt and K. Ross, Abstract Harmonic Analysis, Grundlehren der Math. Wissenschaften, 115, Springer-Verlag, Berlin, 1963.

16. $\mathrm{R}$ Howe, Frobenius reciprocity for unipotent algebraic groups over Q, Amer. J. Math., 93 (1971), 163-172

17. - The Fourier transform on nilpotent locally compact groups, I, Pacific J. Math., 73 (1977), 307-327. 
18. R. Howe, Kirillov theory for compact p-adic groups, Pacific J. Math., 73 (1977), 365-381.

19. - On the character of Weil's representation, Trans. Amer. Math. Soc., 177 (1973), 287-298.

20. H. Jacquet and R. P. Langlands, Automorphic Forms on $G l_{2}$, Springer Lecture Notes 114, Springer-Verlag, New York, (1970).

21. A. A. Kirillov, Unitary representations of nilpotent Lie groups, Uspekhi Matem. Nauk, 106 (1962), 57-110.

22. R. P. Langlands, Dimensions of spaces of automorphic forms, Proc. Symp. in Pure Math., vol. IX, Amer. Math. Soc., Providence, Rhode Island, (1966), 253-257.

23. G. W. Mackey, The Theory of Group Representations, mimeographed notes, U. of Chicago, 1955.

24. C. C. Moore, Decomposition of unitary representations defined by discrete subgroups of nilpotent groups, Ann. of Math., 82 (1965), 146-182.

25. - Plancherel formula for non-unimodular groups, Maryland Conference in Harmonic Analysis 1971, Springer Lecture Notes 266.

26. G. D. Mostow, Factor spaces of solvable groups, Ann. of Math., 60 (1954), 1-27.

27. - Representative functions on discrete groups and solvable arithmetic groups, Amer. J. Math., 92 (1970), 1-31.

28. Pontryagin, Topological Groups.

29. L. Pukanszky, On the characters and Plancherel formula of nilpotent groups, J. Functional Analysis, 1 (1967), 255-280.

30. - Unitary representations of solvable Lie groups, Ann. Sci. Ec. Norm. Sup., 4 (1971), 457-608.

31. - Lecons sur les representations des groupes, Dunod, Paris, (1966).

32. - On the theory of exponential groups, T. A. M. S., 126 (1967), 487-507.

33. - Characters of algebraic solvable groups, J. Functional Analysis, 3 (1969), $435-494$.

34. L. Richardson, Decomposition of the $L^{2}$-space of a general compact nilmanifold, Amer. J. Math., 93 (1971), 173-189.

35. A. Weil, L'integration dans les groupes topologiques et ses applications, Actualites Sci. et Ind. 1145, Hermann et Cie., Paris, 1951.

36. - Sur certains groupes d'operateurs unitaires, Acta Math., 111 (1964), $143-211$.

37. _ Basic Number Theory, Springer-Verlag, New York, 1969.

Received May 21, 1977. Supported in part by National Science Foundation Grant GP-7952X3.

YALE UNIVERSITY

New Haven, CT 06520 



\title{
PACIFIC JOURNAL OF MATHEMATICS
}

\section{EDITORS}

\author{
RICHARD ARENS (Managing Editor) \\ University of California \\ Los Angeles, CA 90024 \\ Charles W. Curtis \\ University of Oregon \\ Eugene, OR 97403 \\ C. C. MOORE \\ University of California \\ Berkeley, CA 94720
}

\author{
J. DUGUNDJI \\ Department of Mathematics \\ University of Southern California \\ Los Angeles, CA 90007
}

R. Finn and J. Milgram

Stanford University

Stanford, CA 94305

\section{ASSOCIATE EDITORS}

E. F. BeCKenBaCH

B. H. NeumanN

F. WOLF

K. YoSHIDA

\section{SUPPORTING INSTITUTIONS}

\author{
UNIVERSITY OF BRITISH COLUMBIA \\ CALIFORNIA INSTITUTE OF TECHNOLOGY \\ UNIVERSITY OF CALIFORNIA \\ MONTANA STATE UNIVERSITY \\ UNIVERSITY OF NEVADA, RENO \\ NEW MEXICO STATE UNIVERSITY \\ OREGON STATE UNIVERSITY \\ UNIVERSITY OF OREGON
}

\author{
OSAKA UNIVERSITY \\ UNIVERSITY OF SOUTHERN CALIFORNIA \\ STANFORD UNIVERSITY \\ UNIVERSITY OF HAWAII \\ UNIVERSITY OF TOKYO \\ UNIVERSITY OF UTAH \\ WASHINGTON STATE UNIVERSITY \\ UNIVERSITY OF WASHINGTON
}

The Supporting Institutions listed above contribute to the cost of publication of this Journal, but they are not owners or publishers and have no responsibility for its content or policies.

Mathematical papers intended for publication in the Pacific Journal of Mathematics should be in typed form or offset-reproduced, (not dittoed), double spaced with large margins. Please do not use built up fractions in the text of the manuscript. However, you may use them in the displayed equations. Underline Greek letters in red, German in green, and script in blue. The first paragraph or two must be capable of being used separately as a synopsis of the entire paper. Items of the bibliography should not be cited there unless absolutely necessary, in which case they must be identified by author and journal, rather than by item number. Manuscripts, in triplicate, may be sent to any one of the editors. Please classify according to the scheme of Math. Reviews, Index to Vol. 39. All other communications should be addressed to the managing editor, or Elaine Barth, University of California, Los Angeles, California, 90024.

The Pacific Journal of Mathematics expects the author's institution to pay page charges, and reserves the right to delay publication for nonpayment of charges in case of financial emergency.

50 reprints to each author are provided free for each article, only if page charges have been substantially paid. Additional copies may be obtained at cost in multiples of 50 .

The Pacific Journal of Mathematics is issued monthly as of January 1966. Regular subscription rate: $\$ 72.00$ a year (6 Vols., 12 issues). Special rate: $\$ 36.00$ a year to individual members of supporting institutions.

Subscriptions, orders for numbers issued in the last three calendar years, and changes of address should be sent to Pacific Journal of Mathematics, 103 Highland Boulevard, Berkeley, California, 94708. Older back numbers obtainable from Kraus Periodicals Co., Route 100, Millwood, NY 10546.

\footnotetext{
PUBLISHED BY PACIFIC JOURNAL OF MATHEMATICS, A NON-PROFIT CORPORATION

Printed at Kokusai Bunken Insatsusha (International Academic Printing Co., Ltd.). 8-8, 3-chome, Takadanobaba, Shinjuku-ku, Tokyo 160, Japan.
} 


\section{Pacific Journal of Mathematics}

\section{Vol. 73, No. $2 \quad$ April, 1977}

Roger Evans Howe, On representations of discrete, finitely generated, torsion-free, nilpotent groups ........................ 281

Roger Evans Howe, The Fourier transform for nilpotent locally compact

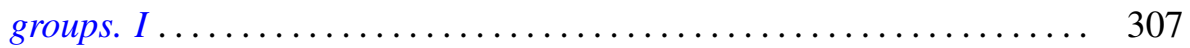

Roger Evans Howe, On a connection between nilpotent groups and

oscillatory integrals associated to singularities............... 329

Roger Evans Howe, Kirillov theory for compact p-adic groups .......... 365

Roger Evans Howe, Topics in harmonic analysis on solvable algebraic

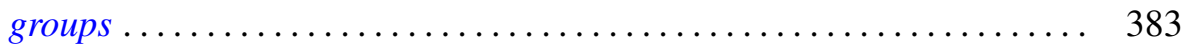

Roger Evans Howe, Tamely ramified supercuspidal representations of

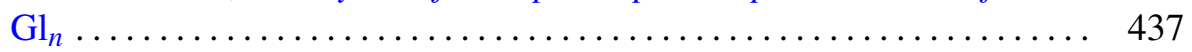

Lawrence Jay Corwin and Roger Evans Howe, Computing characters of tamely ramified $p$-adic division algebras .....................

Roger Evans Howe, Some qualitative results on the representation theory of

$\mathrm{Gl}_{n}$ over a $p$-adic field ............................. 479

Herbert Stanley Bear, Jr., Corrections to: “Ordered Gleason parts”. . . . . . 539

Andreas Blass, Corrections to: "Exact functors and measurable cardinals" .....................................

Robert M. DeVos, Corrections to: "Subsequences and rearrangements of sequences in $\mathrm{FK}$ spaces". 